\title{
A COMPARATIVE STUDY OF FLARING LOOPS IN ACTIVE STARS
}

\author{
D. J. Mullan \\ Bartol Research Institute, University of Delaware, 217 Sharp Laboratory, Newark, DE 19716
}

AND

\author{
M. Mathioudakis, D. S. Bloomfield, and D. J. Christian \\ Department of Physics and Astronomy, Queen's University Belfast, Belfast, BT7 1NN, Northern Ireland, UK \\ Received 2005 September 20; accepted 2006 January 19
}

\begin{abstract}
Dynamo activity in stars of different types is expected to generate magnetic fields with different characteristics. As a result, a differential study of the characteristics of magnetic loops in a broad sample of stars may yield information about dynamo systematics. In the absence of direct imaging, certain physical parameters of a stellar magnetic loop can be extracted if a flare occurs in that loop. In this paper we employ a simple nonhydrodynamic approach introduced by Haisch, to analyze a homogeneous sample of all of the flares we could identify in the EUVE DS database: a total of 134 flares that occurred on 44 stars ranging in spectral type from $\mathrm{F}$ to $\mathrm{M}$ and in luminosity class from V to III. All of the flare light curves that have been used in the present study were obtained by a single instrument (EUVE DS). For each flare, we have applied Haisch's simplified approach (HSA) in order to determine loop length, temperature, electron density, and magnetic field. For each of our target stars, a literature survey has been performed to determine quantitatively the extent to which our results are consistent with independent studies. The results obtained by HSA are found to be well supported by results obtained by other methods. Our survey suggests that, on the main sequence, short loops (with lengths $\leq 0.5 R_{*}$ ) may be found in stars of all classes, while the largest loops (with lengths up to $2 R_{*}$ ) appear to be confined to $\mathrm{M}$ dwarfs. Based on EUVE data, the transition from small to large loops on the main sequence appears to occur between spectral types K2 and M0. We discuss the implications of this result for dynamo theories.
\end{abstract}

Subject headings: stars: flare - stars: magnetic fields

\section{INTRODUCTION}

X-ray images of the Sun indicate that closed loops of magnetic field constitute the "building blocks" of the active corona. These loops exist over a broad range of lengths: there are compact structures, less than $10^{9} \mathrm{~cm}$ long, but there are also structures that are an order of magnitude larger, with lengths of order 0.2 $0.3 R_{\odot}$ (e.g., Golub et al. 1980). The size distribution of magnetic structures may be fitted by a power law, with a spectral index that differs at high and low latitudes (Nakagawa \& Levine 1974).

In contrast to the closed loops that are a bright hallmark of the active Sun, there are also other regions of the Sun where the corona is dark. In such regions, the field is mainly open, and solar wind can escape freely, transporting angular momentum away from the Sun. The relative mixture of closed and open fields has a bearing on the evolution of stellar angular momentum. The fast rotation of certain low-mass stars suggests that such stars do not lose angular momentum as efficiently as the Sun does (Giampapa et al. 1996). This could indicate that closed loops are more prevalent (compared to open field regions) in those stars.

The number of loops in the solar corona rises and falls in the course of the $11 \mathrm{yr}$ cycle. This suggests that loops are the result of oscillatory dynamo operation somewhere in the Sun. There may even be more than one type of dynamo at work. For example, the large-scale solar field may be generated by a dynamo at the interface between the radiative core and the convective envelope, while the small-scale fields may arise from a turbulent dynamo distributed throughout the envelope (Durney et al. 1993).

Whatever the source of the dynamo is, the net effect is that a certain quantity of magnetic flux is supplied to the solar surface per unit time. The flux emerges as bipolar active regions, with loops connecting the two polarities. Depending on the competition between flux supply and flux dissipation (by mass ejections or flares), the (unsigned) magnetic flux on the surface at any time has a certain value $\Phi\left(=\left|\Phi_{+}\right|+\left|\Phi_{-}\right|\right)$that varies by a factor of 4-5 during the cycle (e.g., Schrijver \& Harvey 1994). This flux arranges itself on the surface as a number $N$ of active regions with a surface field strength $|B|$ and a surface area $A$, such that $N|B| A=\Phi$. An active region area $A$ will contain loops with a variety of lengths up to a maximum value $L \approx A^{1 / 2}$. The value of $|B|$ appears to be determined by equipartition between magnetic pressure and photospheric gas pressure (Saar 1990). To the extent that $|B|$ is fixed by equipartition, the activity cycle in $\Phi$ translates mainly into temporal variations in $N$ and/or in $A$, i.e., in $L$.

The unsigned magnetic flux $\Phi$ in various structures in the Sun has been found empirically to be tightly correlated with the X-ray luminosity $L_{\mathrm{X}}$. In fact, the empirical correlation seems to apply just as well to other magnetically active stars (Pevtsov et al. 2003). Moreover, the amount of magnetic flux that is generated by a turbulent dynamo in solar-like stars (Bercik et al. 2005), when combined with the empirical correlation between $L_{\mathrm{X}}$ and $\Phi$, provides a quantitative explanation for the observed lower limits on surface X-ray fluxes in main-sequence stars with spectral types between $\mathrm{F}$ and $\mathrm{M}$. Thus, $L_{\mathrm{X}}$ provides a useful proxy for magnetic flux on stars. In particular, changes in $L_{\mathrm{X}}$ (i.e., flares) provide an opportunity to probe certain aspects of the stellar magnetic field. It is this feature that we exploit in the present paper.

In this paper, because of the connection between dynamo action and $L$, we are interested in deriving information about the lengths of magnetic loops in stars other than the Sun. Now, in the field of 
stellar magnetism, it is certainly a matter of great interest that magnetic fields have recently been detected in some massive stars (Donati et al. 2002). But in the present paper, our interest is restricted to stars in which the internal structure is similar to that in the Sun (with a radiative core and a convective envelope). Moreover, we note that the magnetic dynamo model of Bercik et al. (2005) has been successful in fitting the observed lower envelope of stellar X-ray fluxes only for stars of spectral type F-M. For this reason, in the present study of magnetic structures in stars, we restrict attention to spectral types $\mathrm{F}$ and later. In this way, we hope to learn more about the dynamo processes that occur in solar-like stars. A discussion of dynamo action in stars that are much more massive than the Sun (with a convective core and a radiative envelope) would take us far beyond the limits of the present paper.

We use flares to perform the present study. In this regard, we note that flares on the Sun occur in loops of all sizes, not only in very small loops ("compact flares") but also in larger structures ("two-ribbon flares"). Of course, we cannot hope to determine detailed loop size distributions such as is possible for the Sun (Nakagawa \& Levine 1974). But the study of flares in other stars may provide insight into certain aspects of the loop size distribution, e.g., is the distribution confined to short loops, or does the distribution also extend to long loops?

\subsection{Magnetic Loops on Active Stars}

Active dwarf stars are known to possess magnetic fields on their surfaces, and the field strengths are as large as several kilogauss, i.e., as strong as, or even stronger than, the strongest fields observed on the Sun (e.g., Saar 1990; Johns-Krull \& Valenti 1996). Cyclic behavior has been identified in dozens of stars (Baliunas et al. 1995), suggesting that dynamos not unlike the solar dynamo are also operative in other stars.

Do dynamos in other stars also create loops with a wide range of lengths, or might there be a preferred length for loops in certain stars? Answers to these questions are not known with any confidence. In the case of stars, direct imaging of the loop structures is not yet possible. The use of VLBI does allow the disks of certain favorable objects to be resolved, but just barely (e.g., Pestalozzi et al. 2000).

In the absence of imaging, an indirect approach is called for if we wish to derive the properties of individual loops on other stars.

\section{FLARES: AN OPPORTUNITY TO PROBE UNIMAGED LOOPS}

One such approach becomes available if a flare occurs in a loop. Because the magnetic field constrains the plasma in the loop to move in essentially one dimension, the hydrodynamic processes associated with the deposition of energy in a loop can be modeled numerically with some confidence. A summary of hydrodynamical modeling (HM) of loops in the corona of the Sun can be found in Aschwanden (2004, p. 156), where no less than 18 different HM studies of coronal loops are discussed.

For the case of stars, the release of flare energy in a loop, including the possibility of sustained heating during the decay phase, has also been the subject of HM (e.g., Reale et al. 1997; Favata et al. 2001). Given a flare light curve, this approach allows one to estimate loop lengths and local densities and temperatures.

However, the computational effort in HM is high, and there are a number of poorly known adjustable parameters. This is especially true of the energy deposition function. There are also uncertainties about coronal abundances: the latter have a significant effect on the bound-bound contributions to the radiative cool- ing function near the base of the loop. In view of this, it would be helpful to have a simplified approach to deriving loop parameters in stars, especially if one has a large sample to analyze.

\subsection{Haisch's Approach}

Haisch (1983) suggested one such approach, based on quasistatic radiative and conductive cooling during the earliest phases of flare decay. In the earliest phases of decay, the temperature is high enough (typically $\geq 10 \mathrm{MK}$ ) that radiative losses are dominated by bremsstrahlung emission: in this case, abundance uncertainties are relatively unimportant. Since 1983, other investigators have also used various versions of quasi-static cooling models in order to interpret flare light curves (e.g., van den Oord \& Mewe 1989; Pallavicini et al.1990; Tsuboi et al. 2000). In what follows, for historical reasons, we refer to such approaches generically as "Haisch's simplified approach" (HSA).

Briefly, given an estimate of two measured quantities, the emission measure (EM) in the flare and the decay timescale of the flare $\tau_{d}$, HSA leads to the following expressions for temperature, density, and loop length:

$$
\begin{gathered}
T(\mathrm{~K})=4 \times 10^{-5}(\mathrm{EM})^{0.25} \tau_{d}^{-0.25} \\
N_{e}\left(\mathrm{~cm}^{-3}\right)=10^{9}(\mathrm{EM})^{0.125} \tau_{d}^{-1.125} \\
L(\mathrm{~cm})=5 \times 10^{-6}(\mathrm{EM})^{0.25} \tau_{d}^{0.75}
\end{gathered}
$$

The attractiveness of these formulae is obvious: even for a star where no imaging is possible, we can, with the help of two measured quantities $\left(\mathrm{EM}, \tau_{d}\right)$, extract information about certain physical parameters on a flaring loop.

As an auxiliary quantity, a minimum magnetic field strength is estimated from the condition

$$
B^{2} / 8 \pi \geq 2 N_{e} k_{\mathrm{B}} T
$$

where $k_{\mathrm{B}}$ is Boltzmann's constant.

In the years since HSA first appeared, other approaches, based on different physical principles, have also been proposed that allow one to extract loop parameters from unimaged observations, using compact formulae. One of these, by Shibata \& Yokoyama $(1999,2002)$, is based on a balance between heating due to magnetic reconnection (MR) and chromospheric evaporation (CE). We refer to this for brevity as the MR/CE model. In agreement with HSA, Shibata \& Yokoyama (1999) stress that "the loop length is a key parameter for determining the temperature and EM of solar and stellar flares." It is this sensitivity to loop length that allows both HSA and MR/CE to overcome the lack of imaging. In order to appreciate how the $\mathrm{MR} / \mathrm{CE}$ model differs from HSA, we note that, in order to extract a value of $T, N_{e}$, or $L$ from MR/CE, one must assume a value for $B$ in the flare. Given a $B$-value, the MR/CE model reproduces remarkably well the empirical relationship between EM and $T$. As in equation (1), the value one computes for $T$ in the MR/CE model is found to be rather insensitive to the value of EM. However, HSA relies on two measured quantities (EM and $\tau_{d}$ ), whereas the reconnection model relies on one measured quantity (EM) and one estimated quantity $(B)$. In essence, rather than using a measured timescale, the timescale in the MR/CE model is assumed to be the MHD crossing time $L / v_{\mathrm{A}}$, where $v_{\mathrm{A}}$ is the Alfvén speed. How good is the assumption that the value of $B$ in a stellar flare is reliably known? Shibata \& Yokoyama $(1999,2002)$ plot their results with $B$-values ranging from 15 to $150 \mathrm{G}$, with $B=50 \mathrm{G}$ providing the best fit to the data. But in the results presented 
below, the $B$-values that we derive for flaring loops in our sample of stars span a much wider range than that considered by Shibata \& Yokoyama (1999, 2002): our range goes from 30 to $1800 \mathrm{G}$. Since the predicted value of $\mathrm{EM}$ in MR/CE is quite sensitive to the choice of $B\left(\mathrm{EM} \sim B^{-5}\right)$, our factor of 60 range of $B$-values in flaring loops would lead to predictions of EM values that span a very broad swath of the EM- $T$ diagram.

In the present work we restrict our attention to HSA, where we rely on two measured quantities.

\subsection{HSA Reliability: A First Look}

A question that is central to the present study is the following: how reliable are the estimates of loop parameters obtained by HSA? To address this question, we have undertaken an extensive comparison between the loop parameters obtained by HSA and loop parameters obtained in other ways. We discuss this comparison in detail in $\S 7$. Here we make some preliminary remarks about HSA reliability.

In the context of the analysis that we undertake in the present paper, one aspect of equations (1)-(3) is of prime importance: the numerical values we derive for the various parameters are quite insensitive to the value of the EM. Even if our estimates of EM are in error by factors as large as 2 (which is quite possible; see $\S 4.3$ ), our estimates of $N_{e}$ will be in error by less than $10 \%$, while our estimates of $T$ and $L$ will be in error by less than $20 \%$. This is a significant advantage of our analysis.

Schmitt et al. (1987a), in a study of solar flare X-rays reflected off the Earth, concluded that HSA is "basically sound" as a method for obtaining densities and loop lengths in stellar flares.

Moreover, Covino et al. (2001) have applied one version of HSA to a number of X-ray flares on stars: the focus in their study was on estimating the loop length $L$. (This is the parameter that is of most interest to us in the present study.) Covino et al. (2001) report that in almost all of the flares they analyze, there is a good overall correspondence between $L$-values derived by HM and those obtained by HSA. This is a valuable conclusion in the context of the present work.

However, in some cases, Covino et al. (2001) found that HM yielded $L$-values that were clearly smaller (by factors of $\sim 2$ ) than HSA gave. But there are also two cases where HM yields $L$-values that clearly exceed the HSA lengths by factors of 2-3. A previous example of an HM loop length exceeding the HSA length by a factor of $\sim 3$ was reported by Reale et al. (1988) for a flare on Prox Cen, and three flares in which the HM lengths are less than the HSA lengths by factors of $\sim 2, \sim 2$, and $\sim 5$, respectively, are reported by Favata et al. (2001).

An important feature of the work by Covino et al. (2001) is that they evaluated the statistical significance of the discrepancies between loop lengths evaluated by HM and those evaluated by HSA. This led to the conclusion that such discrepancies, although at first sight relatively large (factors of $\sim 2$ ), actually correspond, in the context of data uncertainties, to $1 \sigma$ effects. Covino et al. (2001), referring to this as a "surprising coincidence," discuss possible reasons why two such different methods can yield results that are within $1 \sigma$ of each other, at least as far as $L$ is concerned.

Overall, the above work suggests that estimates of $L$-values by HSA, although reliable in a general sense, may be subject to errors that are as large as a factor of 2 either too large or too small. We return to this topic in $\S 8.2$.

\subsection{Aim of the Present Work}

In the present work we would like to determine whether or not the loop parameters that emerge from HSA find corrobo- rative support from other approaches. Since we are interested in dynamos, our primary interest is in estimates of $L$ and $B$. However, since the model also yields $N_{e}$ and $T$ estimates, we evaluate the reliability of those parameters as well.

As regards the work of Covino et al. (2001), we note that the flare data that they analyzed were obtained by six different spacecraft (Einstein, EXOSAT, Ginga, ROSAT, ASCA, and BeppoSAX). Since different $X$-ray detectors have different spectral responses to the flaring plasma in any given flare (especially as the plasma cools), it is not clear to what extent the $L$-values derived by Covino et al. (2001) may be affected by variations in instrumental characteristics.

In order to avoid this complication, we have confined our attention in the present study to the analysis of a data set that is strictly homogeneous: all of the data were obtained by a single instrument on the Extreme Ultraviolet Explorer (EUVE). Moreover, the flares analyzed by Covino et al. (2001) were confined to two K stars and two M stars. Here we expand the range of spectral types to include stars of types F, G, K, and M; for brevity, we refer to these as "solarlike stars."

As a result of our survey, we have identified 44 F-M stars that underwent at least one flare. Applying HSA, we seek to obtain the loop parameters $L, N_{e}, T$, and $B$ for 134 individual flares on these 44 stars.

In our survey of the EUVE Deep Survey (DS) database, we have striven to identify as many as possible of the flares that are detectable in the EUVE DS on solar-like stars. We recognize that some small events may have escaped our attention. Nevertheless, we believe that our sample of EUVE DS flares on solar-like stars does not suffer greatly from effects of incompleteness.

Because of the strictly homogeneous nature of the data, we consider that our approach should yield results that are more reliable in a differential (as opposed to absolute) sense. Specifically, we regard our results as being primarily valuable in enabling the comparison of loop properties among stars of different spectral types. However, when the opportunity arises, we also evaluate the absolute reliability of this approach by comparing our results with loop parameters that have been derived using other analyses of an independent nature.

\section{OBSERVATIONS}

All of the data analyzed here were obtained by EUVE. The instrument that gathered the data was the Deep Survey/Spectrometer Instrument (DS/S). The DS/S consists of a grazing incidence Wolter-Schwarzschild type II telescope that performs two functions, one spectroscopy and the other broadband photometry (for a schematic diagram see Sirk et al. 1997).

For reasons that were outlined in $\S 1$, we have restricted our attention in the present study to target stars of spectral class F or later. Some of the data sets were rejected for one or more of the following reasons: (1) the data were affected by the dead spot of the EUVE detector; (2) no source was detectable in the image; (3) a source was visible, but no flares were observed; (4) only an incomplete flare light curve was obtained; (5) the light curve was too complicated to be susceptible to straightforward analysis.

The data sets that we eventually used for our study, including dates and exposure times, are listed in Table 1. For the RS CVn systems in Table 1, many of the light curves have previously been studied by Osten \& Brown (1999); however, although those authors reported decay timescales for many flares, they did not use the HSA to interpret the decay times. The stars in Table 1 are arranged in order of the spectral type of the active component. Spectral types were taken mainly from the SIMBAD database, supplemented where appropriate by information from papers 
TABLE 1

EUVE Data Used in Analysis

\begin{tabular}{|c|c|c|c|c|c|c|c|}
\hline Star & Date & $\begin{array}{l}\text { Exposure } \\
\quad(\mathrm{ks})\end{array}$ & Spectral Type & $R / R_{\odot}$ & $\begin{array}{c}R / R_{\odot} \\
\text { Reference }\end{array}$ & $B-V$ & $V-I$ \\
\hline \multirow[t]{2}{*}{ 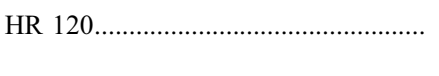 } & 1995 Jul 05 & 57 & $\mathrm{~F} 2 \mathrm{~V}$ & 3.0 & 1 & 0.37 & 0.43 \\
\hline & 1995 Aug 09 & 182 & $\mathrm{~F} 2 \mathrm{~V}$ & 3.0 & 1 & 0.37 & 0.43 \\
\hline 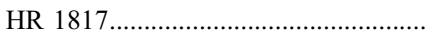 & 1995 Oct 23 & 178 & $\mathrm{~F} 8 / 9$ & 1.65 & 1 & 0.55 & 0.63 \\
\hline$\sigma^{2} \mathrm{CrB} \ldots \ldots$ & 1994 Feb 16 & 385 & $\mathrm{~F} 6 \mathrm{~V}+\mathrm{G} 0 \mathrm{~V}$ & 1.21 & 2 & 0.60 & 0.67 \\
\hline 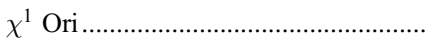 & 1993 Jan 26 & 383 & G1 V & 0.96 & 3 & 0.59 & 0.66 \\
\hline 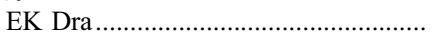 & 1995 Dec 06 & 156 & G1.5 V & 0.85 & 4 & 0.63 & 0.69 \\
\hline \multirow{2}{*}{ 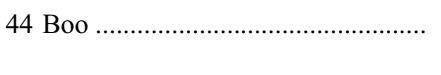 } & 1994 May 02 & 139 & $\mathrm{G} 2 \mathrm{~V}+\mathrm{G}$ & 0.87 & 5 & 0.65 & 0.71 \\
\hline & 1996 Feb 26 & 100 & $\mathrm{G} 2 \mathrm{~V}+\mathrm{G}$ & 0.87 & 5 & 0.65 & 0.71 \\
\hline \multirow{3}{*}{ DK UMa } & 1997 May 14 & 226 & G4 III/IV & 3.5 & 6 & 0.78 & 0.83 \\
\hline & 1994 Oct 13 & 158 & G5 V & 0.93 & 7 & 0.68 & 0.73 \\
\hline & 1995 Oct 06 & 168 & G5 V & 0.93 & 7 & 0.68 & 0.73 \\
\hline \multirow{5}{*}{ 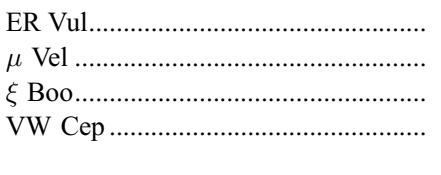 } & 1995 Sep 20 & 630 & G5 V & 1.45 & 8 & 0.61 & 0.68 \\
\hline & 1998 Mar 18 & 1016 & G5 III + F V & 13.0 & 4 & 0.90 & 0.91 \\
\hline & 1997 Apr 20 & 939 & $\mathrm{G} 8 \mathrm{~V}+\mathrm{K} 4 \mathrm{~V}$ & 0.89 & 4 & 0.72 & 0.82 \\
\hline & 1995 Jan 29 & 432 & K0 V (var.) & 0.93 & 9 & 0.86 & 0.87 \\
\hline & 1998 Oct 28 & 1227 & K0 V (var.) & 0.93 & 9 & 0.86 & 0.87 \\
\hline 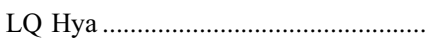 & 1993 Dec 10 & 150 & $\mathrm{~K} 0 \mathrm{Ve}$ & 0.93 & 10 & 0.93 & 1.04 \\
\hline BH CVn & 1996 Feb 12 & 189 & $\mathrm{~F} 2 \mathrm{IV}+\mathrm{K} 0 \mathrm{IV}$ & 3.4 & 11 & 0.93 & 1.00: \\
\hline \multirow{3}{*}{ 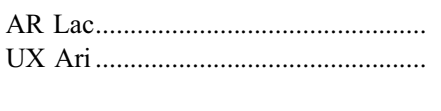 } & 2000 Sep 15 & 225 & G2 IV + K0 IV & 3.1 & 2 & 0.76 & 0.80 \\
\hline & 1995 Nov 07 & 258 & G5 V + K0 IV & 5.78 & 12 & 0.88 & 0.89 \\
\hline & 1995 Nov 19 & 510 & G5 V + K0 IV & 5.78 & 12 & 0.88 & 0.89 \\
\hline \multirow{6}{*}{ 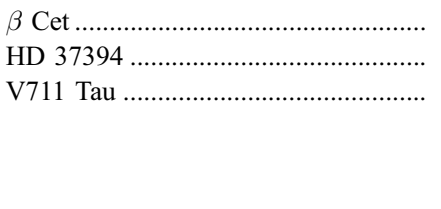 } & 1994 Sep 30 & 305 & K0 III & 15.1 & 2 & 1.02 & 1.00 \\
\hline & 2000 Dec 25 & 380 & $\mathrm{~K} 1 \mathrm{~V}$ & 0.9 & 13 & 0.84 & 0.88 \\
\hline & 1993 Sep 16 & 450 & G5 IV + K1 IV & 3.9 & 12 & 0.99 & 1.00: \\
\hline & 1994 Aug 24 & 348 & G5 IV + K1 IV & 3.9 & 12 & 0.99 & 1.00: \\
\hline & 1996 Sep 01 & 835 & G5 IV + K1 IV & 3.9 & 12 & 0.99 & 1.00: \\
\hline & 1998 Sep 03 & 750 & G5 IV + K1 IV & 3.9 & 12 & 0.99 & 1.00: \\
\hline \multirow{3}{*}{ AR Psc } & 1997 Aug 26 & 131 & G7+K1 IV & 3.4 & 11 & 0.99 & 1.00: \\
\hline & 1998 Dec 10 & 978 & $\mathrm{~K} 1 \mathrm{III}+?$ & 9.3 & 12 & 1.12 & 1.12 \\
\hline & 1999 Dec 30 & 435 & $\mathrm{~K} 1 \mathrm{III}+?$ & 9.3 & 12 & 1.12 & 1.12 \\
\hline \multirow{2}{*}{ 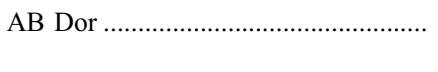 } & 1993 Nov 04 & 588 & K1 IIIp & 1.0 & 14 & 0.83 & 0.94 \\
\hline & 1994 Nov 12 & 102 & K1 IIIp & 1.0 & 14 & 0.83 & 0.94 \\
\hline \multirow{3}{*}{ Gl 117} & 1994 Dec 02 & 192 & $\mathrm{~K} 2 \mathrm{~V}$ & 0.85 & 14 & 0.86 & 1.04 \\
\hline & 1993 Oct 22 & 178 & $\mathrm{~K} 2 \mathrm{~V}$ & 0.75 & 2 & 0.88 & 0.94 \\
\hline & 1995 Sep 05 & 621 & $\mathrm{~K} 2 \mathrm{~V}$ & 0.75 & 2 & 0.88 & 0.94 \\
\hline \multirow{4}{*}{ 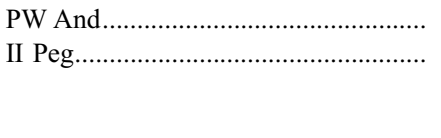 } & 1995 Sep 26 & 705 & $\mathrm{~K} 2 \mathrm{~V}$ & 0.8 & 3 & 1.04 & 0.94: \\
\hline & 1995 Aug 08 & 308 & $\mathrm{~K} 2 \mathrm{IV}+\mathrm{M} 0-\mathrm{M} 3$ & 3.4 & 15 & 1.04 & 2.00: \\
\hline & 1998 Sep 11 & 113 & $\mathrm{~K} 2 \mathrm{IV}+\mathrm{M} 0-\mathrm{M} 3$ & 3.4 & 15 & 1.04 & 2.00: \\
\hline & 1999 Oct 21 & 175 & $\mathrm{~K} 2 \mathrm{IV}+\mathrm{M} 0-\mathrm{M} 3$ & 3.4 & 15 & 1.04 & 2.00: \\
\hline \multirow{5}{*}{ 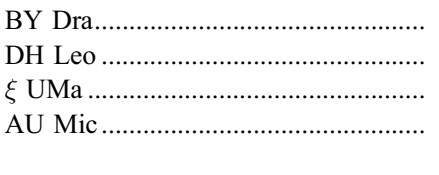 } & 1997 Sep 22 & 575 & $\mathrm{~K} 4 \mathrm{~V}+\mathrm{K} 7 \mathrm{~V}$ & 1.3 & 2 & 1.2 & 1.80 \\
\hline & $1995 \mathrm{Feb} 12$ & 678 & $\mathrm{~K} 0 \mathrm{~V}+\mathrm{M} 0 \mathrm{~V}(?)$ & 0.5 & 16 & 1.4 & 2.10: \\
\hline & 1997 May 14 & 1510 & G5 V + M0 V(?) & 0.5 & 16 & 1.4 & 2.10: \\
\hline & 1992 Jul 14 & 71 & Mo Ve & 0.56 & 17 & 1.47 & 2.14 \\
\hline & 1996 Jun 12 & 67 & M0 Ve & 0.56 & 17 & 1.47 & 2.14 \\
\hline CC Eri & 1995 Sep 13 & 138 & M0 Vp & 0.81 & 18 & 1.39 & 1.87 \\
\hline 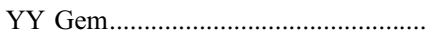 & 1995 Feb 02 & 890 & $\mathrm{M} 1 \mathrm{Ve}+\mathrm{M} 1 \mathrm{Ve}$ & 0.62 & 2 & 1.49 & 2.58 \\
\hline 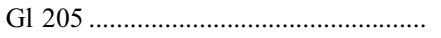 & 1997 Jan 13 & 785 & M1.5 V & 0.70 & 19 & 1.47 & 2.08 \\
\hline 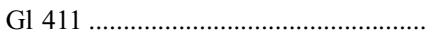 & 1995 Mar 22 & 565 & M2 V & 0.47 & 18 & 1.50 & 2.11 \\
\hline 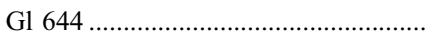 & 1994 Jul 30 & 657 & M3 V & 0.5 & 4 & 1.55 & 2.71 \\
\hline 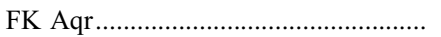 & 1994 Sep 11 & 370 & $\mathrm{M} 2 \mathrm{Ve}+\mathrm{M} 3 \mathrm{Ve}$ & 0.5 & 2 & 1.50 & 2.21 \\
\hline & 1997 Oct 09 & 660 & $\mathrm{M} 2 \mathrm{Ve}+\mathrm{M} 3 \mathrm{Ve}$ & 0.5 & 2 & 1.50 & 2.21 \\
\hline 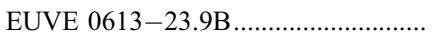 & 2000 Oct 22 & 119 & $\mathrm{M} 3.5 \mathrm{Ve}$ & 0.35 (est.) & & 1.55 & 2.51 \\
\hline AD Leo & 1993 Mar 01 & 235 & M3.5 V & 0.35 (est.) & & 1.54 & 2.51 \\
\hline & 1996 May 03 & 291 & M3.5 V & 0.35 & & 1.54 & 2.51 \\
\hline & 1999 Apr 05 & 415 & M3.5 V & 0.35 & & 1.54 & 2.51 \\
\hline & 1999 Apr 09 & 415 & M3.5 V & 0.35 & & 1.54 & 2.51 \\
\hline & 1999 Apr 17 & 624 & M3.5 V & 0.35 & & 1.54 & 2.51 \\
\hline & 2000 Mar 09 & 294 & M3.5 V & 0.35 & & 1.54 & 2.51 \\
\hline 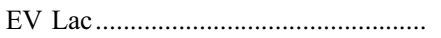 & 1993 Sep 09 & 355 & M3.5 V & 0.35 (est.) & & 1.36 & 2.79 \\
\hline 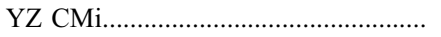 & 1994 Dec 21 & 84 & M4.5 Ve & 0.30 & 17 & 1.60 & 2.95 \\
\hline EQ Peg & 1993 Aug 29 & 40 & M5 Ve & 0.19 & 20 & 1.71 & 2.99 \\
\hline
\end{tabular}


TABLE 1-Continued

\begin{tabular}{|c|c|c|c|c|c|c|c|}
\hline Star & Date & $\begin{array}{l}\text { Exposure } \\
(\mathrm{ks})\end{array}$ & Spectral Type & $R / R_{\odot}$ & $\begin{array}{c}R / R_{\odot} \\
\text { Reference }\end{array}$ & $B-V$ & $V-I$ \\
\hline EQ Peg ........................ & 1996 Oct 02 & 71 & M5 Ve & 0.19 & 20 & 1.71 & 2.99 \\
\hline Prox Cen ........................ & 1993 May 21 & 77 & M5.5 Ve & 0.145 & 19 & 1.81 & 3.62 \\
\hline UV Cet........................ & 1996 Aug 11 & 229 & M5.5 Ve & 0.15 & 18 & 1.85 & 3.69 \\
\hline \multirow[t]{2}{*}{ 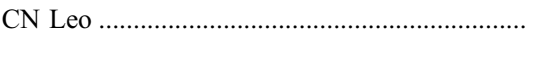 } & 1994 Dec 16 & 61 & M6 Ve & 0.16 & 21 & 2.00 & 4.06 \\
\hline & 1995 Jan 24 & 181 & M6 Ve & 0.16 & 21 & 2.00 & 4.06 \\
\hline
\end{tabular}

References.-(1) Mullan \& Mathioudakis 2000; (2) Sanz-Forcada et al. 2003; (3) Lopez-Santiago et al. 2003; (4) Pasinetti Fracassini et al. 2001; (5) Gondoin 2004b; (6) Ness et al. 2004; (7) Landi et al. 1997; (8) Duemmler et al. 2003; (9) Hill 1989; (10) Donati 1999; (11) estimated from spectral type; (12) Sanz-Forcada et al. 2002; (13) Perrin \& Karoji 1987; (14) Ambruster et al. 2003; (15) Berdyugina et al. 1998; (16) see $\S \S 6.1 .25$ and 6.1.26; (17) Lacy 1977; (18) White et al. 1989; (19) Segransan et al. 2003; (20) Benz et al. 1995; (21) Leggett et al. 2000.

that deal specifically with the system. Radii are taken from a variety of sources, listed at the end of Table 1 . Also included in Table 1 are two colors $(B-V, V-I)$ for each active star. The colors are taken mainly from the online Hipparcos catalog. However, in cases of binaries, where the active component does not dominate in the combined light, we have used estimates of colors based on spectral type. (These estimates are indicated by a colon following the $V-I$ color.) In the case of flare stars for which Hipparcos data are not available (CN Leo, UV Cet, AD Leo, YY Gem), we have obtained the colors from Gershberg (2002).

Properties of the flares that were selected for analysis are given in Tables 2 and 3. The two F stars that appear at the top of the list have already been the subject of a brief version of flare analysis by HSA in an earlier paper (Mullan \& Mathioudakis 2000).

\section{ANALYSIS AND RESULTS}

In this section the processes used in reducing the obtained data sets are presented.

\subsection{DS Analysis}

We obtained our EUVE DS observations from the Multimission Archive at Space Telescope (MAST). The DS data were reduced with the EUVE IRAF packages. DS event lists for each individual source from the archive were converted into quick position oriented event (QPOE) files. QPOE files are essentially a photon list remapped to the position of the source on the sky and contain all the necessary timing information for deriving count rates and light curves. Count rates were determined using appropriate source and background apertures applied to the DS QPOE files. Exposure times for "good" data (spacecraft night time data with no Earth blockage and periods spent passing through the SAA removed) were corrected for telescope vignetting, instrument dead times, and dead times in the spacecraft telemetry (called Primbsching) using the IRAF effexp routine in the euv package. Further details about the EUVE light-curve analysis are given in Christian et al. (1999).

\subsection{Light Curves and $\tau_{d}$}

Initially light curves were plotted using bin sizes of $5000 \mathrm{~s}$, approximately the duration of one 90 minute orbit of the satellite, to establish if any flares were noticeable within the duration of the observation. Better time resolution was achieved for the analysis of the flare curves where bin sizes of typically 200 or 500 s were applied. However, for some source stars with very high count rates these bin durations were able to be reduced to $100 \mathrm{~s}$ and on occasion even as short as $50 \mathrm{~s}$.

From these light curves, three measurable parameters can be found: the mean quiescent source count rate $q$, the flare peak count rate $p_{f}$ at time $t_{f}$, and the flare decay time. The values of $q$ and $p_{f}$ were recorded for each data set and can be found in Tables 2 and 3 .

In order to evaluate $\tau_{d}$, the $e$-folding time of the flare, we first compute the excess of the flare peak about the background: $p_{f}-q$. Dividing this by $e$ and adding on $q$, we obtain the count rate $e_{f}=q+\left(p_{f}-q\right) / e$ that is expected at the "end" of the flare, i.e., by the time $\tau_{d}$ has elapsed since flare peak.

Accurate peak count rates and time bin information were determined from inspection of the light-curve data files. Interpolation of the flare decay from the peak point of the flare down to the level where the count rate is $e_{f}$ yielded the "end time" $t_{e}$ of the flare. From this, the $e$-folding time was obtained as $\tau_{d}=t_{e}-t_{f}$. In certain cases, the data during flare decay were cut short by spacecraft occultation before the count rate had fallen to $e_{f}$. In that case, when the EUVE orbit next allowed data to become available (at time $t_{s}$ ), if the count rate $c_{s}$ is only slightly below $e_{f}$, we used the interval $t_{s}-t_{f}$ as a reasonable upper limit on $\tau_{d}$. In some cases, however, the count rate $c_{s}$ at time $t_{s}$ was so far below $e_{f}$ that the upper limit on $\tau_{d}$ is too large to be of much physical value. In such a case, we computed the ratio of $p_{f}-q$ to $c_{s}-q$ and then derived the natural $\operatorname{logarithm} n_{l}$ of this ratio. Assuming an $e$-folding process in the decay, the number of $e$-folding times that have occurred between flare peak time $t_{f}$ and time $t_{s}$ is just equal to $n_{l}$. This allows us to get a more meaningful value of $\tau_{d}=\left(t_{s}-t_{f}\right) / n_{l}$.

The decay time values that we derived are listed in Tables 2 and 3 .

\subsection{Estimates of Emission Measure in a Flare}

If all of the photons detected by the EUVE DS instrument originated in a volume of gas where the temperature was confined to a narrow range, and if the elemental abundances in that volume were known, then it would be a relatively simple matter to derive a more or less reliable estimate of the EM of the emitting gas from the observed count rate CR. For a star at distance $d$, $\mathrm{EM}$ would be proportional to the product $d^{2} \mathrm{CR}$, i.e., $\mathrm{EM}=$ $f(T) d^{2} \mathrm{CR}$. To the extent that the emitting gas departs from isothermal conditions in any particular flare, the proportionality constant would be expected to take on somewhat different values, depending on how different temperatures are weighted in the emission measure distribution (EMD). In such a case, the proportionality constant $f(T)$ would depend on some "effective mean temperature" of the emitting plasma. The accuracy of the numerical value that can be estimated for $f(T)$ depends on at least two factors: (1) how reliably are the atomic constants known and (2) how well do we know the elemental abundances (especially iron) in the emitting plasma? As a result, we do not anticipate that we will be able to estimate EM with high precision. We do estimate the uncertainties in what follows. 
TABLE 2

F, G, K Stars: Count Rates, EM, and $e$-Folding Times

\begin{tabular}{|c|c|c|c|c|}
\hline Data Set & $\begin{array}{c}\text { Mean Quiescent Count Rate } \\
\left.\text { (photons s }{ }^{-1}\right)\end{array}$ & $\begin{array}{l}\text { Flare Peak Count Rate } \\
\quad\left(\text { photons s }^{-1}\right)\end{array}$ & $\begin{array}{c}\text { Emission Measure } \\
\left(\mathrm{cm}^{-3}\right)\end{array}$ & $\begin{array}{l}\text { Decay Times } \\
\text { (s) }\end{array}$ \\
\hline \multirow[t]{2}{*}{ HR $12095 \ldots \ldots \ldots \ldots \ldots . . .}$. & 0.1 & 0.79 & $6.15 \mathrm{E} 52$ & 2700 \\
\hline & 0.1 & 0.75 & $5.79 \mathrm{E} 52$ & 1300 \\
\hline \multirow[t]{3}{*}{ 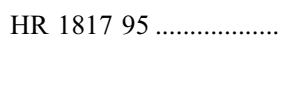 } & 0.18 & 0.25 & $1.68 \mathrm{E} 51$ & 10000 \\
\hline & $\ldots$ & 0.32 & $3.35 \mathrm{E} 51$ & 8000 \\
\hline & $\ldots$ & 0.30 & $2.87 \mathrm{E} 51$ & 17000 \\
\hline \multirow[t]{3}{*}{$\sigma^{2} \mathrm{CrB} 94 \ldots \ldots \ldots \ldots$} & 0.89 & 4.54 & $7.03 \mathrm{E} 52$ & 1100 \\
\hline & $\ldots$ & 2.61 & $3.31 \mathrm{E} 52$ & 880 \\
\hline & $\ldots$ & 2.38 & $2.89 \mathrm{E} 52$ & 420 \\
\hline 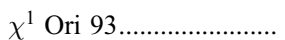 & 0.162 & 0.254 & $2.85 \mathrm{E} 50$ & 6000 \\
\hline \multirow{2}{*}{ 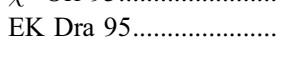 } & 0.086 & 0.295 & $9.8 \mathrm{E} 51$ & 3970 \\
\hline & & 0.174 & 4.1E51 & 3040 \\
\hline 44 Boo 94 & 0.3 & 0.75 & $2.54 \mathrm{E} 51$ & $<5500$ \\
\hline 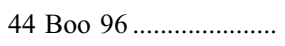 & 0.3 & 0.603 & $1.71 \mathrm{E} 51$ & $860-5300$ \\
\hline 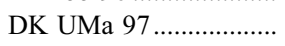 & 0.1 & 0.214 & $2.86 \mathrm{E} 51$ & $10700-14700$ \\
\hline$\kappa$ Cet $94 \ldots \ldots \ldots \ldots \ldots \ldots$ & 0.07 & 0.208 & $5.12 \mathrm{E} 50$ & $1700-5500$ \\
\hline \multirow[t]{3}{*}{$\kappa$ Cet $95 \ldots \ldots \ldots \ldots \ldots \ldots \ldots$} & 0.1 & 0.264 & $6.09 \mathrm{E} 50$ & $4100-5300$ \\
\hline & $\ldots$ & 0.174 & $2.75 \mathrm{E} 50$ & $5400-8900$ \\
\hline & $\ldots$ & 0.178 & $2.89 \mathrm{E} 50$ & $\sim 6400$ \\
\hline \multirow[t]{2}{*}{ ER Vul 95 ........................ } & 0.16 & 0.30 & $1.4 \mathrm{E} 52$ & $800-3600$ \\
\hline & $\ldots$ & 0.285 & $1.3 \mathrm{E} 52$ & $<6000$ \\
\hline 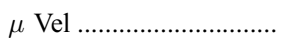 & 0.18 & 0.34 & $8.56 \mathrm{E} 51$ & 96600 \\
\hline 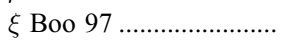 & 0.230 & 0.411 & $3.3 \mathrm{E} 50$ & 11200 \\
\hline 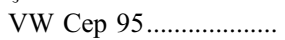 & 0.087 & 0.425 & $1.1 \mathrm{E} 52$ & $1500-3600$ \\
\hline 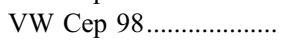 & 0.095 & 0.702 & $1.9 \mathrm{E} 52$ & 1800 \\
\hline \multirow[t]{2}{*}{ 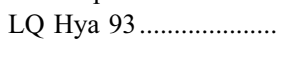 } & 0.142 & 0.368 & 3.4E51 & $\sim 5800$ \\
\hline & $\ldots$ & 0.608 & 7.69E51 & 4800 \\
\hline 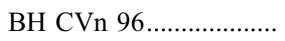 & 0.14 & 0.333 & $2.22 \mathrm{E} 52$ & $<19600$ \\
\hline AR Lac $00 \ldots \ldots \ldots \ldots \ldots$ & 0.5 & 2.5 & $1.44 \mathrm{E} 53$ & $\sim 12000$ \\
\hline \multirow[t]{3}{*}{ 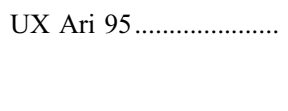 } & 0.322 & 1.465 & $1.2 \mathrm{E} 53$ & 6400 \\
\hline & 2.0 & 3.22 & $1.3 \mathrm{E} 53$ & 17400 \\
\hline & 1.5 & 2.81 & $1.4 \mathrm{E} 53$ & 33000 \\
\hline 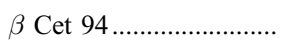 & 0.46 & 1.08 & $2.2 \mathrm{E} 52$ & 34200 \\
\hline HD $37394 \ldots \ldots \ldots \ldots \ldots \ldots \ldots$ & 0.033 & 0.060 & $1.6 \mathrm{E} 50$ & 1000 \\
\hline \multirow[t]{2}{*}{ 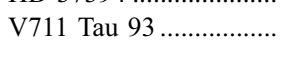 } & 1.0 & 3.06 & $7.1 \mathrm{E} 52$ & 18000 \\
\hline & $\ldots$ & 3.09 & $7.2 \mathrm{E} 52$ & 12400 \\
\hline \multirow[t]{3}{*}{ V711 Tau 94 ..................... } & 0.65 & 1.371 & $2.5 \mathrm{E} 52$ & 22200 \\
\hline & 0.7 & 1.04 & $1.2 \mathrm{E} 52$ & 11000 \\
\hline & 0.8 & 1.96 & $4.0 \mathrm{E} 52$ & 21800 \\
\hline 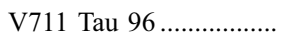 & 0.7 & 11.231 & $1.8 \mathrm{E} 52$ & 12000 \\
\hline 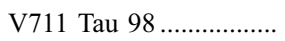 & 0.65 & 1.927 & $4.4 \mathrm{E} 52$ & 5800 \\
\hline 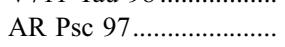 & 0.16 & 0.236 & $8.99 \mathrm{E} 50$ & $\sim 15400$ \\
\hline$\sigma$ Gem $98 \ldots \ldots \ldots \ldots \ldots$ & 0.72 & 5.90 & $3.0 \mathrm{E} 53$ & 79200 \\
\hline$\sigma$ Gem $99 \ldots \ldots \ldots \ldots \ldots$ & 0.693 & 0.953 & $1.5 \mathrm{E} 52$ & 23400 \\
\hline AB Dor $93 \ldots \ldots \ldots$ & 0.294 & 0.696 & $3.7 \mathrm{E} 51$ & 4600 \\
\hline \multirow[t]{4}{*}{ AB Dor 94} & 0.35 & 1.311 & $8.85 \mathrm{E} 51$ & $<6100$ \\
\hline & $\ldots$ & 0.741 & $3.6 \mathrm{E} 51$ & $<3200$ \\
\hline & $\ldots$ & 0.762 & $3.79 \mathrm{E} 51$ & $1250-5250$ \\
\hline & $\ldots$ & 0.912 & $5.17 \mathrm{E} 51$ & $1020-5100$ \\
\hline \multirow[t]{3}{*}{ 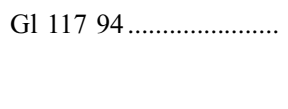 } & 0.092 & 0.143 & $1.44 \mathrm{E} 50$ & 270 \\
\hline & $\ldots$ & 0.196 & 2.94E50 & 800 \\
\hline & $\ldots$ & 0.167 & 2.12E50 & $<4500$ \\
\hline 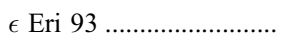 & 0.203 & 0.333 & $5.5 \mathrm{E} 49$ & 5200 \\
\hline \multirow[t]{2}{*}{$\epsilon$ Eri 95} & 0.318 & 0.486 & $7.1 \mathrm{E} 49$ & 5400 \\
\hline & $\ldots$ & 0.571 & $1.1 \mathrm{E} 50$ & 5400 \\
\hline PW And 95 & 0.095 & 0.290 & $3.8 \mathrm{E} 51$ & 5000 \\
\hline & $\ldots$ & 0.278 & $3.6 \mathrm{E} 51$ & 2900 \\
\hline & $\cdots$ & 0.261 & 3.3. 51 & 600 \\
\hline & $\ldots$ & 0.358 & $5.2 \mathrm{E} 51$ & 2600 \\
\hline & $\ldots$ & 0.289 & $3.8 \mathrm{E} 51$ & $<3900$ \\
\hline II Peg 95 & 0.4 & 1.34 & $6.9 \mathrm{E} 52$ & $17800-21000$ \\
\hline II Peg 98 & 0.3 & 0.576 & $9.5 \mathrm{E} 51$ & $\sim 10500$ \\
\hline & $\ldots$ & 0.762 & $1.59 \mathrm{E} 52$ & $<7000$ \\
\hline & & 0.779 & $1.65 \mathrm{E} 52$ & $<6400$ \\
\hline 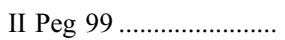 & 0.38 & 1.302 & $3.17 \mathrm{E} 52$ & $\sim 49500$ \\
\hline 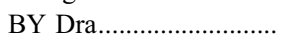 & 0.18 & 0.488 & $3.4 \mathrm{E} 51$ & $<4500$ \\
\hline & $\ldots$ & 0.411 & $2.5 \mathrm{E} 51$ & $<4800$ \\
\hline
\end{tabular}


TABLE 3

M Dwarfs: Count Rates, EM, $e$-Folding Times

\begin{tabular}{|c|c|c|c|c|}
\hline Data Set & $\begin{array}{l}\text { Mean Quiescent Count Rate } \\
\left(\text { photons s }{ }^{-1}\right)\end{array}$ & $\begin{array}{l}\text { Flare Peak Count Rate } \\
\quad\left(\text { photons s }^{-1}\right)\end{array}$ & $\begin{array}{l}\text { Emission Measure } \\
\qquad\left(\mathrm{cm}^{-3}\right)\end{array}$ & $\begin{array}{l}\text { Decay Times } \\
\text { (s) }\end{array}$ \\
\hline 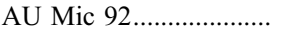 & 0.4 & 7.424 & 2.51E52 & $<5800$ \\
\hline 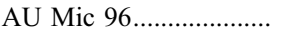 & 0.17 & 0.797 & 2.24E51 & $<5300$ \\
\hline 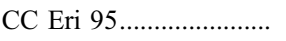 & 0.2 & 0.783 & $3.14 \mathrm{E} 51$ & $<3800$ \\
\hline \multirow[t]{3}{*}{ 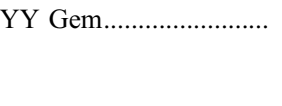 } & 0.2 & 0.59 & $4.0 \mathrm{E} 51$ & $1650-4650$ \\
\hline & $\ldots$ & 0.79 & $6.0 \mathrm{E} 51$ & $1600-4600$ \\
\hline & & 0.87 & $6.8 \mathrm{E} 51$ & $6900-9900$ \\
\hline G1 205 & 0.025 & 0.088 & 8.4E49 & $6600-9600$ \\
\hline \multirow{2}{*}{ DH Leo ................................. } & 0.176 & 0.401 & 9.4E51 & 5400 \\
\hline & $\ldots$ & 0.311 & 5.7E51 & $<4800$ \\
\hline Gl 411 & 0.024 & 0.129 & 2.79E49 & 6200 \\
\hline \multirow[t]{2}{*}{$\xi$ UMa } & 0.4 & 0.92 & $1.5 \mathrm{E} 51$ & $6000-9600$ \\
\hline & & 0.80 & $1.5 \mathrm{E} 51$ & 17000 \\
\hline Gl 644 & 0.08 & 0.84 & $1.0 \mathrm{E} 51$ & $<4000$ \\
\hline \multirow[t]{2}{*}{ FK Aqr 94....................... } & 0.25 & 0.71 & 1.4E51 & 3720 \\
\hline & 0.4 & 0.71 & $9.47 \mathrm{E} 50$ & $1800-4800$ \\
\hline \multirow[t]{6}{*}{ FK Aqr 97a ....................... } & 0.5 & 1.52 & $3.1 \mathrm{E} 51$ & $<10400$ \\
\hline & 0.4 & 1.19 & 2.4E51 & $1040-4040$ \\
\hline & 0.4 & 2.2 & $5.5 \mathrm{E} 51$ & 5720 \\
\hline & 0.4 & 2.05 & 5.04E51 & $5400-9000$ \\
\hline & 0.35 & 1.00 & $5.01 \mathrm{E} 51$ & $6000-9000$ \\
\hline & 0.4 & 0.94 & $1.65 \mathrm{E} 51$ & $1500-5100$ \\
\hline EUVE $0613-23.9 \mathrm{~B} \ldots \ldots$ & 0.01 & 3.24 & $3.0 \mathrm{E} 52$ & $<4600$ \\
\hline 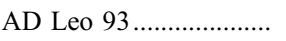 & 0.224 & 1.034 & $7.32 \mathrm{E} 50$ & 7000 \\
\hline 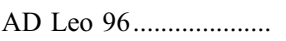 & 0.300 .770 & $4.25 \mathrm{E} 50$ & 5600 & $\ldots$ \\
\hline \multirow[t]{5}{*}{ 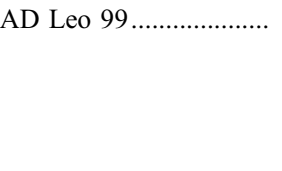 } & 0.284 & 3.088 & $2.53 \mathrm{E} 51$ & $7200-10800$ \\
\hline & 0.284 & 0.743 & $4.1 \mathrm{E} 50$ & $1000-5000$ \\
\hline & 0.283 & 1.277 & $9.0 \mathrm{E} 50$ & 4800 \\
\hline & 0.283 & 3.406 & $2.8 \mathrm{E} 51$ & $1600-5200$ \\
\hline & 0.257 & 2.269 & $1.82 \mathrm{E} 51$ & 5600 \\
\hline 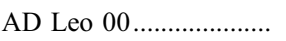 & 0.238 & 0.561 & 2.92E50 & 1000 \\
\hline \multirow[t]{2}{*}{ 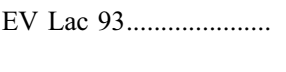 } & 0.169 & 2.239 & 2.1E51 & 1200 \\
\hline & & 0.560 & $4.0 \mathrm{E} 50$ & $<5600$ \\
\hline \multirow[t]{2}{*}{ 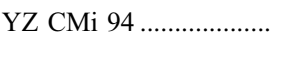 } & 0.1 & 0.500 & $6.31 \mathrm{E} 50$ & $8900-13300$ \\
\hline & & 0.487 & $6.10 \mathrm{E} 50$ & $1000-5200$ \\
\hline \multirow[t]{2}{*}{ EQ Peg 93........................ } & 0.29 & 1.18 & $1.6 \mathrm{E} 51$ & 5400 \\
\hline & & 1.65 & 2.4E51 & 6500 \\
\hline \multirow[t]{3}{*}{ 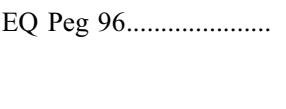 } & 0.16 & 0.512 & $6.24 \mathrm{E} 50$ & 290 \\
\hline & $\ldots$ & 0.574 & 7.34E50 & 620 \\
\hline & $\ldots$ & 0.440 & 4.97E50 & 7200 \\
\hline 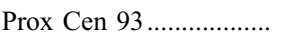 & 0.05 & 0.520 & $3.23 \mathrm{E} 49$ & $<7000$ \\
\hline \multirow[t]{5}{*}{ 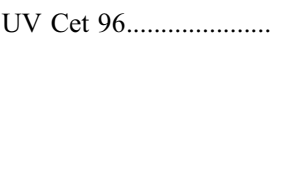 } & 0.06 & 0.550 & $1.38 \mathrm{E} 50$ & 435 \\
\hline & $\ldots$ & 0.171 & $3.13 \mathrm{E} 49$ & $<2700$ \\
\hline & $\ldots$ & 0.270 & $5.93 \mathrm{E} 49$ & $1900-6500$ \\
\hline & $\ldots$ & 0.262 & $5.70 \mathrm{E} 49$ & 120 \\
\hline & $\ldots$ & 0.206 & 4.12E49 & 610 \\
\hline \multirow{10}{*}{ 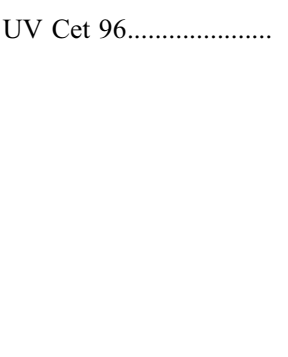 } & $\ldots$ & 0.233 & 4.88E49 & 270 \\
\hline & $\ldots$ & 0.917 & $2.42 \mathrm{E} 50$ & 125 \\
\hline & $\ldots$ & 0.234 & 4.91E49 & $1200-5200$ \\
\hline & $\ldots$ & 0.986 & 2.61E50 & 226 \\
\hline & $\ldots$ & 0.222 & 4.57E49 & $<11000$ \\
\hline & $\ldots$ & 0.548 & $1.38 \mathrm{E} 50$ & 200 \\
\hline & $\ldots$ & 0.288 & $6.44 \mathrm{E} 49$ & 165 \\
\hline & $\ldots$ & 0.267 & $5.84 \mathrm{E} 49$ & 350 \\
\hline & $\ldots$ & 0.299 & $6.75 \mathrm{E} 49$ & 270 \\
\hline & $\ldots$ & 0.355 & $8.33 \mathrm{E} 49$ & 320 \\
\hline \multirow[t]{4}{*}{ 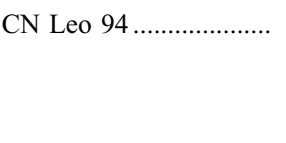 } & 0.02 & 0.252 & $5.43 \mathrm{E} 49$ & $9000-13600$ \\
\hline & $\ldots$ & 0.142 & $2.85 \mathrm{E} 49$ & $<3400$ \\
\hline & $\ldots$ & 0.111 & 2.13E49 & $<3200$ \\
\hline & $\ldots$ & 0.182 & $3.79 \mathrm{E} 49$ & $620-5120$ \\
\hline
\end{tabular}


TABLE 3-Continued

\begin{tabular}{ccccc}
\hline \hline Data Set & $\begin{array}{c}\text { Mean Quiescent Count Rate } \\
\left(\text { photons s }{ }^{-1}\right)\end{array}$ & $\begin{array}{c}\text { Flare Peak Count Rate } \\
\left(\text { photons s }^{-1}\right)\end{array}$ & $\begin{array}{c}\text { Emission Measure } \\
\left(\mathrm{cm}^{-3}\right)\end{array}$ & $\begin{array}{c}\text { Decay Times } \\
(\mathrm{s})\end{array}$ \\
\hline CN Leo 95 ………… & 0.015 & 0.057 & $9.87 \mathrm{E} 48$ & $<3200$ \\
& $\ldots$ & 0.055 & $9.38 \mathrm{E} 48$ & $3500-7500$ \\
& $\ldots$ & 0.44 & $9.94 \mathrm{E} 49$ & $114-3800$ \\
& $\ldots$ & 0.219 & $4.77 \mathrm{E} 49$ & $<1400$ \\
& $\ldots$ & 0.123 & $2.53 \mathrm{E} 49$ & $1100-5100$ \\
& $\ldots .054$ & $9.03 \mathrm{E} 48$ & $<4900$ \\
& $\ldots$ & 0.054 & $9.03 \mathrm{E} 48$ & 810 \\
& $\ldots$ & 0.109 & $2.20 \mathrm{E} 49$ & 550 \\
& $\ldots .057$ & $9.80 \mathrm{E} 48$ & $1000-5000$ \\
\hline
\end{tabular}

In order to obtain a first-order estimate of $f(T)$ for the stars that were observed to flare, we proceed as follows. The wavelength sensitivity of the DS instrument on EUVE is such that the DS response is dominated by lines of Fe XVIII-Fe XXII that are formed in the temperature range $\log T=6.8-7.1$. In view of this, it is not implausible to adopt a representative effective mean temperature for EUVE DS detection: $\log T=7.0$. With this assumption, we start by examining in detail the spectra of stars for which EUVE spectral data are available. At first, we selected four for purposes of evaluating $f(T)$ : II Peg (Griffiths \& Jordan 1998), HR 1817 (Mathioudakis \& Mullan 1999, hereafter MM99), HR 120 (Mullan \& Mathioudakis 2000), and YZ CMi (M. Mathioudakis 2005, unpublished). For each of these stars, there are enough lines with reliable identifications in the $E U V E \mathrm{SW} / \mathrm{MW} / \mathrm{LW}$ spectrometers to allow the construction of the EMD over a wide range of temperatures, from $\log T<6$ to $>7.1$. For each star, assuming solar iron abundances, we extracted from the EMD the numerical value of the EM at the effective mean temperature that is appropriate for $E U V E$ DS, i.e., $\log T=7.0$. The four stars were found to yield an average value of $4.09 \times 10^{49}$ for $f(T)$, where $d$ is in pc, CR is in photons $\mathrm{s}^{-1}$, and EM is in $\mathrm{cm}^{-3}$. Note that for the values of $d$, the distances of the stars in our sample, we use Hipparcos parallaxes where available. Others are taken from Panagi \& Mathioudakis (1993), Mitrou et al. (1997), and Maggio et al. (2000).

How large might the uncertainties in our estimate of $f(T)$ be? In a follow-up study, one of us (M. M.) has recently evaluated a total of 10 stars in the EUVE spectral database, also assuming solar iron abundances. From these stars, the values of $f(T)$ have been found to be in the range $(3-7) \times 10^{49}$, with an average of $5.4 \times 10^{49}$. Moreover, all of the EUVE DS data sets for the star $\mathrm{AD}$ Leo that we use in the present work have been analyzed independently by Sanz-Forcada \& Micela (2002). Using the quiescent EM value given by Sanz-Forcada \& Micela (2002) at $\log T=7.0$, the observed EUVE DS count rate of AD Leo in quiescence yields $f(T)=3 \times 10^{49}$.

We conclude that by adopting $f(T)=4.09 \times 10^{49}$ in our analysis, the internal uncertainties in our EM estimates are probably no more than a factor of 2 . Uncertainties in iron abundance will compound the internal uncertainties: we return to this when discussing AD Leo below. Considering the uncertainties involved with the measurement of spectral line fluxes and in the atomic data used to determine the EMD, it does not seem likely that the uncertainty in our EM estimates can be reduced below a factor of 2 or so. Fortunately, as we have already pointed out, the parameters we wish to evaluate in the present analysis are quite insensitive to errors in EM.

The stars in our sample all lie fairly close to the Sun, leading to relatively small hydrogen column densities, $N_{\mathrm{H}}$. The finite value of $N_{\mathrm{H}}$ in any particular star causes some interstellar attenuation that would affect the flux and hence the EM values. However, the effective wavelengths observed by the DS instrument $(\sim 120 \AA)$ are much shorter than the Lyman limit. As a result, attenuation effects are small. For example, for a column density typical of most of our sources, namely, $\log N_{\mathrm{H}} \approx 18.2$, the flux received at $120 \AA$ would only be reduced by $\sim 8 \%$. If the column density were to be increased to $\log N_{\mathrm{H}} \approx 18.4$, the reduction would be $\sim 13 \%$. Further details can be found in Mitrou et al. (1997). Since this is a relatively small effect, and since our results are insensitive to changes in EM, interstellar attenuation was not included as part of the EM scaling method.

The EM values we have derived for the peak of each flare (after subtracting the quiescent count rate) are listed in Tables 2 and 3 .

\section{DERIVATION OF FLARE PARAMETERS}

Applying equations (1)-(4) to the data in Tables 2 and 3, we derive four properties for flaring loops: $L, T, N_{e}$, and $B$. Results for $\mathrm{F}, \mathrm{G}$, and $\mathrm{K}$ stars are given in Table 4 . In this table, the stars span a range of luminosity classes, from V to IV to III. In Table 5, where we list the properties of loops in M stars, the stars in the sample belong to luminosity class V only. In Tables 4 and 5, we list loop lengths not only in absolute terms but also relative to the stellar radius.

\section{COMMENTS ON INDIVIDUAL STARS}

Here we examine the extent to which the results we have derived in Tables 4 and 5 are consistent with the properties that have been derived by other investigators, using a variety of methods to analyze flares from the same target stars. We are especially interested in results from analyses that are independent of the method we use here.

Because of the extent of work that has been done on flaring stars, this section of the paper is inevitably lengthy. In order to get an overview of the significance of the material in the present section, we provide a visual comparison between our results and the literature survey in tabular form in $\S 7$.

\subsection{Preamble: The FHC Test}

In what follows, when we discuss the temperature of a flare, we often refer to a certain widespread property of coronal X-ray emission, namely, a good fit to the spectrum of the quiescent corona of flare stars in general can often be achieved by using a two-temperature (2-T) model. Of course, there is in reality a distribution of temperatures present in the corona of any star, but given the limitations of signal-to-noise ratios of at least the earlier X-ray instruments, a 2-T fit can often be found that is a "good fit" in a statistical sense: the reduced $\chi^{2}$ is less than 1. 
TABLE 4

F, G, and K Stars: Calculated Flare Parameters

\begin{tabular}{|c|c|c|c|c|c|}
\hline Data Set & $\begin{array}{l}\text { Temperature } \\
\text { (MK) }\end{array}$ & $\begin{array}{l}\text { Electron Density } \\
\left(10^{11} \mathrm{~cm}^{-3}\right)\end{array}$ & $\begin{array}{l}\text { Loop Length } \\
\quad\left(10^{9} \mathrm{~cm}\right)\end{array}$ & $L / R($ star $)$ & $\begin{array}{c}\text { Minimum } B \text { Field } \\
(\mathrm{G})\end{array}$ \\
\hline \multirow[t]{2}{*}{ HR 12095} & 87 & 5.5 & 29 & 0.14 & 580 \\
\hline & 103 & 12.4 & 17 & 0.08 & 940 \\
\hline \multirow[t]{3}{*}{ HR 181795} & 26 & 0.8 & 32 & 0.28 & 120 \\
\hline & 32 & 1.1 & 32 & 0.28 & 160 \\
\hline & 26 & 0.5 & 54 & 0.47 & 90 \\
\hline \multirow[t]{3}{*}{$\sigma^{2} \mathrm{CrB} 94 \ldots \ldots \ldots \ldots \ldots \ldots$} & 113 & 15 & 15.5 & 0.18 & 1100 \\
\hline & 99 & 18 & 11 & 0.13 & 1110 \\
\hline & 115 & 40.4 & 6.1 & 0.07 & 1800 \\
\hline$\chi^{1}$ Ori $93 \ldots \ldots \ldots \ldots$ & 18.7 & 1.1 & 14 & 0.21 & 120 \\
\hline \multirow{2}{*}{ EK Dra $95 \ldots \ldots \ldots \ldots$} & 50 & 2.8 & 25 & 0.42 & 310 \\
\hline & 43 & 3.4 & 16 & 0.28 & 320 \\
\hline 44 Boo 94 ........................... & $>33$ & $>1.7$ & $<23$ & $<0.38$ & 190 \\
\hline 44 Boo 96 & $30-48$ & $1.6-13$ & $5.1-20$ & $0.09-0.33$ & $180-650$ \\
\hline 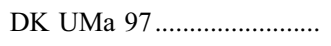 & $27-29$ & $0.6-0.8$ & $39-49$ & $0.16-0.20$ & $100-130$ \\
\hline$\kappa$ Cet 94 & $22-30$ & $1.4-5.1$ & $6.3-15$ & $0.10-0.23$ & $140-320$ \\
\hline \multirow[t]{3}{*}{$\kappa$ Cet $95 \ldots \ldots \ldots \ldots \ldots \ldots \ldots \ldots$} & $23-25$ & $1.4-1.9$ & $13-15$ & $0.20-0.23$ & $150-180$ \\
\hline & $17-19$ & $0.7-1.3$ & $13-19$ & $0.20-0.29$ & $90-130$ \\
\hline & 18 & 1.1 & 15 & 0.26 & 120 \\
\hline \multirow[t]{2}{*}{ 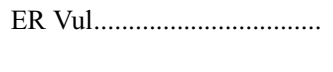 } & $56-82$ & $3.3-18$ & $8-25$ & $0.08-0.25$ & $360-1010$ \\
\hline & $>48$ & $>1.8$ & $<36$ & $<0.36$ & 250 \\
\hline 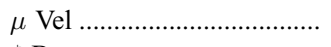 & 22 & 0.08 & 263 & 0.29 & 30 \\
\hline$\xi$ Boo..................................... & 16.6 & 0.58 & 23 & 0.38 & 80 \\
\hline VW Cep $95 \ldots \ldots \ldots$ & $53-65$ & $3.2-8.5$ & $12-23$ & $0.19-0.36$ & $340-620$ \\
\hline VW Cep 98............................ & 72 & 7.5 & 16.2 & 0.26 & 610 \\
\hline \multirow[t]{2}{*}{ LQ Hya 93} & 35 & 1.6 & 25 & 0.39 & 200 \\
\hline & 45 & 2.2 & 27 & 0.42 & 260 \\
\hline 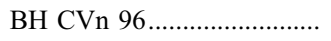 & $>41$ & $>0.5$ & $<100$ & $<0.42$ & 120 \\
\hline AR Lac 00 & 74 & 1.1 & 111 & 0.52 & 240 \\
\hline \multirow[t]{3}{*}{ 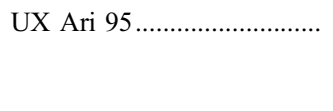 } & 83 & 2.2 & 66 & 0.17 & 360 \\
\hline & 66 & 0.74 & 143 & 0.36 & 180 \\
\hline & 57 & 0.36 & 235 & 0.58 & 120 \\
\hline$\beta$ Cet $94 \ldots \ldots \ldots$ & 36 & 0.28 & 153 & 0.15 & 80 \\
\hline HD 37394 & 25 & 8.0 & 3.2 & 0.05 & 370 \\
\hline \multirow{2}{*}{ 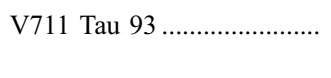 } & 56 & 0.66 & 127 & 0.47 & 160 \\
\hline & 62 & 1.0 & 96 & 0.35 & 210 \\
\hline \multirow{3}{*}{ V711 Tau 94 .............................. } & 41 & 0.46 & 114 & 0.42 & 110 \\
\hline & 41 & 0.92 & 56 & 0.21 & 160 \\
\hline & 47 & 0.49 & 127 & 0.47 & 130 \\
\hline V711 Tau 96 & 44 & 0.88 & 67 & 0.25 & 160 \\
\hline V711 Tau 98 & 66 & 2.2 & 48 & 0.18 & 320 \\
\hline AR Psc 97......................... & 20 & 0.5 & 38 & 0.16 & 80 \\
\hline$\sigma$ Gem 98 & 56 & 0.15 & 551 & 0.85 & 80 \\
\hline$\sigma$ Gem $99 \ldots \ldots \ldots$ & 36 & 0.4 & 105 & 0.16 & 100 \\
\hline AB Dor 93 & 38 & 2.1 & 22 & 0.31 & 240 \\
\hline \multirow[t]{4}{*}{ AB Dor 94} & $>44$ & $>1.7$ & $<34$ & $<0.49$ & 230 \\
\hline & $>41$ & $>3.2$ & $<17$ & $<0.24$ & 300 \\
\hline & $37-53$ & $1.8-9.2$ & $8.2-24$ & $0.12-0.34$ & $220-580$ \\
\hline & $40-60$ & $2.0-12$ & $7.7-26$ & $0.11-0.37$ & $230-710$ \\
\hline \multirow[t]{3}{*}{ Gl 11794} & 34 & 34 & 1.0 & 0.02 & 900 \\
\hline & 31 & 11 & 3.0 & 0.05 & 490 \\
\hline & $>19$ & $>1.5$ & $<10.5$ & $<0.18$ & 140 \\
\hline$\epsilon$ Eri 93 & 12.8 & 1.1 & 8.3 & 0.16 & 100 \\
\hline \multirow[t]{2}{*}{ 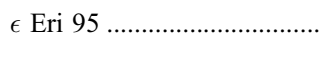 } & 13.6 & 1.1 & 9.2 & 0.18 & 100 \\
\hline & 15.0 & 1.1 & 10.1 & 0.19 & 110 \\
\hline 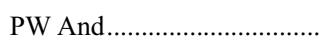 & 37 & 1.9 & 23 & 0.42 & 220 \\
\hline & 42 & 3.5 & 15 & 0.28 & 320 \\
\hline & 61 & 21 & 4.6 & 0.082 & 930 \\
\hline & 48 & 4.2 & 15 & 0.28 & 370 \\
\hline & $>40$ & $>2.6$ & $<19$ & $<0.35$ & 265 \\
\hline II Peg 95 ................................. & $54-56$ & $0.55-0.67$ & $125-141$ & $0.53-0.60$ & $140-160$ \\
\hline 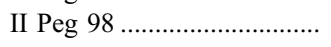 & 39 & 0.9 & 51 & 0.22 & 160 \\
\hline & $>49$ & $>1.6$ & $<43$ & $<0.18$ & 230 \\
\hline & $>51$ & $>1.8$ & $<41$ & $<0.17$ & 250 \\
\hline II Peg 99 .................................. & 36 & 0.2 & 220 & 0.93 & 70 \\
\hline BY Dra 97 & $>37$ & $>2.1$ & $<21$ & $<0.23$ & 230 \\
\hline & $>34$ & $>1.9$ & $<20$ & $<0.23$ & 210 \\
\hline
\end{tabular}


TABLE 5

M Dwarfs: Calculated Flare Parameters

\begin{tabular}{|c|c|c|c|c|c|}
\hline Data Set & $\begin{array}{c}\text { Temperature } \\
\text { (MK) }\end{array}$ & $\begin{array}{l}\text { Electron Density } \\
\quad\left(10^{11} \mathrm{~cm}^{-3}\right)\end{array}$ & $\begin{array}{l}\text { Loop Length } \\
\qquad\left(10^{9} \mathrm{~cm}\right)\end{array}$ & $L / R($ star $)$ & $\begin{array}{c}\text { Minimum } B \text { Field } \\
(\mathrm{G})\end{array}$ \\
\hline AU Mic $92 \ldots \ldots \ldots \ldots \ldots \ldots \ldots \ldots$ & $>58$ & $>2.1$ & $<42$ & $<1.08$ & 290 \\
\hline 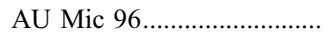 & $>32$ & $>1.7$ & $<21$ & $<0.54$ & 190 \\
\hline 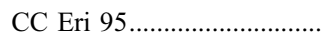 & $>38$ & $>2.6$ & $<18$ & $<0.32$ & 260 \\
\hline \multirow[t]{3}{*}{ YY Gem......................... } & $38-50$ & $2.1-6.8$ & $10-22$ & $0.24-0.52$ & $240-480$ \\
\hline & $43-56$ & $2.2-7.4$ & $11-25$ & $0.26-0.57$ & $260-530$ \\
\hline & $36-40$ & $0.96-1.4$ & $34-45$ & $0.80-1.05$ & $160-200$ \\
\hline Gl $205 \ldots \ldots \ldots \ldots \ldots \ldots \ldots \ldots \ldots$ & $12-13$ & $0.58-0.88$ & $11.1-14.7$ & $0.23-0.30$ & $70-90$ \\
\hline \multirow[t]{2}{*}{ DH Leo ............................. } & 46 & 2.0 & 31 & 0.89 & 250 \\
\hline & $>42$ & $>2.1$ & $<25$ & $<0.72$ & 250 \\
\hline 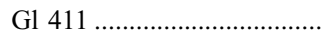 & 10.4 & 0.82 & 8.0 & 0.23 & 80 \\
\hline \multirow{2}{*}{ 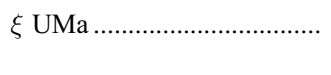 } & $25-28$ & $0.8-1.4$ & $21-30$ & $0.60-0.86$ & $120-170$ \\
\hline & 22 & 0.43 & 46 & 0.95 & 80 \\
\hline Gl $644 \ldots \ldots \ldots \ldots \ldots \ldots \ldots \ldots \ldots \ldots$ & $>29$ & $>2.1$ & $<14$ & $<0.51$ & 200 \\
\hline \multirow[t]{2}{*}{ FK Aqr $94 \ldots \ldots \ldots \ldots \ldots \ldots \ldots \ldots \ldots$} & 31 & 2.4 & 14 & 0.42 & 230 \\
\hline & $27-34$ & $1.7-5.1$ & $7.7-16.0$ & $0.22-0.46$ & $180-350$ \\
\hline \multirow[t]{6}{*}{ 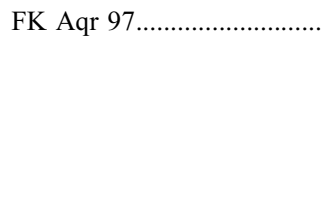 } & $>30$ & $>0.83$ & $<38$ & $<1.11$ & 130 \\
\hline & $35-49$ & $2.32-10.7$ & $6.4-17.8$ & $0.18-0.51$ & $240-610$ \\
\hline & 40 & 1.73 & 28 & 0.81 & 220 \\
\hline & $35-39$ & $1.03-1.84$ & $26.538-9$ & $0.76-1.12$ & $160-220$ \\
\hline & $35-38$ & $1.03-1.63$ & $28.7-38.9$ & $0.83-1.12$ & $160-210$ \\
\hline & $30-41$ & $1.7-6.7$ & $7.7-19.2$ & $0.22-0.55$ & $190-440$ \\
\hline EUVE $0613-23.9 B \ldots \ldots \ldots \ldots$ & $>64$ & $>2.7$ & $<37$ & $<1.51$ & 210 \\
\hline 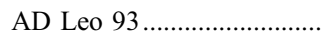 & 22.7 & 1.08 & 19.9 & 0.82 & 130 \\
\hline 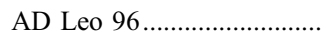 & 21.0 & 1.3 & 14.7 & 0.60 & 140 \\
\hline \multirow[t]{5}{*}{ 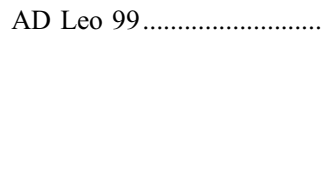 } & $28-31$ & $0.77-1.22$ & $28-34$ & $1.14-1.54$ & $120-160$ \\
\hline & $21-32$ & $1.5-9.0$ & $4.0-13$ & $0.16-0.55$ & $150-450$ \\
\hline & 26 & 1.7 & 15.8 & 0.65 & 180 \\
\hline & $34-46$ & $1.8-6.7$ & $9.2-22$ & $0.38-0.92$ & $210-460$ \\
\hline & 30 & 1,55 & 21 & 0.87 & 180 \\
\hline AD Leo $00 \ldots \ldots \ldots \ldots \ldots \ldots \ldots \ldots$ & 29 & 8.6 & 3.7 & 0.15 & 420 \\
\hline \multirow[t]{2}{*}{ EV Lac $93 \ldots \ldots \ldots \ldots \ldots \ldots \ldots \ldots \ldots$} & 46 & 8.9 & 6.9 & 0.28 & 530 \\
\hline & $>21$ & $>1.3$ & $<14.5$ & $<0.60$ & 135 \\
\hline \multirow[t]{2}{*}{ YZ CMi 94 ........................... } & $19-21$ & $0.5-0.8$ & $23-31$ & $1.1-1.5$ & $80-110$ \\
\hline & $23-35$ & $1.5-9.4$ & $4.4-15.2$ & $0.14-0.50$ & $150-480$ \\
\hline \multirow[t]{2}{*}{ 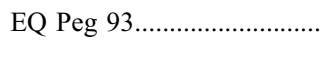 } & 29 & 1.6 & 20 & 1.5 & 180 \\
\hline & 31 & 1.4 & 25 & 1.9 & 170 \\
\hline \multirow[t]{3}{*}{ 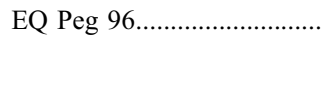 } & 48 & 38 & 1.8 & 0.14 & 1100 \\
\hline & 42 & 17 & 3.2 & 0.24 & 690 \\
\hline & 21 & 1.0 & 19 & 1.4 & 120 \\
\hline Prox Cen $93 \ldots \ldots \ldots \ldots \ldots \ldots \ldots$ & $>10$ & $>0.7$ & $<9.1$ & $<0.90$ & 70 \\
\hline \multirow[t]{5}{*}{ 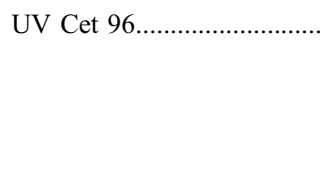 } & 30 & 20 & 1.6 & 0.15 & 640 \\
\hline & $>13$ & $>2.1$ & $<4.4$ & $<0.42$ & 140 \\
\hline & $12-17$ & $0.9-3.4$ & $4-10$ & $0.38-1.0$ & $90-200$ \\
\hline & 33 & 76 & 0.5 & 0.05 & 1300 \\
\hline & 20 & 12 & 1.6 & 0.16 & 410 \\
\hline \multirow[t]{10}{*}{ 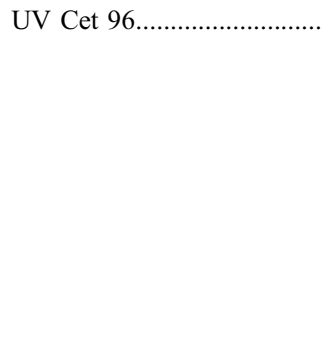 } & 26 & 30 & 0.9 & 0.09 & 740 \\
\hline & 47 & 87 & 0.7 & 0.07 & 1700 \\
\hline & $13-18$ & $1.1-5.6$ & $2.7-8.1$ & $0.26-0.78$ & $100-260$ \\
\hline & 42 & 45 & 1.2 & 0.12 & 1100 \\
\hline & $>10$ & $>0.5$ & $<14$ & $<1.3$ & 60 \\
\hline & 37 & 48 & 0.9 & 0.09 & 1100 \\
\hline & 32 & 54 & 0.7 & 0.07 & 1100 \\
\hline & 26 & 23 & 1.1 & 0.11 & 640 \\
\hline & 28 & 31 & 1.0 & 0.10 & 780 \\
\hline & 29 & 26 & 1.1 & 0.11 & 720 \\
\hline 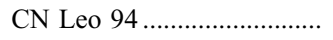 & $10.1-11.1$ & $0.4-0.6$ & $13-17$ & $1.2-1.5$ & $50-70$ \\
\hline & $>12$ & $>1.6$ & $<5.1$ & $<0.46$ & 120 \\
\hline & $>11$ & $>1.7$ & $<4.6$ & $<0.41$ & 120 \\
\hline & $12-20$ & $1.11-11$ & $1.5-7.5$ & $0.14-0.68$ & $90-400$ \\
\hline 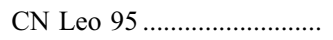 & $>9.4$ & $>1.5$ & $<3.8$ & $<0.34$ & 100 \\
\hline & $7.5-9.1$ & $0.6-1.4$ & $4.0-7.1$ & $0.36-0.64$ & $55-90$ \\
\hline & $16-39$ & $1.7-86$ & $0.6-7.6$ & $0.05-0.68$ & $140-1500$ \\
\hline & $>17$ & $>4.7$ & $<3.0$ & $<0.27$ & 240 \\
\hline & $11-16$ & $1.0-5.7$ & $2.1-6.8$ & $0.19-0.61$ & $90-250$ \\
\hline & $>8.3$ & $>0.9$ & $<5.1$ & $<0.46$ & 75 \\
\hline & 13 & 7.0 & 1.3 & 0.12 & 250 \\
\hline & 18 & 12 & 1.2 & 0.11 & 390 \\
\hline & $8.4-13$ & $0.9-5.6$ & $1.6-5.3$ & $0.14-0.48$ & $70-220$ \\
\hline
\end{tabular}


And even when multiple-temperature fits are achieved, there are often one or two peaks in the distribution that effectively play the role of one or two "components;" this is especially true of stars where the activity level is high (Guedel et al. 1997). The usefulness of 2-T fits has been demonstrated with data sets from many different spacecraft (e.g., Agrawal et al. 1981; Pasquini et al. 1989; Schmitt et al. 1990; Stern et al. 1992; Giampapa et al. 1996). Because of differences in spectral response, the various instruments do not necessarily give coincident solutions for the 2-T fits (for an example of a detailed comparison between Einstein IPC and EXOSAT see Schmitt et al. 1987b). In what follows, we use the symbol $T_{H}$ to indicate the temperature of the hotter component in a 2-T fit.

The hot component is important for the discussions in the present paper. It seems that $T_{H}$ is related to flaring processes: the more active the star (as measured by $L_{\mathrm{X}} / L_{\mathrm{bol}}$ ), the larger $T_{H}$ tends to be (e.g., Singh et al. 1999). Microwave evidence in support of a relationship between $T_{H}$ and flaring activity in dMe stars was provided by White et al. (1989): they showed that if the hot component were to occupy the low corona of a dMe star (which is "saturated" with magnetic fields that are stronger than $1 \mathrm{kG}$ ), then the corona would be optically thick at $15 \mathrm{GHz}$. When the observations did not confirm this prediction, White et al. (1989) concluded that the hot component must not lie in the low corona, but is "likely to be restricted to heights of the order of a stellar radius above the photosphere. . the hot component may be cooling flare plasma." X-ray data support the connection between $T_{H}$ and flares: when a flare occurs, a 2-T fit to X-rays indicates that the cool component appears to remain stable, while the energy release tends to occur in the hotter component (Gotthelf et al. 1994; Giampapa et al. 1996; Robrade \& Schmitt 2005). This leads us to expect that if our interpretation offlare data is to be consistent with the physics offlares, then our HSA-derived estimates offlare temperatures should exceed $T_{H}$. For brevity, we refer to this in what follows as the "flare hot component" (FHC) test.

The value of the FHC test is clear: estimates of $T_{H}$ in the literature are obtained by a completely independent approach to $\mathrm{X}$-ray data from the HSA we use here for flares. There is no a priori reason why the numerical values of $T$ derived from HSA analysis of a flare light curve should have any relationship whatsoever to the values of $T_{H}$ that emerge from spectral fits to the quiescent X-ray emission. We find, however, that in all cases where the FHC test can be applied, it is significant that not a single one of our HSA-derived $T$-values lies below $T_{H}$.

A further consistency test concerns the density. A stellar flare almost certainly involves compression of coronal material, or evaporation of denser material into the corona. Therefore, we expect that flare densities should exceed those of the quiescent corona. We apply this as a consistency test.

\subsubsection{HR $120(=H D 2726)$}

The spectral type of this star (F2) is (as far as we know) the earliest known type on which a detailed light curve of an EUV flare has been obtained (Mullan \& Mathioudakis 2000). The fact that the flaring loops appear to be quite short compared to the stellar radius $(0.08-0.14$; see Table 4$)$ supports the hypothesis of Giampapa \& Rosner (1984) that stars with shallow convection zones are expected to have short loops.

Also the fact that we see fields that are stronger (by possibly as much as an order of magnitude) in an early F star (HR 120) than in a late F star (HR 1817) supports the proposal by Giampapa \& Rosner (1984) that dynamos in early F stars would have greater amplification of field strength than in late F stars.
We have found no works in the literature that either support or refute our estimates in Table 4.

\subsubsection{HR 1817 (=HD 35850)}

Using ratios of certain EUV line intensities, MM99 obtained an upper limit to the density in the corona of this star: $N_{e} \leq$ $5 \times 10^{11} \mathrm{~cm}^{-3}$. The three flares in Table 4 have $N_{e}$ values that fall below this limit.

As regards loop lengths, assuming semicircular loops with a radius $R$, MM99 found quiescent loop lengths $(=\pi R)$ of at least $1.2 \times 10^{10} \mathrm{~cm}$. All three flaring loops listed in Table 4 have lengths that satisfy this criterion.

As regards field strengths, MM99 estimated quiescent $B$-values of $160 \mathrm{G}$ : the minimum fields in the three flares in Table 4 range from 90 to $160 \mathrm{G}$, overlapping well with the MM99 value.

In a very different sort of study, Budding et al. (2002) have obtained optical (Zeeman) and radio data for HR 1817. The Zeeman data should eventually yield magnetic field strengths on the surface, but these are not yet available. Interpreting the radio emission as gyrosynchrotron in origin, Budding et al. (2002) derived coronal magnetic field strengths of $B_{1}=62 \mathrm{G}$ and $B_{2}=107 \mathrm{G}$ for emission at two separate frequencies. These field strengths overlap well with the range of fields in the flares in Table 4. As regards loop lengths, the estimates of Budding et al. (2002) are $L_{1}=6 \times 10^{10} \mathrm{~cm}$ in the weak-field region $\left(B_{1}\right)$ and $L_{2}=3.3 \times$ $10^{10} \mathrm{~cm}$ in the strong-field region $\left(B_{2}\right)$. Interestingly, the largest flaring loop in Table 4 , with a length that is only $10 \%$ smaller than $L_{1}$, has a field limit that is only $30 \%$ larger than $B_{1}$, consistent with a dipole geometry $\left(B \sim d^{-3}\right)$. And the smaller flaring loops in Table 4, with lengths that are in close agreement with $L_{2}$, have limiting fields (119 and $158 \mathrm{G}$ ) that are not far from $B_{2}$.

As regards electron density, we cannot make any direct comparison: the radio-emitting electrons that enter into the Budding et al. (2002) analysis refer only to a population of relativistic electrons, whereas ours refers to the thermal plasma. However, since only a small fraction of the thermal electrons are expected to be accelerated to relativistic energies, it is consistent that the radio estimates yield densities that are some 4 orders of magnitude less than ours.

$$
\text { 6.1.3. } \sigma^{2} C r B(=H D 146361)
$$

Analysis of SSS data from Einstein (Agrawal et al. 1981) yielded a 2-T fit with $T_{H}$ in excess of $35 \mathrm{MK}$, with a best estimate of $52 \mathrm{MK}$. Indications of high $T_{H}$ also emerge from EXOSAT data (Pasquini et al. 1989) and Ginga data (Stern et al. 1992), where $T_{H}$ is found to be as large as 40 and $20-30 \mathrm{MK}$, respectively. These are exceptionally high temperatures for the hot component, as befits an active RS CVn system. Despite these very high $T_{H}$ values, the HSA-derived flare temperatures that we have derived for three flares are all larger than $T_{H}$, as the FHC test requires. Stern et al. (1992) specifically comment on increases in $T_{H}$ during periods of higher activity.

An X-ray flare was observed by the Einstein IPC instrument (Agrawal et al. 1986), with a decay time of $\geq 2000 \mathrm{~s}$. This is a longer decay time than occurs in any of the three flares that we analyze here. A one-temperature (1-T) spectral fit to the flare yielded $T=25 \mathrm{MK}$, cooler than we estimate here in our three flares; however, it is not consistent with the very large value of $T_{H}$ obtained from SSS data. This suggests that a 1-T fit to IPC data for this flare may not yield reliable physical information.

In the quiescent corona, a loop model interpretation of highresolution spectral line data (Agrawal et al. 1985) yielded $L=$ $1.2 \times 10^{9} \mathrm{~cm}$ and $N_{e}=5 \times 10^{10} \mathrm{~cm}^{-3}$. Both values are smaller than the flare loop lengths and densities we obtain (see Table 4). 
Doppler imaging of the surfaces of both components indicates that cool spots exist near the polar cap (Strassmeier \& Rice 2003). The maps suggest that neighboring spots are separated by no more than $\leq 15^{\circ}-30^{\circ}$ of latitude. If loops link such spots, their lengths would satisfy $L / R \leq 0.25-0.5$. This is consistent with the $L / R$ values we estimate in Table 4 .

As regards field strength, analysis of the intensity of microwave emission suggests the presence offields of about $30 \mathrm{G}$ in a region where fast electrons are present (Kuijpers \& van der Hulst 1985). The radio-emitting region is far from the stars (several stellar radii). On the surface of the star, fields are estimated to be $3000 \mathrm{G}$; these help to create fields between the stars of order $400 \mathrm{G}$ (Kuijpers \& van der Hulst 1985). The HSA-derived fields are consistent with the range 400-3000 G.

$$
\text { 6.1.4. } \chi^{1} \text { Ori (=HD 39587) }
$$

A 2-T fit yields $T_{H}=6.4-7.5 \mathrm{MK}$ (depending on the line emissivity code; Guedel et al. 1997), although EUVE data indicate the presence of Fe XXIV, at temperatures as high as $16 \mathrm{MK}$ (Haisch et al. 1994). The HSA-derived estimate of $T$ (Table 4) exceeds both of these estimates: the star passes the FHC test. We note that the "hot component" of the 2-T fit may be the dominant component in the corona: the EUVE spectra suggest that a single population of loops with $T=3.1 \mathrm{MK}$ may suffice to fit the data (van den Oord et al. 1997).

Electron densities in the corona, at temperatures of several megakelvin, are $<3 \times 10^{10} \mathrm{~cm}^{-3}$ (Ness et al. 2004). Flare density estimates in Table 4 exceed these densities, as consistency requires.

Magnetic field strengths in the photosphere are observed to be of order $1 \mathrm{kG}$ (Shi et al. 1998), and this is consistent with some theoretical estimates (1225 G; Montesinos et al. 1987). The HSA-derived coronal field strengths (Table 4) are consistent with these photospheric field strengths. There is less agreement on the spatial extent of the fields: empirical estimates (Shi et al. 1998 ) indicate that the magnetized region has an areal coverage of $60 \%$ of the surface, whereas theoretical work (Montesinos et al. 1987 ) suggests much smaller areal coverage (3.5\%). The loop size we have derived for the flare in Table $4(L / R=0.21)$ would occupy a fractional disk area that is close to the estimate by Montesinos et al. (1987). Our estimate of loop size would be consistent with the areal coverage of Shi et al. (1998) if the flaring loop occupied only a small fraction of the spotted area.

Information about loop sizes might be available from polarized radio data, and at one point, such data seemed to be available (Linsky \& Gary 1983). However, microwave emission at $6 \mathrm{~cm}$ was not confirmed by subsequent VLA ${ }^{1}$ observations (Pallavicini et al. 1985); the earlier detections must have been associated with flaring, and the polarization (possibly due to coherent processes) cannot be used to infer properties of loops.

$$
\text { 6.1.5. EK Dra (=HD 129333) }
$$

This star has been discussed recently by Ribas et al. (2005) in the context of what the Sun may have looked like at an early age. The age of EK Dra is somewhat uncertain: Ribas et al. (2005) quote an upper limit of 50-100 Myr, but they also mention that it is a member of the Pleiades moving group. The latter has an age of 20-150 Myr.

Guedel et al. (1995) have reported on an X-ray flare on EK Dra that occurred during the ROSAT all-sky survey in 1990-

\footnotetext{
1 The National Radio Astronomy Observatory is a facility of the National Science Foundation operated under cooperative agreement by Associated Universities, Inc.
}

1991. Applying radiative cooling to the light curve, the flare density was found to be about $2 \times 10^{11} \mathrm{~cm}^{-3}$, fairly consistent with the two 1995 flares in Table 4. Moreover, the source height was estimated to be $(10-20) \times 10^{9} \mathrm{~cm}$, consistent with $L$-values in Table 4 . Estimating the field strength in the cooler corona (with temperatures of a few megakelvin), Guedel et al. (1995) concluded that the field strength is at least $240 \mathrm{G}$; however, this was calculated by assuming that the field pressure exceeds gas pressure by a factor of 100 . To be consistent with the approach in the present paper, the factor of 100 must be removed: this leads to a lower limit on $B$ of $24 \mathrm{G}$ in the cool corona. This limit is consistent with the lower limits for both flares in Table 4 .

As regards coronal temperature, the quiescent corona can be fitted with a 2-T model with a hot component having $T_{H}=20-$ 21 MK (Skinner \& Walter 1998). This exceptionally high value of $T_{H}$ (associated with high levels of activity) poses a severe FHC test for EK Dra, but the HSA-estimated values of $T$ in the two flares in Table 4 exceed $T_{H}$, and so the star passes the FHC test.

A flare on EK Dra observed by $X M M$ has a flare temperature of $42 \mathrm{MK}$ (Scelsi et al. 2005). This temperature is very close to the $T$ in one of the flares in Table 4.

$$
\text { 6.1.6. } 44 \text { Boo (=HD 133640) }
$$

This is a W UMa contact binary, the brightest in the W-type subclass. The components have spectral types G2 V+G (Gondoin 2004b).

In a study of $E U V E$ spectra, Brickhouse \& Dupree (1998) used line ratios of various iron ions (Fe XIX-XXII) to estimate electron densities $N_{e}$ in the quiet corona. Values of $N_{e}$ derived from different pairs of lines turned out to be quite different, extending over the range $10^{12}-10^{14} \mathrm{~cm}^{-3}$. If the densities are indeed as high as $2 \times 10^{13} \mathrm{~cm}^{-3}$, Brickhouse \& Dupree (1998) pointed out that, given the observed EMs $\left(\mathrm{EM}=N_{e}^{2} L^{3}\right)$, the linear dimensions of the emitting structure must be very compact, $L \approx 0.004 R_{*}$ : such scales are seriously discrepant (by up to 2 orders of magnitude) with the HSA lengths in Table 4.

However, line blends are likely present in the EUVE data, and these could corrupt the above density estimates. Higher resolution spectra (obtained with XMM-Newton) allow one to identify densitysensitive line ratios where blends can be confidently ruled out (Gondoin 2004b): in the case of the helium-like O vII triplet, which is "clean and resolved" and formed at temperatures of $0.6-$ 4.6 MK, the line ratios indicate an upper limit on the density of $N_{e} \leq 8.6 \times 10^{10} \mathrm{~cm}^{-3}$. A value of $N_{e}=4 \times 10^{10} \mathrm{~cm}^{-3}$ is listed by Ness et al. (2004). (Flare density estimates in Table 4 exceed these values.) Repeating the argument of Brickhouse \& Dupree (1998) using Gondoin's upper limit on $N_{e}$, the linear size of the emitting region increases to $L \geq 0.15 R_{*}$. This is consistent with the loop sizes in Table 4.

Moreover, Gondoin (2004b) points out that quiescent loops in the 44 Boo corona may span a range of sizes. Using scaling relations for static loops, it is found that the hot loops in the quiescent corona [with $T_{H}=(7.1-8.2) \times 10^{6} \mathrm{~K}$ ] have scale sizes of $(11-23) \times 10^{9} \mathrm{~cm}$. In that case, following the discussion of Giampapa et al. (1996), flaring loops should have sizes that also lie in this range, consistent with $L$-values in Table 4 . Results for $T$ in Table 4 show that this star passes the FHC test $\left(T>T_{H}\right)$.

$$
\text { 6.1.7. DK UMa (=HD } 82210=24 U M a)
$$

This chromospherically active subgiant/giant was the subject of a magnetic field search (Marcy \& Bruning 1984). No line splitting was detected. The authors concluded that if there are fields as strong as $1 \mathrm{kG}$ on the surface, then those fields must 
occupy less than $25 \%$ of the disk. The HSA $B$-values in Table 4 , in combination with estimated $L / R_{*}$ values of 0.5 or less, are consistent with the conclusions of Marcy \& Bruning (1984).

The hot component of the corona of DK UMa (i.e., the component with $T_{H}=4-9 \mathrm{MK}$ where Ne IX is the dominant $\mathrm{Ne}$ ion) occupies $2 \%-22 \%$ of the coronal volume (Ness et al. 2004). Thus, the hot loops (where flares are expected to occur; Giampapa et al. 1996) are expected to have linear dimensions of a few tenths of $R_{*}$ and $T$ in excess of $T_{H}$. Both expectations are satisfied by the range of the results in Table 4 .

\subsection{8. $\kappa$ Cet $(=H D 20630)$}

Using the Zeeman effect in optical lines, Saar (1990) has reported a surface field of $B_{s}=1.5 \mathrm{kG}$ with a filling factor $f_{s}=0.35$, with the product $f_{s} B_{s} \approx 0.5 \mathrm{kG}$ undergoing variations of about $30 \%$ in the course of a cycle (Landi et al. 1997). Stronger fields are reported by Zhu \& Liu (1998): $B_{s}=2.6 \mathrm{kG}$ and $f_{s} B_{s}=1.8-2.1 \mathrm{kG}$.

In a separate polarimetric study, broadband polarization has been found to increase rapidly in strength toward the ultraviolet in this star, reaching almost $0.2 \%$ in the $U$ band (Huovelin et al. 1985). This type of polarization behavior may arise from Zeeman saturation in absorption lines in the spectrum: a quantitative model was reported by Mullan \& Bell (1976) for an active K6 dwarf where the observed polarization exceeds that in $\kappa$ Cet by an order of magnitude. Although no quantitative model exists for the case of a G5 dwarf (such as $\kappa$ Cet), it seems likely, based on the Mullan $\&$ Bell (1976) results, that the polarization in $\kappa$ Cet might arise from an effective photospheric field of order $0.5-1 \mathrm{kG}$.

We see that the effective surface fields derived from Zeeman and broadband polarization are consistent with the lower limits listed in Table 4.

As regards loop sizes, we note that even the loop with the largest estimated HSA length in Table $4\left(L / R_{*}=0.25\right)$ would occupy an area with a filling factor that is small compared with the surface magnetic coverage reported by Saar (1990): $f_{s}=0.35$. The latter presumably refers to an entire active region, containing many loops.

In 1986, this star was the site of a "superflare" that was revealed as a transient emission line in the D3 line of He I (Schaefer et al. 2000). The D3 line was in emission in an exposure that had a 40 minute duration but was not present in a second exposure that was started 29 minutes later. This suggests that the flare duration probably did not exceed $\sim 1 \mathrm{hr}$, unless the flare was already in progress when the first exposure started. With a 1 hour duration, the decay timescale probably did not exceed $3000 \mathrm{~s}$ : this lies in the range of decay timescales for the flares in Table 2.

In a photometric study, Messina \& Guinan (2002) have reported that this star is cyclic with a period of $5.9 \mathrm{yr}$. The amplitude of the photometric modulation is about 0.05 mag, suggesting that cool spots cover at least $5 \%$ of the surface. Such areal coverage corresponds to linear dimensions $L_{S}$ for the spotted area (active region) such that $L_{s} / R_{*}$ is at least as large as 0.2 . Individual loops that stretch from one side of the active region to the other would have loop lengths of order $0.2 R_{*}$ : this estimate agrees well with the upper limits on loop length in Table 4.

The dynamo in this star may be especially important to study: it is one of only a few stars where the surface flux of X-rays is large, but the star still shows a periodic activity cycle in the $\mathrm{Ca} \mathrm{H}$ and $\mathrm{K}$ emission lines (Hempelmann et al. 1996). Usually, such an active star would behave as an "irregular" variable as far as $\mathrm{Ca} \mathrm{H}$ and $\mathrm{K}$ is concerned, but it is in fact observed to be periodic, with a period of $5.6 \mathrm{yr}$, essentially identical to that of the photometric cycle (Messina \& Guinan 2002).
A 2-T fit to the X-ray spectrum shows $T_{H}=5.6-7.2 \mathrm{MK}$ (Guedel et al. 1997). Both flares in Table 4 have HSA-derived $T$-values in excess of this: the star passes the FHC test.

\subsubsection{ER Vul (=HD 200391)}

This system, containing two near-solar-type stars that rotate 40 times faster than the Sun, has been referred to as a "hyperactive double Sun" (Bradstreet et al. 1993). The light curve changes on very short timescales, $\sim 1$ week (Budding \& Zeilik 1987), suggesting that the starspots are relatively small. Fitting spots to the light curves indicates that the diameters of the dominant spots are $18^{\circ}$ and $22^{\circ}$ (Budding \& Zeilik 1987), i.e., $0.3 R_{*}-$ $0.4 R_{*}$. If the associated active regions are comparable in size, then loops extending from one end of the active region to the other would have linear dimensions comparable to spot diameters. These are consistent with $L / R$ values in Table 4 .

Einstein data, when fitted with 1-T models, indicate that the X-ray-emitting material has $T=12 \mathrm{MK}$ (Schrijver et al. 1984). When the data are fitted with a loop model, ER Vul (which is classified as a member of the class of "moderately active dwarfs") is found to have loops with $L \ll R_{*}$ (Schrijver et al. 1984). This is consistent with the HSA-derived loop sizes in Table 4.

Radio data indicate that there is no significant circular polarization at 4.9 or $8.5 \mathrm{GHz}$ (Rucinski 1992; Drake et al. 1992). Garcia-Sanchez et al. (2003), in a study of eight RS CVn systems at four frequencies $(1.4,4.9,8.5$, and $14.9 \mathrm{GHz})$, comment that ER Vul is the only star in their sample in which polarization could not be detected above a $3 \sigma$ level at any of their observing frequencies in any of their (four) observing sessions. This lack of polarization is unusual for RS Cvn systems (Drake et al. 1992). Although this lack may be related to orbital inclination, it is also consistent with the lack of large-scale order in the magnetic field. This could be due to the presence of only small loops in the system, each with dimensions that are $\ll R_{*}$. This is consistent with estimates of loop sizes in Table 4.

EXOSAT ME data, when fitted with 2-T models, have $T_{H}=$ $40 \mathrm{MK}$ (White et al. 1987). The 2-T fits to ROSAT PSPC data (Dempsey et al. 1993) and to $A S C A$ data (Osten et al. 2002) suggest cooler values for $T_{H}: 13.1$ and $20.4 \mathrm{MK}$, respectively. The HSA-derived $T$-values for two flares in Table 4 exceed all of these values; i.e., our results satisfy the FHC test.

As regards densities, the quiescent coronal plasma with $T \approx$ $10 \mathrm{MK}$ has a density of no more than $2 \times 10^{12} \mathrm{~cm}^{-3}$ (Testa et al. 2004a). The HSA-derived $N_{e}$ estimates in Table 4 do not exceed this limit.

$$
\text { 6.1.10. } \mu \mathrm{Vel} \text { (=HD 93497) }
$$

This is a G5 giant in orbit with a G2 dwarf. The EUVE flare that we analyze here probably occurred on the giant (Ayres et al. 1999). Using differential EMs, Ayres et al. (1999) found that the EUVE data require a peak in the flare distribution at a temperature of $20 \mathrm{MK}$. This agrees with the HSA estimate in Table 4. Using a plasmoid model, Ayres et al. (1999) estimate a density of at most $2.3 \times 10^{9} \mathrm{~cm}^{-3}$ (using $T_{7}=2$ ). The HSA estimate of density in this flare is smaller than for any other flare in our sample, but the HSA estimate is larger (by a factor of $\sim 3$ ) than the value of $N_{e}$ estimated by Ayres et al. (1999). Moreover, Ayres et al. (1999) estimated a loop length comparable to $R_{*}$, but our estimate of $L / R_{*}$ is considerably smaller than 1 .

Our density estimates are consistent with upper limits in the corona derived from Chandra data (Testa et al. 2004a).

The quiescent corona of $\mu \mathrm{Vel}$ is fitted well with a 1-T fit (Schmitt et al. 1990): 15 MK. However, a 2-T fit can also be obtained, 
and the mean $T_{H}$ reported by Schmitt et al. (1990) is $16 \mathrm{MK}$. The HSA estimate of temperature in Table 5 exceeds this, and so the star passes the FHC test.

\subsubsection{1. \& Boo (=HD 131156)}

The hot component of the quiescent corona has $T_{H}=12.6 \mathrm{MK}$ (Majer et al. 1986) or 5.01 MK (Ventura et al. 1998). The HSAderived $T$-value in Table 5 exceeds these, so the star passes the FHC test.

Using the properties of buoyant flux tubes, Montesinos et al. (1987) have estimated the magnetic field strength inside the star: $B_{c}=2115 \mathrm{G}$, occupying an area of 0.093 times the surface. With a surface field of order $2 \mathrm{kG}$, or even as weak as $1200 \mathrm{G}$ (Basri \& Marcy 1988) or $1.6 \mathrm{kG}$ (Shi et al. 1998), the coronal field estimates in Table 4 are consistent. Moreover, a fractional area of 0.093 (almost identical to the empirical area of $10 \%$ for a "starpatch;" Toner \& Gray 1988) corresponds to linear scales of about $0.3 R_{*}$, consistent with estimates of loop lengths in Table 4. However, if the magnetic flux ropes actually occupy an area of $22 \%$ (Shi et al. 1998), or as large as $40 \%$ of the surface (Basri \& Marcy 1988; Andretta \& Giampapa 1995), corresponding to linear scales of $0.5 R_{*}-0.7 R_{*}$, these would be too large to be consistent with the $L$-values we find in Table 4 . On the other hand, loop lengths determined by a loop modeling process by Ventura et al. $(1998)\left[(0.1-0.2) \times 10^{9} \mathrm{~cm}\right]$ are in serious conflict (by factors of $100)$ with our estimate in Table 4 . However, a very different estimate of loop length emerges from an analysis by Drake et al. (2000): there, with semilengths of $5.2 \times 10^{9} \mathrm{~cm}$, we find $L$ (in our notation) of order $10^{10} \mathrm{~cm}$, within a factor of $\sim 2$ of the HSA estimate in Table 4.

Drake et al. (2000) estimate densities in the quiescent corona of order $10^{10} \mathrm{~cm}^{-3}$. The HSA estimates of $N_{e}$ in Table 4 exceed this by a factor of several, as one would expect for flaring plasma.

\subsubsection{VW Cep (=HD 197433)}

This is a triple system, with a close binary (masses 0.8 and $0.3 M_{\odot}$ ) forming a W UMa system (period $=0.27$ days) and a distant third body (mass $0.6 M_{\odot}$ in a 29 yr orbit around the W UMa system; Heintz 1993). Magnetic activity is believed to be concentrated on the primary of the close binary (Kaszas et al.1998).

The 2-T fits to quiescent Einstein data (Cruddace \& Dupree 1984; Schmitt et al. 1990) and to ASCA data (Choi \& Dotani 1998) indicate a hot component with $T_{H}=35,18$, and $\approx 20 \mathrm{MK}$, respectively. An X-ray study of quiescent emission with $X M M$ (Gondoin 2004a) indicates that a three-temperature (3-T) fit is possible, with the hottest component having a temperature $T_{H}=$ 10-20 MK. The flare temperatures we derive here (see Table 4) are well in excess of these $T_{H}$ values, so the star passes the FHC test.

But the flare temperatures we derive here are considerably higher than those in an EXOSAT flare (Vilhu et al. 1988).

However, an X-ray study with Ginga (Tsuru et al. 1992) reported hard X-rays that can be fitted either as a thermal source or as a power-law source. There was no evidence of flaring. If the thermal interpretation is accepted, then the plasma is extremely hot $(T \approx 100 \mathrm{MK})$. If this indeed represents the quiescent corona, then $T_{H}$ is much larger than any other star in our sample, and our HSA estimates of flare temperatures, although among the largest we have derived (50-70 MK), would no longer satisfy the FHC test. This would be the only failure of the FHC test among the stars of our sample.

As regards loop lengths, model fits to the Ginga data led to flare lengths of $2 R_{*}-3 R_{*}$, much larger than the values we estimate using HSA.
A flare observed by $A S C A$ (Choi \& Dotani 1998) included an eclipse that led to a model-independent estimate of the linear extent of the flaring source: $5.5 \times 10^{10} \mathrm{~cm}$. This exceeds, by factors of 2-4, the HSA estimates of loop lengths in Table 4. Choi \& Dotani (1998) estimated flare density by applying radiative cooling: the result $\left(0.5 \times 10^{11} \mathrm{~cm}^{-3}\right)$ is considerably smaller than our HSA estimates. Choi \& Dotani (1998) estimated the field strength in the flare to be $>80 \mathrm{G}$, consistent with the HSA results.

Analysis of flare density (Gondoin 2004a) leads to $N_{e}>$ $6.5 \times 10^{10} \mathrm{~cm}^{-3}$, consistent with the two flares in Table 4. Analysis of flaring loop length (Gondoin 2004a) indicates $L<8 \times$ $10^{10} \mathrm{~cm}$, consistent with the values in Table 4. Estimates of flare magnetic field strength (Gondoin 2004a) are >60 G, also consistent with the HSA estimates in Table 4. As regards flare temperature, the value estimated by Gondoin (2004a) (12 MK) is certainly lower than our HSA estimates: however, we note that Gondoin's estimate of flare temperature is so low that it may lead to a violation of the FHC test.

Overall, in the case of VW Cep, we have been less successful in obtaining agreement between HSA estimates of flare parameters and the estimates of other authors than for any other stars in our sample. It may be that the physical conditions in a contact binary are such that the assumptions of the HSA method are violated to a greater extent.

\subsubsection{LQ Hya (=HD 82558)}

This is an especially interesting star in the context of dynamo theory. Donati (1999) used Zeeman-Doppler imaging to obtain maps of the surface distribution of field strength and topology for this star. The surface fields have strengths up to about $500 \mathrm{G}$, consistent with the lower limits in Table 4, and they change in shape and strength on timescales of no more than $1 \mathrm{yr}$ (McIvor et al. 2004). Donati (1999) points out that the comparable strengths of field in azimuthal and radial fields are quite different from that in the Sun: it may be that the dynamo in LQ Hya must be distributed throughout the convection zone, rather than being confined to the interface at the base of the convection zone (Kitchatinov et al. 2000). Doppler imaging evidence for cyclic spot activity with a period of $3.7 \mathrm{yr}$ has been presented by Kovari et al. (2004): this period agrees with the period predicted by Kitchatinov et al. (2000) for a distributed dynamo.

Also from Donati's paper, we note that the radial field patches along a latitude circle have opposite signs at different longitudes, which suggests that closed field lines will tend to form loops between the patches. Inspection of Donati's Figures 8-12 indicates that the length of such loops could be as large as $0.5 R_{*}$, consistent with the loop lengths in Table 4.

It was in the context of a flare on LQ Hya that Covino et al. (2001) made detailed and specific comparison between HM of flares and a simplified approach, analogous to that which we use here. Their HM analysis led to a semilength of $1.1 R_{*}-1.9 R_{*}$ for the loop in a 1992 flare. When Covino et al. (2001) applied the simpler model to the same flare data, they also found large loop lengths $0.9 R_{*}-1.8 R_{*}$. Such loops are definitely larger than even the largest estimate $\left(0.42 R_{*}\right)$ we report for the flaring loops in two 1993 flares (see Table 4).

As regards electron densities, HM yielded values of $(0.7-2) \times$ $10^{11} \mathrm{~cm}^{-3}$ for the 1992 flare (Covino et al. 2001). This range overlaps well with the range given in Table 4 for the 1993 flares. In the quiet corona, $N_{e}$ values of $10^{9.8}$ have been reported (McIvor et al. 2004): these are an order of magnitude lower than the $N_{e}$ values estimated by HSA in flaring loops.

As regards field strengths, Covino et al. (2001) estimated from the HM that a field of $300 \mathrm{G}$ would be required to confine 
the flaring coronal plasma. This is consistent with the lower limits on field strength (140-190 G) reported in Table 4.

The principal difference between our results for the 1993 flares on LQ Hya and the 1992 flare reported by Covino et al. (2001) is in the temperature: HM indicates maximum temperature of about $121 \mathrm{MK}$. Such high temperatures are certainly inconsistent with the HSA values that are listed in Table 4. It is not obvious how this inconsistency can be explained.

\subsubsection{4. $\mathrm{BH}$ CVn $(=H R$ 5110=HD 118216)}

This is a binary that has similarities to both Algol and RS CVn (Little-Marenin et al. 1986). Velocity shifts show that the active chromosphere and transition region are located on the spotted $\mathrm{K} 0$ subgiant, and not on the F2 IV star or in an accretion disk.

VLBI observations at $8.4 \mathrm{GHz}$ indicate a core-halo structure for the system. The core is centered on the $\mathrm{K}$ star and has an FWHM corresponding to a linear size of $2.6 \times 10^{11} \mathrm{~cm}$ (Ransom et al. 2003). Presumably this core represents an active region. If this region contains only a single loop, it is $2-3$ times larger than the upper limit listed in Table 4. But if the active region actually consists of more than one loop, and only one of these participated in a particular flare, then the length of any individual loop could be consistent with the HSA lengths in Table 4.

Sanz-Forcada et al. (2003), in an analysis of EMDs, conclude that structures with various temperatures exist in the corona. In the structures with $T \approx 10^{7} \mathrm{~K}$, electron densities are estimated to be $10^{12} \mathrm{~cm}^{-3}$ or more, consistent with the lower limit given in Table 4.

An optical search for surface magnetic fields resulted in an effective field of $39 \pm 18 \mathrm{G}$ (Shorlin et al. 2002). This is considerably weaker than the minimum $B$ field listed in Table 4: in order to be consistent with HSA, the magnetic region in which the flare occurred must occupy no more than about $\frac{1}{3}$ of the area of the visible disk. This is consistent with a loop length of less than $0.5 R_{*}$, as estimated by HSA.

\subsubsection{AR Lac (=HD 210344)}

The 2-T fits to ROSAT data (Dempsey et al. 1993), to $A S C A$ data (Singh et al. 1996), and to BeppoSAX data (Rodono et al. 1999) indicate that the hot component has $T_{H}=15.1,23.7$, and 25.6 MK, respectively. The flare in Table 4 has a flare $T$ in excess of all these values of $T_{H}$, as the FHC test requires.

Electron densities in the quiescent corona are estimated to be $2 \times 10^{10} \mathrm{~cm}^{-3}$ in $\mathrm{O}$ VII material (at temperatures of several megakelvin) and $8 \times 10^{11} \mathrm{~cm}^{-3}$ in the hotter Ne Ix material (Ness et al. 2004). Due to the presence of blends, Huenemoerder et al. (2003) suggest that densities cannot be estimated to better than of order $10^{11} \mathrm{~cm}^{-3}$. Densities we derive from HSA analysis (Table 4) are denser than the O VII material, and denser than those estimated by Huenemoerder et al. (2003), but not denser than the Ne IX material.

Evidence for two populations of structures in the X-ray corona was presented by White et al. (1990): a compact population had length scales that are much smaller than $R_{*}$, while the second population had length scales on the order of the binary separation. Analysis of a ROSAT flare on AR Lac led Ottmann (1993) to conclude that the flaring loop length was $3 \times 10^{11} \mathrm{~cm}$, i.e., comparable to $R_{*}$ for the active component. Further evidence to support the possibility that AR Lac contains loops extending to altitudes of about $0.5 R_{*}$ above the surface has been summarized by Siarkowski et al. (1996), although compact loops may also be present (Griffiths 1999). The linear extent of active regions on AR Lac has been determined from IUE data (Pagano et al. 2001): the angular diameter of the five distinct active regions ranges from $18^{\circ}$ to $54^{\circ}$ of longitude. These correspond to linear extents of about $0.3 R_{*}-1 R_{*}$, overlapping with the estimate of flaring loop length in Table 4. The HSA estimate of flaring loop length in Table 4 is intermediate between the lengths of these two populations.

\subsubsection{UX Ari (=HD 21242)}

Loop sizes and field strengths have been derived by Franciosini et al. (2001) for a BeppoSAX flare on UX Ari, using the two-ribbon flare model of Poletto et al. (1988). The height $H$ to which the last closed loop extends above the photosphere cannot exceed the height of the neutral point $H_{m}$ : the latter was found to be in the range $0.18 R_{*}-0.93 R_{*}$, depending on the degree of the polynomial that is used to describe the surface field. Loop lengths are larger than $H$ by a factor of $\pi$. The range of loop lengths estimated with this model overlaps with the $L / R$ values in Table 4.

Independent evidence for large loops in UX Ari is provided by radio data (Trigilio et al. 1998): orbitally modulated data can be used to estimate the size of the radio sources, which are believed to be located on large loops. (The geometry of the loops can even be distinguished: there are meridional loops and equatorial loops corresponding to polar and equatorial spots [Franciosini et al. 1999].) The radio source regions have linear extents of $(1-4) \times 10^{11} \mathrm{~cm}=0.25 R_{*}-1 R_{*}$, and they are centered at heights of $0.2 R_{*}$ above the stellar photosphere. These length scales are consistent with the loop lengths we estimate using HSA (see Table 4).

Further independent evidence for large loop sizes also comes from analysis of $A S C A / E U V E$ data (Guedel et al. 1999): in order to explain the flare data, loop sizes must be large, comparable to a stellar radius in extent.

Magnetic field strengths estimated from the two-ribbon model depend on a factor $q$, the fraction of flare energy that is converted into X-rays. Poletto et al. (1988) suggest $q=0.1$. Using this, Franciosini et al. (2001) find that the maximum field strength on the stellar surface at the flare site is $B_{m}=240-1000 \mathrm{G}$. With smaller values of $q, B_{m}$ would be even larger. These surface fields are consistent with the $B$-values estimated using HSA in Table 4.

Also using the two-ribbon model, Franciosini et al. (2001) find that densities at flare maximum are found to be $6 \times 10^{10}$ $10^{11}$. In another flare, using $A S C A / E U V E$ data, densities at flare peak are found to be $10^{11} \mathrm{~cm}^{-3}$ (Guedel et al. 1999). These densities overlap with the HSA densities given in Table 4.

A 2-T fit to Einstein observations of the quiescent corona yields a hot component with $T_{H}=28 \mathrm{MK}$ (Schmitt et al. 1990). When a continuous EMD (instead of a 2-T fit) is fitted to $A S C A$ and $E U V E$ data, the distribution peaks around $25 \mathrm{MK}$ (Guedel et al. 1999), consistent with the Einstein $T_{H}$ value. These $T_{H}$ values are among the largest known in any star, as befits an active RS CVn system. During flares on UX Ari, there were larger increases in the relative intensity of high-ionization lines (up to Fe XXIV) than in any other RS CVn system (Sanz-Forcada et al. 2002). Despite the high temperatures in the quiescent corona, flare temperatures $(57-83 \mathrm{MK})$ that we have determined using HSA (see Table 4) are clearly in excess of $T_{H}$, as the FHC test requires. Flare temperatures reported by Guedel et al. (1999) are in the range 50-100 MK, overlapping with our HSA estimates.

\subsubsection{7. $\beta$ Cet $(=H D 4128)$}

Densities in the corona are $<10^{11} \mathrm{~cm}^{-3}$ at $\mathrm{O}$ vII temperatures and $(4-5) \times 10^{11} \mathrm{~cm}^{-3}$ in the hotter Ne IX material (Ness et al. 2004). Our HSA estimates of flare density (Table 4) are only $0.4 \times 10^{11} \mathrm{~cm}^{-3}$. Our densities are not consistent with the $N_{e}$ values reported by Ness et al. (2004). However, in flares observed 
in 2000 by Ayres et al. (2001), highly ionized Fe lines were fitted best with $N_{e}=10^{10}-10^{11} \mathrm{~cm}^{-3}$, consistent with the $N_{e}$ values in Table 4.

The magnetic field strength in the photosphere is $1.68 \mathrm{kG}$ (Zhu \& Liu 1998). The HSA estimates of $B$ in the corona (Table 4) are consistent with this.

A 2-T fit to $A S C A$ data indicates a hot component with $T_{H}=$ 8.2 MK (Drake et al. 1994). Other 2-T fits, involving various detectors, have been fitted (Maggio et al. 1998) by 2-T fits with $T_{H}$ ranging from 7.1 to $19.9 \mathrm{MK}$. The HSA-derived estimate of $T$ in Table $4(T=39 \mathrm{MK})$ exceeds all of the quoted values of $T_{H}$, so the star passes the FHC test.

\subsubsection{HD 37394}

This star is a member of the Local Association, also known as the Pleiades moving group, a "reasonably coherent kinematic stream of young stars with embedded clusters and associations such as the Pleiades" (Montes et al. 2001). The stars in this group are young, with ages ranging from 20 to $150 \mathrm{Myr}$, so it is hardly surprising that HD 37394 has a rotation period (10.86 days; Gaidos et al. 2000) that is considerably shorter than the solar period. HD 37394 is among the strongest chromospheric emitters of $\mathrm{Ca}$ II emission, with an activity cycle period of $3.6 \pm 0.1 \mathrm{yr}$ (Baliunas et al. 1995). However, X-ray emission is not known to be variable (Marino et al. 2002). We could find no information in the literature that would allow us to confirm or deny the numerical values we obtain.

\subsubsection{V711 Tau (=HD 22468)}

Surface magnetic strengths up to $1.0 \mathrm{kG}$ are observed in this star (Donati 1999). The HSA-derived field strengths in the flaring corona (Table 4) are consistent with the photospheric fields. Moreover, linear scales from Doppler-Zeeman imaging (Donati 1999) indicate that the surface magnetic structures have angular extents up to a few tens of degrees in latitude or longitude, corresponding to linear scales of a few tenths of $R_{*}$. These overlap with the $L / R$ values in Table 4.

The 2-T fits to EXOSAT data (Pasquini et al. 1989) and to ROSAT data (Dempsey et al. 1993) indicate $T_{H}=25-28$ and 19.9 MK, respectively. All of the HSA-derived $T$-values in Table 4 exceed these values of $T_{H}$, so the star passes the FHC test. Fits to ASCA spectra for several flares in V711 Tau (Osten et al. 2004) indicate that the EMD extends up to temperatures of $30-50 \mathrm{MK}$ during flares. During an $X M M$ flare, the EMD was found to peak at 40-50 MK (Audard et al. 2001). These estimates of flare $T$ overlap with the HSA $T$ estimates in Table 4.

Densities in the quiescent corona are estimated from Chandra data (Ness et al. 2004) to be $10^{10} \mathrm{~cm}^{-3}$ in O VII material and $(0.8-2.5) \times 10^{11} \mathrm{~cm}^{-3}$ in Ne Ix material. The HSA-derived flare densities in Table 4 are in the range $(0.44-2.2) \times 10^{11} \mathrm{~cm}^{-3}$, overlapping with the Chandra estimates. However, the filling factor derived from Chandra data (less than $1 \%$ in Ne IX material) is much less than the linear extent of the flaring loops in Table 4.

\subsubsection{AR Psc (=HD 8357)}

This is one of the few RS CVn systems (apart from II Peg) that have been observed to flare in broadband optical data (Henry $\&$ Newsom 1996). Only the K1 subgiant primary is active: the G7 V secondary shows no evidence for a chromosphere (Fekel 1996). Two X-ray flares from this system were observed by the HEAO-1 survey satellite in 1978, on January 11 and 13 (Ambruster et al. 1984). Assuming radiative decay, Ambruster et al. (1984) derived flare densities of $2 \times 10^{11} \mathrm{~cm}^{-3}$ in both flares: these are
4 times larger than the HSA estimate in Table 4 for a flare in 1997. With these densities, the observed EMs in the two 1978 flares correspond to linear scales of at least $40 \times 10^{9}$ and $60 \times$ $10^{9} \mathrm{~cm}$ : these both exceed the HSA loop length in Table 4.

By assuming magnetic confinement, Ambruster et al. (1984) estimated $B \approx 200 \mathrm{G}$ in both 1978 flares. This estimate is consistent with the lower limit in Table 4 for the 1997 flare.

$$
\text { 6.1.21. } \sigma \text { Gem (=HD 62044) }
$$

The 2-T fits to EXOSAT data (Singh et al. 1987) and to ROSAT data (Dempsey et al. 1993) yield hot components with $T_{H}$ equal to "about 40 MK" and 16.6 MK, respectively. One of the flares in Table 4 has HSA-estimated $T$-values in excess of both of these: the second flare may also exceed both values, depending on what is meant by "about 40." Thus, the star passes the FHC test, at least for the first flare.

Densities estimated by Sanz-Forcada et al. (2002) based on EUVE line ratios are in excess of $10^{12} \mathrm{~cm}^{-3}$ : our HSA estimates of flare densities (Table 4) are certainly not consistent with these values.

The photospheric magnetic field has a strength of $1.1 \mathrm{kG}$ and a fill factor of $70 \%$ (Shi et al. 1998). The field strengths estimated by HSA in the corona (Table 4) are weak enough to be consistent with the photospheric values. Moreover, the fact that the magnetized areas of the surface occupy $70 \%$ of the area corresponds to linear scales of $0.8 R_{*}-0.9 R_{*}$, consistent with the loop length for one of the flares in Table 4.

As regards the size of magnetic structures, Doppler imaging (Kovari et al. 2001) indicates that spots on the surface of $\sigma$ Gem have diameters as large as $50^{\circ}-60^{\circ}$. This corresponds to linear scales of order $R_{*}$, consistent with $L / R$ for one of the flares in Table 4.

\subsubsection{2. $A B$ Dor (=HD 36705)}

Maggio et al. (2000) applied HM to a flare observed by BeppoSAX: they concluded that the maximum height of the flaring loop above the surface is $0.3 R_{*}$. Independently, in a Chandra study, Hussain et al. (2005) have used line shifts to demonstrate that almost $20 \%$ of the X-rays are emitted by active regions that extend no more than $0.3 R_{*}$ above the surface. Converting heights to lengths $(L \approx \pi H)$, both studies point to loops with $L / R<0.9$. This conclusion is consistent with the range of loop dimensions listed in Table 4. Also relevant to the dimension of flaring loops is the filling factors of the hot coronal component: based on Chandra line ratios, Ness et al. (2004) derive filling factors of $0.18-0.23$ for AB Dor, corresponding to linear scales of up to a few tenths of $R_{*}$. Flaring loops should have comparable dimensions, consistent with the HSA derivations in Table 4.

Maggio et al. (2000) also estimate densities of order $10^{12} \mathrm{~cm}^{-3}$ in the flaring loop, consistent with the HSA results listed in Table 4 for four EUVE flares in AB Dor.

As regards field strengths, radial fields up to $800 \mathrm{G}$ are present on the surface of AB Dor (Jardine et al. 2002): such fields will project up into the low corona with strengths that are no less than $400 \mathrm{G}$ at heights of $0.48 R_{*}$ (the longest loop length for $\mathrm{AB}$ Dor in Table 4). These estimates of coronal $B$ fields are consistent with the lower limits in Table 4.

$$
\text { 6.1.23. Gl } 117 \text { (=HD 17925) }
$$

The magnetic field strength on the surface of this star is $1.5 \mathrm{kG}$, with a filling factor of 0.35 (Saar 1990). This is consistent with the lower limits on coronal field strengths in Table 4.

Henry et al. (1995) give an extended discussion of optical variability in this star, but there is no mention of flares. Even 
though the star appears in the list of flare stars prepared by Gershberg et al. (1999), we have not been able to locate any discussion of individual flares on this star in the literature. We are therefore unable to make comparisons with the remaining HSA derivations of loop properties in Table 4.

\subsubsection{4. $\epsilon$ Eri $(=H D 22049=G l 144)$}

Giampapa et al. (1985) attempted to combine IUE and Einstein data to derive a loop model. Although the data were not simultaneous and the quality of the model fits was not high, they concluded that loops in $\epsilon$ Eri have semilengths up to $3 \times 10^{9} \mathrm{~cm}$, i.e., $L$ has values up to $6 \times 10^{9} \mathrm{~cm}$. This limit is within $30 \%$ of the HSA-derived $L$-values in Table 4.

Flaring activity occurs frequently enough in $E U V E$ data that a distribution of flare energies can be derived (Audard et al. 2000). The slope of the distribution suggests that flares dominate coronal heating. Despite the importance of flares in the corona, apart from $E U V E$, there seems to be no other literature on flares in this star. Moreover, in radio emission, the star was not detected, in either quiescence or flaring, at $3.6 \mathrm{~cm}$ (Guedel 1992).

\subsubsection{5. $P W$ And (=HD 1405)}

The radius $\left(0.8 R_{\odot}\right)$ and $B-V$ color $(1.04)$ that we have used for this active K2 dwarf star are taken from Lopez-Santiago et al. (2003). The star is a fast rotator, with $v \sin i=21.5 \mathrm{~km} \mathrm{~s}^{-1}$ and a photometric period of 1.745 days. The amplitude of photometric variations is $0.087 \pm 0.004$ (Hooten \& Hall 1990). Attributing the variations to spottedness, the spots must have a linear extent of about $0.3 R_{*}$ : this is consistent with the $L / R_{*}$ values that are listed in Table 4. No other information is available in the literature concerning field strengths, densities, or temperatures of flares.

\subsubsection{Peg (=HD 224085)}

The active component is a $\mathrm{K} 2 \mathrm{IV}$ star with radius $3.4 R_{\odot}$, while the secondary is estimated to be of spectral type M0-M3 (Berdyugina et al. 1998). The variable presence of $\mathrm{TiO}$ bands allows estimates of surface coverage by cool spots: the areal coverage may be as large as $40 \%$, although other methods (photometry, Doppler imaging) suggest smaller coverage (Solanki $\&$ Unruh 2004). Areal coverage of $40 \%$ corresponds to linear scales of $0.6 R_{*}-0.7 R_{*}$, larger than the loop sizes indicated by HSA for the 1998 flares in II Peg, but consistent with the large loop in the 1999 flare.

Tagliaferri et al. (1991) have analyzed a flare observed in 1985 by EXOSAT. The peak temperature of the flare as determined from spectral fitting was found to be 37-44 MK, overlapping with one of the HSA-derived T-values in Table 4. Applying an analysis related to HSA, Tagliferri et al. (1991) estimate a loop height of $10^{11} \mathrm{~cm}$ (assuming a single loop): this is longer than any of the loops we derive in Table 4.

Doyle et al. (1992) have analyzed X-ray data obtained with Ginga during a flare in 1989. By fitting the flare X-ray spectrum, the peak temperature for the thermal flare plasma was found to be $T=62 \mathrm{MK}$, consistent with the HSA results for flares 2 and 3 in Table 4.

Doyle et al. (1992) used two models to analyze the flare light curve: a quasi-static model and a constant velocity model (where flare decay occurs due to plasma flowing out of the loop at a constant speed). They showed that the 1989 flare on II Peg is fitted best with the constant velocity model. This yields a loop length $L$ $(=\pi H)$ of $\geq 5 \times 10^{10} \mathrm{~cm}$ : this overlaps with the HSA-derived $L$-values for the flares in Table 4 .

The density obtained by Doyle et al. (1992) for the 1989 flare was $N_{e}=6.4 \times 10^{11} \mathrm{~cm}^{-3}$, consistent with flares 2 and 3 in
Table 4. In order to confine the flare plasma, a magnetic field of at least $520 \mathrm{G}$ is required in the corona. (In the photosphere, the field strength is estimated to be $2.4 \mathrm{kG}$, based on radio emission.) This coronal field is consistent with the lower limits on $B$ listed in Table 4.

Mathioudakis et al. (2003) have analyzed the same 1989 flare that Doyle et al. (1992) discussed, but from a very different perspective: they analyzed intensity oscillations that were observed during the decay phase. Two methods of analysis were applied to the oscillations in order to estimate field strengths, both quite distinct from the method we use here. The results were 600 and $1200 \mathrm{G}$ : both of these values are consistent with the HSA lower limits on $B$ obtained from four flares in 1998 and 1999 (see Table 4).

As regards $N_{e}$, Mathioudakis et al. (2003) derived $N_{e}=$ $4 \times 10^{11} \mathrm{~cm}^{-3}$ using a loop oscillation model: this is consistent with the limits that we list for flares 2 and 3 in Table 4.

The flare $T$ derived by Mathioudakis et al. (2003), based on the loop oscillation model, is very high, some $200 \mathrm{MK}$. Although formally consistent with the limits for flares 2 and 3 in Table 4, the $T$-value is certainly higher than any value we have obtained using HSA.

Mewe et al. (1997) analyzed a 1993 flare observed with EUVE: this is especially useful in order to compare with the 1998 and 1999 EUVE flares that we analyze here. Mewe et al. (1997) apply the version of quasi-static loop decay described by van den Oord \& Mewe (1989), with an extensive discussion of the limitations of the method. As regards temperature, they find $T \geq 36 \mathrm{MK}$, consistent with all four flares in Table 4.

As regards density, Mewe et al. (1997) find $N_{e}=8 \times 10^{10} \mathrm{~cm}^{-3}$; this lies in the range of $N_{e}$ values listed in Table 4. As regards loop length $L(=\pi H)$, Mewe et al. (1997) found a large value, $L=2.5 \times 10^{11} \mathrm{~cm}$, comparable to the HSA estimate for flare 4 in Table 4.

An X-ray flare on II Peg in 1999 was observed by Chandra (Huenemoerder et al. 2001). Line ratios from He-like ions of O, $\mathrm{Ne}$, and $\mathrm{Mg}$ lead to density estimates that refer to plasmas where the respective ions are dominant. However, blends may be corrupting the $\mathrm{Ne}$ and $\mathrm{Mg}$ lines. The most unblended lines (due to $\mathrm{O}$ ) yield $N_{e}=(0.4-4) \times 10^{11} \mathrm{~cm}^{-3}$, overlapping the range of $N_{e}$ derived from HSA (Table 4). Fitting the EM observed during the flare to a single loop, the loop length $L$ is found to be $0.75 R_{*}$. This lies within the range of flaring loops derived by HSA (Table 4).

Testa et al. (2004b) report on a study of resonant scattering of X-ray lines in the quiescent corona of II Peg. They find that the photons from the $\mathrm{O}$ vIII ion, emitted by material with a density of $(0.18-1.0) \times 10^{11} \mathrm{~cm}^{-3}$, must have passed through an optical path length $l_{r}$ of at least $0.95 \times 10^{10} \mathrm{~cm}$. What does such a path length correspond to? It certainly cannot correspond to $L$, the length of a loop: a photon on its way to an observer will not pass along the entire (semicircular) path of a loop. It is more likely that $l_{r}$ corresponds to a path that passes through a part of the loop. Thus, if the loop has an aspect ratio $\alpha=d / L$ (where $d$ is the diameter at the base of the loop) of 0.1-0.3 (Doyle et al. 1992), the above path length could correspond to passage of the $\mathrm{O}$ viII photons through a loop with $L=(3-10) \times 10^{10} \mathrm{~cm}$. These values overlap well with the HSA-derived lengths of flaring loops in Table 4.

$$
\text { 6.1.27. BY Dra (=HD 234677) }
$$

The 2-T fits to EXOSAT data (Schmitt et al. 1987b), to Einstein data (Schmitt et al. 1990), and to ROSAT data (Dempsey et al. 1997) yield hot components with $T_{H}=27,16$, and $14.3 \mathrm{MK}$, respectively. The HSA-derived flare temperatures in Table 4 
are in excess of all of these estimates of $T_{H}$, as the FHC test requires.

A flare observed by EXOSAT had a density of $N_{e} \approx 2 \times$ $10^{11} \mathrm{~cm}^{-3}$ and a linear scale of order $10^{10} \mathrm{~cm}$. Both quantities agree well with the HSA estimates in Table 4.

Zeeman analysis of the magnetic field on the surface of BY Dra indicates a magnitude of $2800 \mathrm{G}$, occupying $60 \%$ of the surface (Montesinos \& Jordan 1993). The fields we derive from HSA in Table 4 are certainly less than the surface field, as consistency requires. However, the loop lengths we have derived in Table 4 seem too small to be consistent with $60 \%$ surface coverage. This problem becomes exacerbated if the true coverage of the stellar surface by cool spots is even larger than the Zeeman analysis indicates (Katsova \& Tsikoudi 1993).

\subsubsection{DH Leo (=HD 86590)}

This is a triple system, with a $\mathrm{K} 0$ primary in a 1.07 day orbit around a lower mass secondary (mass ratio $=0.68$ ). The tertiary is not tied to the binary system and has spectral type late $\mathrm{K}$ or early $\mathrm{M}$ (Barden 1984). Barden indicates that the $\mathrm{H} \alpha$ emission from $\mathrm{DH}$ Leo is found to arise primarily from the secondary component. Montes et al. (1995) claim, however, that the primary dominates, although Montes et al. (1995) do not indicate how their conclusions might be affected by $\mathrm{H} \alpha$ from the tertiary.

In order to decide between the claims of Barden (1984) and of Montes et al. (1995), we note that the secondary in the system has been found to be the site of large-scale magnetic activity. Newmark et al. (1990) in their study of the orbital modulation of $\mathrm{H} \alpha$ emission reported a feature that causes extra absorption near primary eclipse. Newmark et al. (1990) interpret this feature as a long-lived prominence "extending at least several tenths of a solar radius above the secondary." There is no indication of an analogous feature near secondary eclipse. This suggests that magnetic structures on the secondary are more prominent than those on the primary. Because of this, we assume in our analysis that the flares we observe in EUVE occur on the secondary.

Orbital solutions indicate that, depending on orbital inclination, the mass of the secondary is $0.52-0.59 M_{\odot}$ (Barden 1984) or $0.58 M_{\odot}$ (Barden 1985). Compared to the masses of the components of YY Gem (both $0.6 M_{\odot}$, with spectral type dM1), the lower mass of the secondary in DH Leo suggests a spectral type of early M. Although the spectral type of the secondary is cited as K7 by Sanz-Forcada et al. (2003), it seems more consistent, on the basis of the stellar mass, to adopt a spectral type closer to M0. We assume a spectral type of M0 in our analysis, with $B-V=$ 1.4 (the average of $B-V$ colors for two M0 stars in Table 1, AU Mic and CC Eri) and $R_{*}=0.5 R_{\odot}$.

In the context of loops extending above the secondary, if we interpret the "several tenths" of Newmark et al. (1990) as 0.3$0.5 R_{\odot}$, then the height of the prominence in terms of the stellar radius becomes $0.6 R_{*}-1 R_{*}$, consistent with the HSA-derived lengths in Table 5.

A 2-T fit to ROSAT data (Dempsey et al. 1997) indicates $T_{H}=$ 20.7 MK. Both flares in Table 4 pass the FHC test.

\subsubsection{9. $\xi U M a(=H D$ 98230)}

This quadruple system has been the subject of an extensive study by Griffin (1998). The two principal components A (spectral type $\mathrm{G} 0)$ and $\mathrm{B}(\mathrm{G} 5)$ are in a 60 yr orbit with semimajor axis $a=2$ ".536 (Mason et al. 1995): EUVE observations cannot distinguish between these components. The $60 \mathrm{yr}$ orbit indicates $M(\mathrm{~A})=1.04-1.39 M_{\odot}$ and $M(\mathrm{~B})=1.20-1.59 M_{\odot}$. Both $\mathrm{A}$ and $\mathrm{B}$ are spectroscopic binaries. Star $\xi$ UMa B consists of $\mathrm{Ba}$ (spectral type $\mathrm{G} 5$ ) and $\mathrm{Bb}$ (spectral type unknown): current photometric data are not sufficient to allow unique masses to be assigned to $\mathrm{Ba}$ and $\mathrm{Bb}$. However, by indirect reasoning, Griffin (1998) estimates that the mass of $\mathrm{Bb}$ "may well be $\geq 0.5 M_{\odot}$." The activity in the system is generally attributed to component B (Cayrel de Strobel et al. 1994). Does the activity occur on the G5 star or on Bb? Griffin (1998) favors Bb because the activity indicators are extreme for a G5 star: there are strong emission cores in $\mathrm{Ca}$ II $\mathrm{H}$ and $\mathrm{K}$, and the amount of emission (filling in) in $\mathrm{H} \alpha$, as well as the level of strong absorption in He I $\lambda 10830$, is more pronounced than in any other G5 V star (Griffin 1998). Because of this, when we interpret flares in $\xi$ UMa from $E U V E$, we assign the flares to component $\mathrm{Bb}$. By comparing with, say, YY Gem, where stars with individual masses of $0.6 M_{\odot}$ have spectral types M1, we estimate that a star with mass $0.5 M_{\odot}$ has spectral type of early M (slightly later than Griffin's claim of "late K"). We adopt M0, with $B-V=1.4$ (as for DH Leo) and radius of $0.5 R_{\odot}$.

The 2-T fits to Einstein data (Schmitt et al. 1990) and to ROSAT data (Dempsey et al. 1993) yield hot components with $T_{H}=13$ and $20.6 \mathrm{MK}$, respectively. EUVE data have been fitted by a continuous EMD by Schrijver et al. (1995): the distribution extends to temperatures of 10-20 MK. Static loop models with expanding cross section have been fitted to quiescent $E U V E$ data by van den Oord et al. (1997): two loop populations are required in order to obtain a satisfactory fit for $\xi \mathrm{UMa}$, of which the hotter have $T_{H}=7 \mathrm{MK}$. The HSA-derived temperatures for flares in Table 4 exceed $T_{H}$, as the FHC test requires.

Loop lengths in the quiescent corona of $\xi \mathrm{UMa}$ are in principle available from the results of van den Oord et al. (1997). It would have been helpful, in the present context, to have access to those lengths. However, van den Oord et al. (1997) do not cite any lengths because they say that in the presence of large loop expansion their analysis leads to loop parameters that are "rather arbitrary."

Density estimates from $E U V E$ data are $5 \times 10^{12} \mathrm{~cm}^{-3}$ (Schrijver et al. 1995). Densities close to this $\left(3 \times 10^{12} \mathrm{~cm}^{-3}\right)$ have also been derived from Chandra line ratios (Testa et al. 2004a). Our HSA-derived estimates of $N_{e}$ are certainly not consistent with such high densities.

The magnetic field strength on the surface is $2990 \mathrm{G}$, with a filling factor of 0.16 (Marcy 1984). The field estimates in Table 4 are consistent with this. From the filling factor, we estimate that the linear dimensions of active regions may be of order $0.4 R_{\odot}$. This is consistent with $L / R$ values in Table 4 .

\subsubsection{0. $A U$ Mic (=HD 197481)}

In the quiescent corona of flare stars in general, it has been found that when Einstein X-ray photons are fitted with a 2-T model, a good fit to the observed spectrum can be obtained (Schmitt et al. 1990). In the case of AU Mic, the hotter component has a temperature $T_{H}=20 \mathrm{MK}$. The FHC test requires that flare temperatures should exceed $T_{H}$. In Table 5 we see that for the two flares of AU Mic, when analyzed by HSA, the results do satisfy the FHC test.

The 1992 flare of AU Mic in our Table 5 has been the subject of independent analysis of the EUVE data by Cully et al. (1993, 1994) and Hawley et al. (1995). (The flare in our Table 5 is the larger of two flares that were analyzed by Cully et al. 1993.) In their first analysis, Cully et al. (1993) assumed a typical flare temperature $(30 \mathrm{MK})$ in order to derive various flare parameters from the observed EM: using a radiative cooling time, this led to $N_{e}=6 \times 10^{11} \mathrm{~cm}^{-3}$ (consistent with our lower limit in Table 5) and $L=5 \times 10^{10} \mathrm{~cm}$ (slightly in excess of the upper limit in Table 5). In their second analysis, Cully et al. (1994) developed a coronal mass ejection model in order to fit the long-lived 
decay of the larger flare. However, they did not attempt to fit their model to the peak of the larger flare: they fitted the light curve only for times that were later than a feature they called the "knee." As a result, the parameters derived by Cully et al. (1994) cannot be meaningfully compared with the parameters we list in Table 5: the latter refer to conditions near the peak of the flare, rather than in the long decay phase.

Hawley et al. (1995) have developed a method to analyze a flare light curve based on a flare loop energy equation that includes evaporation effects. Their analysis applies specifically to the steepest part of the light curve near flare maximum: this is the period to which our analysis also applies. Hawley et al. (1995) derive a loop length $L=2.6 \times 10^{10} \mathrm{~cm}$, consistent with the HSA estimate in Table 5. Citing Cully et al. (1993), Hawley et al. (1995) adopt a peak flare temperature of $43 \times 10^{7} \mathrm{~K}$. Using this, the maximum flare pressure derived by Hawley et al. (1995) (350 dynes $\mathrm{cm}^{-2}$ ) corresponds to a flare density of $4.2 \times 10^{10} \mathrm{~cm}^{-3}$. This is too small to be consistent with the lower limit we list in Table 5 .

In an analysis of radio and $X M M$ data of a series of flares on AU Mic, using a different line of reasoning from that we use here, Smith et al. (2005) have reported magnetic field strengths in the flare-emitting regions with upper limits of $110-150 \mathrm{G}$. These limits are formally inconsistent with the lower limits we report in Table 5, but the inconsistency is not large, only about $30 \%$.

\subsubsection{Eri (=HD 16157)}

Amado et al. (2000) have analyzed a 1989 flare on CC Eri, deriving a loop length of $0.25 R_{*}$. The latter length is consistent with the upper limit $\left(0.27 R_{*}\right)$ we derive in Table 5 for a 1995 flare. And for a flare in 1990, Pan \& Jordan (1995) report a length scale of no more than $20 \times 10^{9} \mathrm{~cm}$, consistent with the upper limit in Table 5.

Quiescent radio emission from CC Eri is polarized at the $10 \%-20 \%$ level (Osten et al. 2002), indicating large-scale ordering in the stellar magnetic field. This suggests that large magnetic loops are present in the star, with scale sizes comparable to the radius. This is consistent with the HSA estimates for the largest loops in Table 5.

Pan \& Jordan (1995) discuss their flare data in the context of an MR model: this is a very different approach from that used in our HSA. (For a comparison between a reconnection model and one particular quasi-static cooling model, see Schmitt [1994]: both models do a good job of fitting the light curve of a longlived flare.) Pan \& Jordan (1995) report a minimum magnetic field strength of $252 \mathrm{G}$, in good agreement with the minimum reported in Table 5. Moreover, if radio emission is attributed to gyrosynchrotron emission, Osten et al. (2002) report that the field strength must be at least $210 \mathrm{G}$, also consistent with the minimum in Table 5. Amado et al. (2000) estimate a surface field in the kilogauss range in the flaring region, also consistent with the limits in Table 5.

Pan \& Jordan (1995) report a maximum temperature of $32 \pm$ $4 \mathrm{MK}$, which almost overlaps with the lower limit (38 MK) reported in Table 5.

\subsubsection{YY Gem (=HD 60179)}

Radio emission has been detected from YY Gem in quiescence (Linsky \& Gary 1983). Significantly, the emission is (at least at certain epochs) circularly polarized at a comparatively high level $(\sim 10 \%)$. The presence of polarization suggests that there is largescale ordering of the fields in YY Gem, consistent with our finding (see Table 5) that loops are large on YY Gem ( $L / R$ up to 1.05).

Direct evidence for radio-emitting structures in YY Gem extending to an "altitude of probably more than a stellar radius" has been provided by VLBI observations made by Alef et al. (1997). To be sure, radio emission from fast electrons is biased toward lower densities, i.e., toward greater heights (Guedel et al. 2001). Nevertheless, the source sizes implied by the VLBI data are consistent with the HSA-derived value of $L / R$ in the third flare in Table 5.

A more direct estimate of the sizes of loops in YY Gem is provided by time-resolved orbital data from XMM (Guedel et al. 2001). The quiescent corona shows structure that is confined within 1 scale height $\Lambda$ of the photosphere: using appropriated temperatures for the quiescent corona, this corresponds to $\Lambda=$ $(1-4) \times 10^{10} \mathrm{~cm}$, i.e., $0.2 R_{*}-1 R_{*}$. Chandra data also suggest that the X-ray-emitting structures in the quiescent corona extend to heights of $\approx 10^{10} \mathrm{~cm}$, leading Ness et al. (2002) to comment, "hence much larger than typical solar coronal loops." We note that the HSA-derived loop lengths listed in Table 5 for all three flares on YY Gem overlap with the above scale sizes: it seems that the three EUVE flares might have occurred on any of the structures seen by Guedel et al. (2001) or by Ness et al. (2002) in the quiescent corona. Even more directly, Guedel et al. (2001) report that there may have been an eclipse in the course of an X-ray flare on 2000 April 24/25: from an analysis of the properties of the eclipse, Guedel et al. (2001) find that the flaring structure must have extended up to a height of $\sim 0.8 R_{*}$ above the photosphere. The longitudinal extent of the flaring loop is of the same order, i.e., $(2-3) \times 10^{10} \mathrm{~cm}$, corresponding to $0.5 R_{*}-0.75 R_{*}$. Again, this estimate of flaring loop size overlaps with the HSAderived $L / R$ values in Table 5 .

An observation of a different flare on YY Gem was subsequently made by XMM and Chandra on 2000 September 29/30 (Steltzer et al. 2002). This was a short-lived event, with a decay time of $\approx 960 \mathrm{~s}$. This is definitely shorter than any of the flares we have analyzed: e.g., in comparison to the third flare in Table 3, the decay time of the XMM/Chandra flare is an order of magnitude smaller than the upper limit on the EUVE decay time. As a result, it is not surprising that Steltzer et al. (2002) derive a loop length that is smaller (by a factor of at least 5) than any of the flares in Table 5. This indicates that in YY Gem, flares can occur in short loops as well as in long loops: we see similar behavior in UV Cet and CN Leo (see below).

Indirect, and independent, evidence for large flaring loops on YY Gem is provided by Doyle et al. (1990), in their analysis of periodic behavior in flaring. Attributing the periodicity to filament oscillations, Doyle et al. (1990) derive an estimate of the diameter $D$ of the active region in which the loop is rooted: $0.23<D / R_{*}<0.46$. The height of the loop $h$ is estimated to be in the range $0.5 D-1 D$. Converting to loop length $(L=\pi h)$, this leads to $L / R=0.36-1.45$. This overlaps with the HSA-derived $L / R$ values in Table 5 .

In an independent analysis of flare energetics in the context of a two-ribbon flare model, Doyle \& Mathioudakis (1990) concluded that the loop involved in the flare must have had a length scale such that $L / R \leq 1$, i.e., loop length is comparable to the radius.

A 2-T fit to Einstein data (Schmitt et al. 1990) indicates that in the quiescent state, the hot component has $T_{H}=13 \mathrm{MK}$ in one exposure and $T_{H}=28 \mathrm{MK}$ in a second exposure. Using ROSAT data, a 2-T fit (Dempsey et al. 1997) indicates $T_{H}=$ 15.1 MK, with a $90 \%$ range from 12.7 to $17.7 \mathrm{MK}$. Using $A S C A$ data (Gotthelf et al. 1994), variations in elemental abundances are required in order to obtain acceptable 2-T fits: when such variations are included, the quiescent corona of YY Gem is found to have $T_{H} \approx 20 \mathrm{MK}$ (using Raymond-Smith emissivities). Finally, using $X M M$ data, Guedel et al. (2001) find that the 
quiescent corona of YY Gem has a distribution of temperatures up to 15-20 MK: if we regard a 2-T fit as an approximation to this more general distribution, then $T_{H} \leq 15 \mathrm{MK}$. The HSAderived $T$-values for three flares in Table 4 all exceed these estimates of $T_{H}$, as the FHC test requires.

EXOSAT spectra of two flares in YY Gem indicated a temperature of $64_{-16}^{+44} \mathrm{MK}$ for the flaring plasma (Pallavicini et al. 1990). This range overlaps with the HSA-derived $T$ estimates for the three flares in Table 5.

$A S C A$ spectra of a flare on YY Gem indicate a 2-T fit that includes a hot component with $T=30 \mathrm{MK}$ (Singh et al. 1999): this is somewhat cooler than the $T$-values we list for flares in YY Gem.

$X M M$ data for a flare (Guedel et al. 2001) indicate a flare in which the temperature reaches around $40 \mathrm{MK}$ : this overlaps with the HSA-derived $T$-values in Table 5.

As regards field strengths, Doyle \& Mathioudakis (1990) have interpreted the energy in certain flares in YY Gem in terms of a two-ribbon model. Flare energetics are found to be consistent if the surface field strength is of order $1 \mathrm{kG}$ : this is consistent with the HSA-derived estimates of (coronal) field strengths in Table 5. The latter are expected to be smaller than the surface fields.

As regards electron densities, the quiescent spectrum of YY Gem as observed by $X M M$ indicates $N_{e}$ (quies) $=(1.6-2.9) \times$ $10^{10} \mathrm{~cm}^{-3}$ (Guedel et al. 2001), with similar densities derived from Chandra data: $2.5 \times 10^{10} \mathrm{~cm}^{-3}$ (Ness et al. 2002). The HSAderived estimates offlare density (Table 5) indicate $N_{e} \geq 10^{11} \mathrm{~cm}^{-3}$, consistent with flaring plasma being denser than the quiescent corona.

\subsubsection{Gl 205 (=HD 36395)}

Magnetic field strength on the surface is $1.5 \mathrm{kG}$, occupying 0.15 of the surface (Shi et al. 1998). The field strength is consistent with the HSA-derived estimates of $B$ in Table 5. The fractional coverage of the surface suggests loop lengths of order $0.4 R_{*}$, consistent with results in Table 5.

\subsubsection{4. $G l 411$ (=HD 95735)}

No relevant data can be found in the literature for this star.

$$
\text { 6.1.35. Gl } 644(=H D 152751=\text { V1054 Oph=Wolf 630) }
$$

Astrometric data from Hipparcos suggest that this is a close binary, with components separated by 0 ". 23 and with masses of 0.41 and $0.66 M_{\odot}$ (Soderhjelm 1999). However, the quality of the astrometric solution is poor, and Soderhjelm (1999) suggests that the "component" with mass $0.66 M_{\odot}$ is actually a spectroscopic binary. The angular resolution of EUVE DS is not sufficient to separate even the astrometric pair. The combined system has a spectral type of M3 (Reid et al. 1995). Even if we do not know the masses of the individual stars in the spectroscopic binary, we will probably not be grossly in error if we regard our analysis as being relevant to an "early M" star, with an effective $B-V$ value of about 1.5 and $V-I$ of about 2.0 .

EUVE DS flare statistics have been used by Kashyap et al. (2002) to demonstrate that the X-ray-emitting corona(s) in this star is (are) heating entirely by flaring.

This star was among the first $\mathrm{M}$ dwarfs to be detected in microwave emission (Linsky \& Gary 1983). At times, the microwave emission is observed to be polarized (Linsky \& Gary 1983; Jackson et al. 1989), suggesting that the magnetic field is organized on large scales in the corona. This is consistent with the large loop size in Table 5. The altitude to which radio loops (which emit at $8-15 \mathrm{GHz}$ ) extend has been estimated as $0.8 R_{*}$ and $\sim 1 R_{*}$ (Leto et al. 2000): these loop sizes are larger than the upper limit we have obtained in Table 5 .
Independent evidence for the presence of a large structure in the corona is indicated by the presence of a long-lasting preflare dip in the light level of the star (Ventura et al. 1995): the structure (whatever it was) was large enough to influence at least $20 \%$ of the star's surface area. Such an area requires a linear extent of $0.4 R_{*}-$ $0.5 R_{*}$, consistent with the loop size in Table 5 .

The hot component of the quiescent corona has $T_{H}>8 \mathrm{MK}$ (Swank \& Johnson 1982), $T_{H}=9.12 \mathrm{MK}$ (Giampapa et al. 1996), or $T_{H}=12.3 \mathrm{MK}$ (Shi et al. 1998). In view of these numbers, the flare in Table 5 with $T>29 \mathrm{MK}$ passes the FHC test. Flares observed with EXOSAT ME were found to have $T=24$ and 36 MK (Pallavicini et al. 1990): at least one of these is consistent with the HSA estimate in Table 5.

The surface field strength times a filling factor has the value $f B \approx 1 \mathrm{kG}$ (Shi et al. 1998). For any realistic $f$-value, the surface field strengths must be a few kilogauss. This is consistent with the HSA-derived estimate of coronal field in Table 5. Also, the coronal magnetic field in the site of radio emission is 600 and $<1000 \mathrm{G}$, depending on the frequency of observation (Leto et al. 2000). These fields are also consistent with the lower limit given in Table 5.

$$
\text { 6.1.36. FK Aqr (=HD 214479) }
$$

The 2-T fits to Einstein and ROSAT data indicate a hot component with $T_{H}=12-17$ (Agrawal 1988), 14-20 (Schmitt et al. 1990), and 14 MK (Dempsey et al. 1997). All of the flare temperatures in the flares listed in Table 5 have $T$ in excess of $T_{H}$, consistent with the FHC test.

The EUV flares that enter into our sample have also been used in a very different context by Kashyap et al. (2002) to demonstrate that the corona in FK Aqr is heated by many small flares.

$$
\text { 6.1.37. EUVE 0613-23.9B }
$$

This star was discovered serendipitously to flare in an EUVE DS image (Christian et al. 2003). No other information is available in the literature.

$$
\text { 6.1.38. AD Leo }(=\text { Gl 388) }
$$

The first flare in our sample occurred during a time interval that was the subject of detailed modeling by Hawley et al. (1995) and Cully et al. (1997). Using rise and decay timescales, Hawley et al. (1995) obtained a loop length of $380 \mathrm{Mm}$ for the larger of two flares in the data. With a more refined estimate of temperature, Cully et al. (1997) obtained lengths of 240 and $470 \mathrm{Mm}$ (assuming solar abundances). These are to be compared with our estimates of $199 \mathrm{Mm}$ in Table 5: our estimated $L$-values appear somewhat shorter than the range derived by Cully et al. (1997) and Hawley et al. (1995).

However, we note that the values of EM that enter into the analysis of Cully et al. (1997) are significantly larger (by $\sim 10$ ) than the EM value we estimate in Table 3. This discrepancy arises because of the assumed value of iron abundance in the flare plasma. We have adopted solar coronal abundances in our work. We note that when Cully et al. (1997) also adopt solar coronal abundances, their estimate of EM $\left(13 \times 10^{50}\right)$ at $\log T=$ 7.0 agrees with our estimate in Table $3\left(7.32 \times 10^{50}\right)$ within a factor of 2. (This is consistent with the discussion given above concerning the "internal" uncertainties in analyzing EUVE DS data.) However, Cully et al. (1997) also examine the possibility that the iron abundance in the flaring plasma is significantly different from solar. In particular, they examine a case where the iron abundance is 0.1 times solar: this causes their estimate of EM to increase by a factor of 10 . Cully et al. (1997) point out that the $E U V E$ data do not allow one to choose between the two 
possible iron abundances in the flaring plasma. It is known that in solar flares, elemental abundances may vary from place to place in a single flare (e.g., Feldman et al. 2003). This makes it seem unlikely that we will be able to determine reliably the true iron abundance in a stellar flare.

It must be admitted that an uncertainty by a factor of 10 in the estimated value of EM is a troubling feature of the present work. Nevertheless, even in the presence of such a large uncertainty, certain aspects of our results do not change too much. Thus, according to the scalings in equations (1)-(3), when we increase EM by a factor of 10 for the 1993 flare of AD Leo, we arrive at $L=350 \mathrm{Mm}$, entirely consistent with the loop lengths derived by Hawley et al. (1995) and Cully et al. (1997).

Cully et al. (1997) have concluded that "the dominant flaring emission originates from long loops with $L \sim R_{*}$." This is consistent with our estimate $L / R=0.82$ for the first flare in Table 5 , as well as for several other flares in Table 5. If we were to use the EM of Hawley et al. (1995), we would estimate $L / R=1.45$, comparable to another flare on AD Leo in 1999 (see Table 5). More recently, application of loop modeling to $X M M$ data suggests that flaring plasma exists on loops where the semilength is $\leq 0.3 R_{*}$ (Robrade \& Schmitt 2005). This leads to loop lengths $\bar{L} \leq 0.6 R_{*}$, overlapping with the smaller flares in Table 5 .

An independent approach to loop sizes in AD Leo is provided by Giampapa et al. (1996). They find that the cool component can be contained in many small loops close to the surface, but the hot component must occupy larger structures that fill only a small fraction of the volume. Inspection of their results for AD Leo (their Fig. $3 a$ ) indicates that a consistent solution requires that the filling factor for the hot component cannot exceed $\sim 0.1$. In that case, the hot loops are found to have lengths that are $\geq s_{p}$, where the pressure scale height $s_{p}$ (for the hot component) has the value $2.9 \times 10^{10} \mathrm{~cm}$, close to $R_{*}$. Thus, in AD Leo, hot loop lengths are comparable to $R_{*}$. Moreover, Giampapa et al. (1996) explicitly state that flares in $\mathrm{dM}$ stars are likely to occur in the hot loops. Therefore, we should be able to make a direct comparison between $L_{H}$ as estimated by Giampapa et al. (1996) and the values of $L$ provided by HSA in Table 5 . We see that indeed our estimates of $L / R_{*}$ include values that are comparable to unity.

Evidence for large-scale organization of the stellar magnetic field is indicated by circular polarization in the quiescent radio emission (Jackson et al. 1989). Magnetic loops with lengths comparable to a stellar radius provide a natural source of largescale ordering in the field.

As regards densities, the density at the base of the quiescent corona is estimated to be $2.4 \times 10^{10} \mathrm{~cm}^{-3}$ (Abada-Simon et al. 1997): we expect flare densities to exceed this. Cully et al. (1997) derived peak flare densities in the range $10^{9}-10^{11} \mathrm{~cm}^{-3}$, and recent estimates of $N_{e}$ in AD Leo using Chandra data indicate that the density changes at different times, with values that range from $10^{11}$ to $10^{12} \mathrm{~cm}^{-3}$ or more (Maggio \& Ness 2005). All of these ranges overlap with the range of $N_{e}$ values listed in Table 5 for flares on AD Leo.

Photospheric magnetic fields on AD Leo are of order $3.8 \mathrm{kG}$, filling some $73 \%$ of the surface (Shi et al. 1998). The coronal fields we estimate in Table 5 are consistent with the photospheric limit. Magnetic field strengths in flares on AD Leo have been estimated from radio data (Smith et al. 2005): field strengths of order $100 \mathrm{G}$ are obtained, almost overlapping with the limits in several flares in Table 5. The large areal coverage of magnetic regions on the surface $(73 \%)$ corresponds to linear scales of $0.8 R_{*}-$ $0.9 R_{*}$, consistent with the loop lengths we list in Table 5 .

Temperatures in flares have been derived by Cully et al. (1997): in one flare, they found $T=13 \mathrm{MK}$, while in a second flare, they found 16 (with solar abundances) or $<32$ MK (assuming 0.1 times coronal abundances). The range of $T$ we have obtained from HSA (Table 5) extends from 21 to $46 \mathrm{MK}$, overlapping with the range of Cully et al. (1997). In the quiescent corona, a 2-T fit indicates $T_{H}=8.9-10.0 \mathrm{MK}$ (Giampapa et al. 1996): the flare $T$-values in Table 5 all satisfy the FHC test.

$$
\text { 6.1.39. EV Lac }(=\text { Gl 873) }
$$

This is an active flare star with a rotational period of 4.376 days, on which spots survive for a month or two (Contadakis 1995).

In quiescence, the corona has a hotter component that is variously reported as $T_{H}=9.2$ (Osten et al. 2005; Chandra data) and 20 MK (Favata et al. 2001; ASCA data). In either case, both of our HSA estimates offlare temperature in Table 4 satisfy the FHC test.

Flare temperatures have been obtained by a variety of observations. Using BeppoSAX, Sciortino et al. (1999) found that in EV Lac, flare plasma has a temperature of about $40 \mathrm{MK}$, consistent with both HSA estimates in Table 5. In a sample of six flares, Osten et al. (2005) found that the flare temperature (obtained from spectral fits) ranged from 10 to $30 \mathrm{MK}$. One of the HSA estimates in Table 4 is consistent with this range. Data from $X M M$ have been subjected to six-temperature (6-T) fits (Robrade \& Schmitt 2005), showing a peak in EM at $T=7-8 \mathrm{MK}$, as well as a smaller peak at $T=20-30 \mathrm{MK}$. During a flare, the EM increases most significantly in the range $T=15-40 \mathrm{MK}$. This range overlaps with the HSA estimates of flare temperature in Table 4.

As regards loop lengths, the literature contains a wide range of $L / R_{*}$ values. In a flare observed by HEAO-1, Ambruster et al. (1984) use an HSA-like approach to obtain a loop length of $5 \times 10^{9} \mathrm{~cm}$, consistent with the HSA estimate for the second flare in Table 5, and close to the HSA estimate for the first flare in Table 5. In a flare observed by BeppoSAX, Sciortino et al. (1999) reported loops with lengths no longer than $0.1 R_{*}$. This is shorter than either of our HSA estimates in Table 5. A flare observed by $A S C A$, when analyzed by HM (Favata et al. 2001), is found to have a loop length of $\sim R_{*}$, which Favata et al. (2001) describe as "relatively compact." Nevertheless, this result exceeds the loop lengths we have derived by HSA for the two flares in Table 5. A very large radio flare (Osten et al. 2005) also had a length comparable to $R_{*}$ : this is larger than our HSA estimates in Table 4. Loop lengths in the flaring plasma (i.e., at the hottest temperatures) have values up to $0.3 R_{*}$ (Robrade \& Schmitt 2005), consistent with the HSA estimates of $L / R_{*}$ in Table 4.

It seems that our HSA estimates are neither large enough nor small enough to be consistent with the loop lengths that have previously been reported. This may simply reflect the presence of a wide range of loop lengths in stars such as EV Lac, which belongs to the cool subsample of stars in Figure 1 (below).

As regards densities, Ambruster et al. (1984), using an HSAlike approach, report densities in the range $>(4-10) \times 10^{11} \mathrm{~cm}^{-3}$. Favata et al. (2001), applying HM to a flare, obtain $N_{e}=(2-$ 20) $\times 10^{11} \mathrm{~cm}^{-3}$. Our HSA estimates in Table 5 for both flares are consistent with these densities.

As regards magnetic field strengths, values of at least $60-110 \mathrm{G}$ were reported in a large radio flare by Osten et al. (2005): these are consistent with HSA estimates. Also, photospheric fields of $3.8 \pm 0.5 \mathrm{kG}$ occupy about one-half of the surface of EV Lac (Johns-Krull \& Valenti 1996). Our HSA estimates offield strengths in the flaring plasma are consistent with the photospheric field strengths.

$$
\text { 6.1.40. YZ CMi (=Gl 285) }
$$

VLBI observations (Pestalozzi et al. 2000) have resolved a circularly symmetric component that indicates that the corona 
extends to heights of $0.7 R_{*} \pm 0.3 R_{*}$ above the photosphere. This suggests that loops with lengths comparable to $1 R_{*}$ are present in the corona. This is consistent with the loop length of $0.75 R_{*}-$ $1.01 R_{*}$ listed for one of the flares in Table 5 .

A 2-T model applied to Einstein data (Schmitt et al. 1990) indicates a hot component with $T_{H}=16-25 \mathrm{MK}$. The HSA-derived $T$-values in Table 5 may exceed $T_{H}$, provided that the "true" value of $T_{H}$ is closer to the lower end of the above range.

\subsubsection{EQ Peg (=Gl 896)}

Both components of this system (M3.5 Ve+M5e) undergo flares, with flares on one component sometimes appearing to trigger flares on the other (Rodono 1978). There is evidence that EQ Peg B is the more active flare star (e.g., Robrade et al. 2004). in view of this, we assume that the EUVE flares occurred on EQ Peg B.

For the two 1993 flares we analyze in this paper, we use the light curves that were published by Monsignori Fossi et al. (1995).

Topka \& Marsh (1982) interpreted microwave radiation from the quiescent system in terms of thermal gyroresonance emission. This requires a field $B \geq 300 \mathrm{G}$ that exists on length scales of order $2 R_{*}$ in the system. These fields are consistent with the limits in Table 5.

Baliunas \& Raymond (1984) applied the HSA to a 1981 flare on EQ Peg B that was observed with $I U E$ : they found $T=70 \mathrm{MK}$, $L=5 \times 10^{10} \mathrm{~cm}$, and $N_{e}=2 \times 10^{11} \mathrm{~cm}^{-3}$. Neither the $T$ nor the $L$ estimate overlaps with the values we find in Table 5 .

A 1-T fit to EXOSAT ME data obtained during a 1984 flare yielded $T=26 \mathrm{MK}$ (Haisch et al. 1987). Assuming radiative cooling only, a flare density $N_{e}=2 \times 10^{11} \mathrm{~cm}^{-3}$ was derived. Then the EM was converted to an equivalent linear scale, depending on the number of loops that are involved in the flare: $L=$ $\pi h=(20-30) \times 10^{9} \mathrm{~cm}$. These results overlap with the ranges of $T$ and $N_{e}$ values in Table 5 and almost overlap with the $L$-values.

A subsequent comprehensive survey of EXOSAT results (Pallavicini et al. 1990) indicated that EXOSAT ME spectra during a 1985 flare on EQ Peg could be fitted with a 1-T model with $T=42 \mathrm{MK}$. Both of the EXOSAT ME flare temperatures overlap with the HSA-derived T-values for the three 1996 EUVE flares in Table 5.

Using EXOSAT observations of the quiescent corona, Pallavicini et al. (1988) obtained a 2-T fit in which the hot component has $T_{H}=14 \mathrm{MK}$. When Einstein data are subjected to a 2-T fit (Schmitt et al. 1990), the result is $T_{H}=18-22 \mathrm{MK}$. We therefore expect flare temperatures to exceed 14-22 MK. At least two of the HSA estimates in Table 5 satisfy this criterion.

Inclusion of multiple-temperature components has become possible as more refined data become available: 3-T and 6-T fits have been presented very recently by Robrade \& Schmitt (2005), with $T$-values going up to several tens of megakelvin. Robrade \& Schmitt (2005) find that the hottest material occupies loops with lengths up to roughly $0.3 R_{*}$. Since it is the hottest loops that are expected to be the site of flare activity, this suggests that the lengths of flaring loops should also have values up to roughly $0.3 R_{*}$. This is consistent with the HSA derivations in Table 5.

Using an entirely different approach, Poletto et al. (1988) applied an MR model to a large 1985 flare observed by EXOSAT: the decay time was $7500 \mathrm{~s}$, comparable to the longest flare in Table 5 . They obtained $N_{e} \geq 8 \times 10^{11}$ and $B \geq 1200 \mathrm{G}$, both consistent with the results in Table 5. However, their estimate of the loop length in the flare was quite large, up to $4 \times 10^{10} \mathrm{~cm}$, exceeding $R_{*}$ : this is a factor of at least 2 larger than the largest EQ Peg loop in Table 5. Independent evidence for the presence of large loops (exceeding $R_{*}$ ) in EQ Peg has been provided by radio data (Kundu et al. 1987). As regards flare temperatures, spectral fits to the EXOSAT ME data led Poletto et al. (1988) to a peak $T=44 \mathrm{MK}$, overlapping with the range of $T$-values in Table 5.

VLBI radio data obtained during a flare indicate that the flare is no larger than $49 \times 10^{9} \mathrm{~cm}$ in extent (Benz et al. 1995). This is consistent with the lengths of all three $L$-values in Table 5.

\subsubsection{Prox Cen (=Gl 551)}

Reale et al. (1988) subjected an X-ray flare on Prox Cen in 1980 to HM analysis. They obtained peak temperatures of 35$42 \mathrm{MK}$, consistent with the lower limit in the 1993 flare in Table 5. As regards loop length, Reale et al. (1988) found $L=1.4 \times$ $10^{10} \mathrm{~cm}$, a few times larger than Haisch (1983) derived in his original work. The HM value of $L$ in the 1980 flare is within $10 \%-20 \%$ of the HSA value of $L$ in the 1993 flare in Table 5. The peak plasma densities in various models that Reale et al. (1988) tried were in the range $(5-13) \times 10^{10} \mathrm{~cm}^{-3}$, overlapping with the lower limit listed in Table 5. Moreover, Reale et al. (1988) estimated a loop magnetic field of $\sim 100 \mathrm{G}$ for the 1980 flare, consistent with the HSA lower limit of $73 \mathrm{G}$ in Table 5.

Poletto et al. (1988) also applied their MR model to the 1980 flare on Prox Cen. They derived a field strength of 150-1000 G (consistent with the lower limit in Table 5 for the 1993 flare). The reconnection model cannot distinguish between a large region (extending over $33^{\circ}$ in latitude) of weak field and a small region (extending over $5^{\circ}$ of latitude) of strong field. Combining the observed EM with the assumed latitudinal extent, Poletto et al. (1988) derived $N_{e}=(0.9-10) \times 10^{11} \mathrm{~cm}^{-3}$ at the flare peak. Loop lengths range from $20 \times 10^{9} \mathrm{~cm}\left(\approx R_{*}\right)$ down to $\approx 0.2 R_{*}$. These densities and lengths overlap with the HSA values for the 1993 flare in Table 5 .

The quiescent corona in Prox Cen, detected by Einstein, yields a 2-T fit where $T_{H}=16-20 \mathrm{MK}$ (Schmitt et al. 1990). The HSA estimate of flare $T$ (Table 5) provides only a lower limit on $T$ : if the true $T$ is close to the HSA $T$ limit, then the HSA $T$-value would violate the criterion $T>T_{H}$.

\subsubsection{UV Cet $(=G l 65 B=L 726-8 B)$}

Guedel \& Benz $(2005)^{2}$ report that, with cyclotron emission as the source of the observed quiescent radio flux, the field strength in the radio-emitting region of UV Ceti in nonflaring conditions must be in the range 600-2070 G. In a different type of analysis offlares observed in X-rays and in radio, Smith et al. (2005) use the properties of nonthermal electrons to estimate that the field strengths in flaring loops on UV Cet may be in the range $160-200 \mathrm{G}$.

These results suggest that coronal fields in various loops on UV Ceti may span a range of as much as an order of magnitude. We note that in Table 5 the 15 UV Cet flares that were observed by $E U V E$ also have estimates of the lower limit on $B$ that range over an order of magnitude (or more): the HSA estimates range from less than 100 to almost $1700 \mathrm{G}$. This range of HSA field estimates overlaps well with the ranges reported by Guedel \& Benz (2005) and Smith et al. (2005).

A different class of magnetic structures in UV Cet (Benz et al. 1998) appears to contain fields that are considerably weaker than the fields estimated for the flaring loops in Table 5: fields of 15-130 G are reported. However, the linear scale of these structures is several stellar radii, i.e., much larger than the flaring loops that have emerged from the HSA modeling in Table 5. Thus,

\footnotetext{
${ }^{2}$ Available at http://www.astro.phys.ethz.ch/papers/guedel/uvcet/uvcet.ps.
} 
there is no inconsistency in the fact that the lower limits on fields in Table 5 are in almost all cases considerably stronger than the fields reported by Benz et al. (1998).

In an indirect manner, we would like to propose another method of checking on the reliability of the loop parameters derived by HSA. The original application of HSA to a UV Cet flare (Haisch 1983) indicated that, using the parameters that had been determined, the period of a global mode on the coronal loop that flares in the UV Cet corona would be 60-80 s. Now, if resonant absorption is a viable coronal heating mechanism, then loops should be oscillating in a global mode (e.g., Mullan \& Johnson 1995) and might be expected to show periodicities on the above scales. Variabilities in radio emission and in X-ray emission from UV Cet have in fact been detected with periods of 56 (Gary et al. 1982) and $70 \mathrm{~s}$ (Mullan \& Johnson 1995), respectively. These results suggest that certain combinations of loop parameters derived by HSA are not unrealistic.

Based on 2-T fits to Einstein data, the hot component in the quiescent corona has $T_{H}=16-22 \mathrm{MK}$ (Schmitt et al. 1990). Most of the flares in Table 5 have HSA-derived $T$-values that exceed these values, as the FHC test requires.

\subsubsection{CN Leo $(=$ Gl 406=Wolf 359)}

This star, one of the closest to Earth $(d=2.39 \mathrm{pc})$, is a wellknown optical flare star (Lacy et al. 1976). It is unique, among a sample of $15 \mathrm{M}$ dwarfs, in that CN Leo's quiescent corona is detectable from the ground, using the forbidden Fe XIII line at $3388.1 \AA$ (Fuhrmeister et al. 2004).

$\mathrm{CN}$ Leo is late enough in spectral type that its internal structure may be completely convective (although this may not be the case if magnetic fields impede the onset of convection; Mullan \& MacDonald 2001). If the star is indeed completely convective, then it could be a candidate for the study of dynamo operation in a star where no interface dynamo is possible: the properties of magnetic loops on this star may be determined solely by distributed dynamo processes. In this regard, we note that the flare energy distribution, with a slope of $\beta=-1$ or steeper (Lacy et al. 1976; Gershberg \& Shakhovskaya 1983), is such that small flares dominate the energy budget in CN Leo. (For most flare stars, the largest flares dominate the budget.) Whether or not this dominance of smaller flares extends all the way to the very smallest events ("nanoflares"), so that coronal heating could be explained by flaring, is questionable (Robinson et al. 1995; Audard et al. 2000). But if small-flare dominance is characteristic of a distributed dynamo, then CN Leo may be a prime candidate for dynamo studies.

ROSAT PSPC observations of X-rays from $\mathrm{CN}$ Leo in quiescence have allowed the extraction of reliable 2-T models: these indicate that the cool component of the quiescent corona has a best-fit value of $T_{L}=1.71 \mathrm{MK}$ (Giampapa et al. 1996), among the coolest known coronae of any star. The hot component in $\mathrm{CN}$ Leo has a best-fit temperature of $T_{H}=8.2 \mathrm{MK}$ (Giampapa et al. 1996). This is certainly the coolest hot component in our sample offlare stars. Now, as far as flare energies are concerned, among a sample of 23 flare stars, flares on CN Leo release the smallest amount of flare energy in the $U$ and $B$ bandpasses (Lacy et al. 1976; Gershberg \& Shakhovskaya 1983). In view of this, it is interesting to note that the temperatures derived by the HSA method for flares on CN Leo are lower than for any other star in Table 5. Nevertheless, we note that in none of the CN Leo flares does the flare temperature lie definitely below the $T_{H}$ value derived by Giampapa et al. (1996). That is, the HSA method leads to flare temperatures that are in all 13 cases hotter than the hot component of the quiescent corona, as the FHC test requires.
Giampapa et al. (1996) find (see their Fig. 3f) that hot loops in CN Leo have lengths $L_{H}$ that may be as large as $\sim s_{p}$, where the pressure scale height $s_{p}$ has the value $1.1 \times 10^{10} \mathrm{~cm}$, comparable to $R_{*}$. We see that our estimates of $L / R_{*}$ (Table 5 ) include values that are comparable to 1 .

Micela et al. (1997) performed spectral fits to ROSAT PSPC data during a flare on CN Leo. The hot component of the flare was found to have a temperature in the range 5.8-11.6 MK, with a most likely value of $8.1 \mathrm{MK}$. In order to be consistent with the FHC test, the temperature of the hot flare component should exceed $T_{H}(=8.2 \mathrm{MK})$ in the quiescent corona. This suggests that the hot flare component should have a temperature closer to the upper end of the range derived by Micela et al. (1997). With this adjustment, the flare $T$ derived by Micela et al. (1997) would overlap with many of the HSA values of flare $T$ in Table 5 .

Especially valuable in the present context is the fact that a full $\mathrm{HM}$ of the ROSAT PSPC flare on CN Leo was subsequently performed by Reale \& Micela (1998). Substantial heating was required in the decay phase, so the contrast with the HSA could hardly be more pronounced. Nevertheless, the loop length was found to be $1.4 \times 10^{10} \mathrm{~cm}$, i.e., $1.3 R_{*}$, using a stellar radius of $0.16 R_{\odot}$. [Note that the radius of CN Leo cannot be as large as $0.3 R_{\odot}$ as claimed by Reale \& Micela (1998): spectrophotometry indicates that the radius is $(1.11 \pm 0.1) \times 10^{10} \mathrm{~cm}$ (Leggett et al. 2000), i.e., $R_{*}=0.16 \pm 0.02 R_{\odot}$.] Loops as long as $1.3 R_{*}$ are consistent with the larger loops in Table 5.

Also according to the HM of Reale \& Micela (1998), the maximum temperature in the flare was found to be $7.5 \mathrm{MK}$. Unless this result is subject to errors of $10 \%$ or more, it is difficult to understand how it could be consistent with flare heating: the flare temperature would be less than that of a hot loop in the quiescent corona (Giampapa et al. 1996). We note that 7.5 MK coincides with the lowest end of the temperature range derived by HSA for one of the flares in CN Leo (Table 5): for that flare, the upper end of the HSA temperature range definitely exceeds the $T_{H}$ of the hot quiescent loops, as the $\mathrm{FHC}$ test requires.

Since the values of $T_{H}$ and the associated loop lengths obtained by Giampapa et al. (1996), as well as the flare temperatures obtained by Micela et al. (1997) and the loop lengths derived by Reale \& Micela (1998), were all determined in completely different ways from the approach we have used, we consider these results as a valuable consistency check on the HSA method.

As regards magnetic field strength, the absence of detectable radio emission from $\mathrm{CN}$ Leo in quiescence at wavelengths of 6 and $20 \mathrm{~cm}$ suggests that the magnetic field in the corona cannot exceed a few hundred gauss (O'Dea \& McKinnon 1987). These estimates refer to field strengths at the "radio photosphere," corresponding to heights of at most $2.1 R_{*}-3.2 R_{*}$ above the stellar surface. Closer to the star, at radial distances corresponding to loops listed in Table 5, the upper limits on field strengths would be even stronger than the above limit. These are consistent with the lower limits in Table 5.

\section{REVISITING THE RELIABILITY OF HSA ESTIMATES OF LOOP PARAMETERS}

We wish to determine, for each of our target stars, whether or not the HSA estimates of $L, B, T$, and $N_{e}$ derived here for $E U V E$ flares are consistent with results obtained by other researchers. Our assessments are given in Table 6.

If other workers use a method of analysis that is independent of the HSA, we attach greater weight to their conclusions. If such workers have reported a value of, say, $L$ in a coronal loop for a star that is consistent with the HSA estimates in Tables 4 and 5 for $E U V E$ flares in that star, then a plus sign is entered for 
TABLE 6

Evaluation of Results

\begin{tabular}{|c|c|c|c|c|c|}
\hline Number & Star & $L$ & $B$ & $T$ & $N_{e}$ \\
\hline $1 \ldots \ldots \ldots \ldots$ & HR 120 & 0 & 0 & 0 & 0 \\
\hline $2 \ldots \ldots \ldots \ldots$ & HR 1817 & ++ & ++ & ++ & + \\
\hline $3 \ldots \ldots \ldots \ldots$ & $\sigma^{2} \mathrm{CrB}$ & +- & + & +++ & - \\
\hline $4 \ldots \ldots \ldots \ldots . . .$. & $\chi^{1}$ Ori & + & + & + & + \\
\hline $5 \ldots \ldots \ldots \ldots$ & EK Dra & $(+)$ & $(+)$ & ++ & $(+)$ \\
\hline $6 \ldots \ldots \ldots \ldots . .$. & 44 Boo & ++ & 0 & + & + \\
\hline $7 \ldots \ldots \ldots \ldots$ & DK UMa & + & + & + & 0 \\
\hline $8 \ldots \ldots \ldots$ & $\kappa$ Cet & ++ & + & + & 0 \\
\hline $9 \ldots \ldots \ldots \ldots$ & ER Vul & +++ & 0 & + & + \\
\hline $10 \ldots \ldots \ldots \ldots$ & $\mu \mathrm{Vel}$ & - & 0 & ++ & -+ \\
\hline $11 \ldots \ldots \ldots \ldots$ & $\xi$ Boo & ++- & + & + & + \\
\hline $12 \ldots \ldots \ldots \ldots$ & VW Cep & -+- & ++ & -+-- & -+ \\
\hline $13 \ldots \ldots \ldots \ldots$ & LQ Hya & +- & ++ & - & ++ \\
\hline $14 \ldots \ldots \ldots \ldots$ & $\mathrm{BH} \mathrm{CVn}$ & - & - & 0 & + \\
\hline $15 \ldots \ldots \ldots \ldots$ & AR Lac & + & 0 & + & -+- \\
\hline $16 \ldots \ldots \ldots \ldots$ & UX Ari & +++ & + & ++ & ++ \\
\hline $17 \ldots \ldots \ldots . . .$. & $\beta$ Cet & 0 & + & + & -+ \\
\hline $18 \ldots \ldots \ldots \ldots$ & HD 37394 & 0 & 0 & 0 & 0 \\
\hline $19 \ldots \ldots \ldots . . .$. & V711 Tau & +- & + & ++ & + \\
\hline $20 \ldots \ldots \ldots \ldots$ & AR Psc & $(-)(-)$ & $(+)(+)$ & 0 & $(-)(-)$ \\
\hline $21 \ldots \ldots \ldots \ldots$ & $\sigma \mathrm{Gem}$ & ++ & + & + & - \\
\hline $22 \ldots \ldots \ldots \ldots$ & AB Dor & +++ & + & 0 & + \\
\hline $23 \ldots \ldots \ldots \ldots$ & Gl 117 & 0 & + & 0 & 0 \\
\hline $24 \ldots \ldots \ldots \ldots$ & $\epsilon$ Eri & + & 0 & 0 & 0 \\
\hline $25 \ldots \ldots \ldots \ldots$ & PW And & + & 0 & 0 & 0 \\
\hline $26 \ldots \ldots \ldots \ldots$ & II Peg & $++(+)++(-)-$ & ++ & $++(+)(+)$ & $++(+)+$ \\
\hline $27 \ldots \ldots \ldots \ldots$ & BY Dra & +- & + & + & + \\
\hline $28 \ldots \ldots \ldots \ldots$ & DH Leo & + & 0 & + & 0 \\
\hline $29 \ldots \ldots \ldots \ldots$ & $\xi \mathrm{UMa}$ & +- & + & + & -- \\
\hline $30 \ldots \ldots \ldots \ldots$ & AU Mic & $++(+)$ & - & + & -+ \\
\hline $31 \ldots \ldots \ldots . .$. & CC Eri & +++ & +++ & + & 0 \\
\hline $32 \ldots \ldots \ldots \ldots$ & YY Gem & -+++++ & + & -+++ & + \\
\hline $33 \ldots \ldots \ldots \ldots$ & G1 205 & + & + & 0 & 0 \\
\hline $34 \ldots \ldots \ldots \ldots$ & Gl 411 & 0 & 0 & 0 & 0 \\
\hline $35 \ldots \ldots \ldots \ldots$ & Gl 644 & ++- & ++ & ++ & 0 \\
\hline $36 \ldots \ldots \ldots \ldots$ & FK Aqr & 0 & 0 & + & 0 \\
\hline $37 \ldots \ldots \ldots \ldots$ & EUVE 0613-23.9B & 0 & 0 & 0 & 0 \\
\hline $38 \ldots \ldots \ldots \ldots$ & AD Leo & ++++- & ++ & ++ & ++ \\
\hline $39 \ldots \ldots \ldots \ldots$ & EV Lac & $+(+)-+-$ & ++ & ++++ & $+(+)$ \\
\hline $40 \ldots \ldots \ldots \ldots$ & YZ CMi & + & 0 & $(+)$ & 0 \\
\hline $41 \ldots \ldots \ldots \ldots$ & EQ Peg & $(-)-(+)++$ & ++ & $(-)++++$ & $(+)(+)+$ \\
\hline $42 \ldots \ldots \ldots \ldots$ & Prox Cen & ++ & ++ & $+(-)$ & ++ \\
\hline $43 \ldots \ldots \ldots \ldots$ & UV Cet & 0 & $++(-)$ & + & 0 \\
\hline $44 \ldots \ldots \ldots \ldots$ & CN Leo & ++ & + & +++ & 0 \\
\hline
\end{tabular}

that star in the $L$ column. If the independent method yields a value of, say, $B$ that is inconsistent with results in Tables 4 and 5 , then a minus sign is entered for that star in the $B$ column. We enter one symbol in Table 6 for each independent estimate that we were able to locate in the literature for any loop parameter on a particular star.

If other workers apply a quasi-static approach for their analysis of a flare light curve, then we regard their results as providing less independent support for our conclusions: we enter those results (both + and - ) in parentheses in Table 6 .

In cases where we have found no results for a parameter in the literature that are relevant for a particular star, we enter 0 in Table 6.

Adding up plus signs and minus signs in Table 6, we see that, based only on estimates of loop properties that have been performed independently of the approach we use here, the "score" is 180:32 in support of our results. In Table 6, there are also 53 entries of zeros: in each case, a zero indicates that we were not

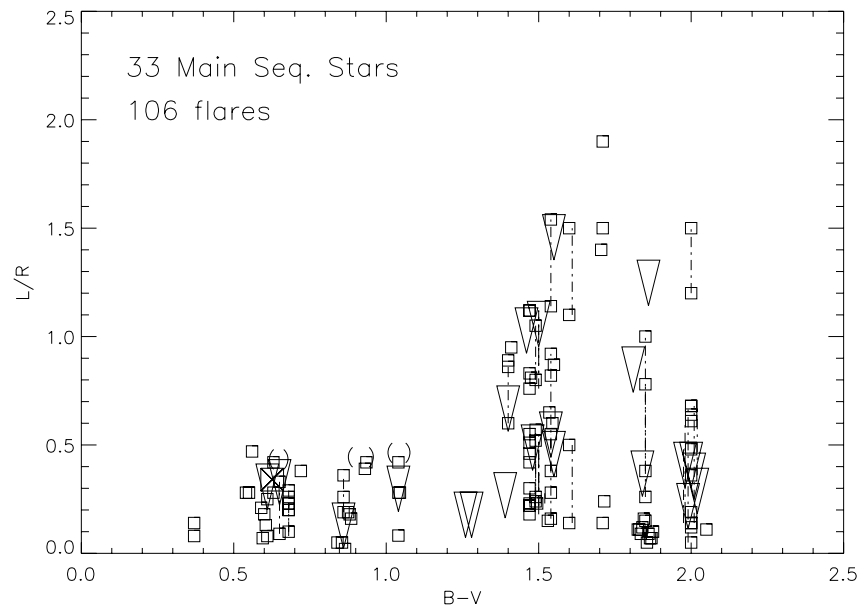

FIG. 1.-Main-sequence stars: ratio of loop length $L$ to stellar radius $R$ as a function of $B-V$ color.

able to find any estimate for that parameter in the literature. In the unlikely event that in the future data become available that have the effect that all of those 49 zeros are replaced by negatives, the score would still be such that positives would predominate over negatives by a ratio of $2: 1$. This gives us confidence that the HSA approach yields loop parameters that are supported by other approaches to the analysis of flaring loops.

\section{DISCUSSION}

Since stars on the main sequence have different internal structures from those in evolved stars, dynamo action may operate in physically different ways in dwarfs and giants. In support of this, we note that there is a difference in the rotation-activity connection for dwarfs and giants in RS CVn systems: among the fastest rotating dwarfs, there is a saturation effect in the strength of the EUV emission, whereas the evolved stars show no such effect (Mitrou et al. 1997). Because of this, we believe that it is important, from the standpoint of dynamo physics, to discuss the results for main-sequence stars and evolved stars separately.

\subsection{Main-Sequence Stars}

The principal result of our study is shown in Figures 1 and 2, which differ only in the color that is used as the abscissa. The HSA-derived $L / R_{*}$ ratios for the main-sequence stars in our sample are plotted as a function of $B-V$ in Figure 1 . Since $B-V$ becomes a poor discriminator of spectral type among the later $\mathrm{M}$ dwarfs, we also plot the HSA-derived $L / R_{*}$ ratios for the main-sequence stars in our sample as a function of $V-I$ in Figure 2 . In the case of stars with multiple flares, for the sake of clarity, each flare is plotted with an individual color that is slightly offset on either side of the star's true color. Upper limits on $L / R_{*}$ are plotted as downward-pointing open triangles. If a particular flare yields a range of $L / R_{*}$ values in a given star, the results are plotted as a vertical line at the $B-V$ value appropriate for that star.

Inspection of Figures 1 and 2 suggests that we may profitably divide the sample of EUVE DS flaring stars into two subsamples: a subsample of cool stars and another subsample of warm stars. The cool and the warm subsamples are somewhat easier to distinguish by eye in Figure 2 (where there is a clear gap in $V-I$ color) than in Figure 1 (where the $B-V$ colors lie closer together). In the cool subsample, with $B-V \geq 1.3-1.4$ and $V-$ $I \geq 2.0$ (containing 18 stars), loop lengths vary from much smaller than $R_{*}$ to almost $2 R_{*}$. In the warm subsample, with $B-V \leq 1.04$ 


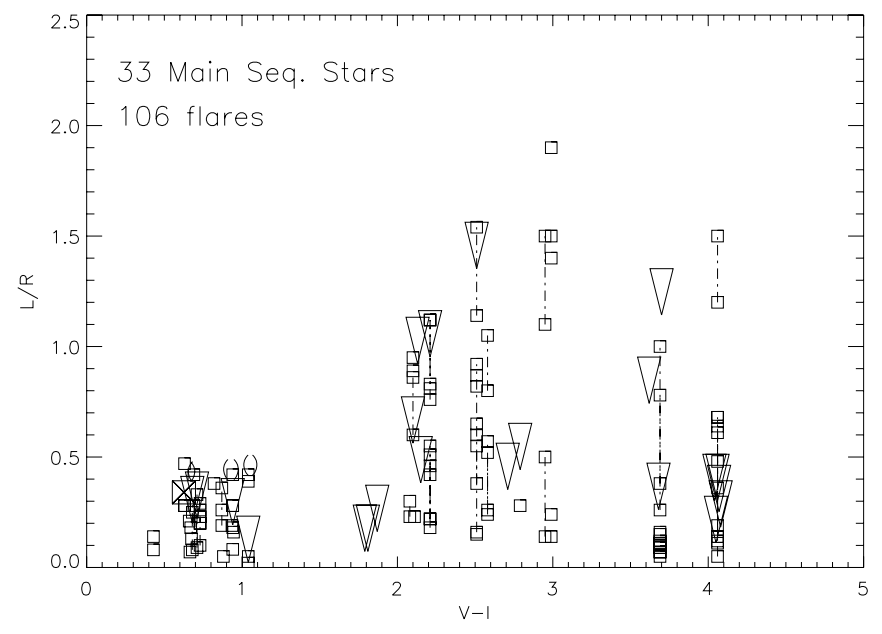

FIG. 2.-Main-sequence stars: ratio of loop length $L$ to stellar radius $R$ as a function of $V-I$ color. In this plot, the M dwarfs are less crowded.

and $V-I \leq 1.1$ (containing 15 stars), loop lengths are confined to values that are less than $0.5 R_{*}$. Thus, the two subsamples (containing comparable numbers of stars) have upper limits in Figures 1 and 2 that differ in magnitude by a factor of 3-4.

Because the transition between stars containing short loops only and stars containing both short and long loops occurs over a relatively narrow range of $B-V$ color in Figure 1, we refer to this transition for brevity as a "step." The amplitude of the step is 3-4.

Although the Sun is (obviously!) not a member of our sample of EUVE flaring stars, we know enough about the Sun to allow us to plot a data point in Figure 1. This is the large square containing an $\mathrm{X}$ in Figure 1: the symbol is at a location corresponding to the "large-scale structures" mentioned by Golub et al. (1980). With half-lengths of $1.2 \times 10^{10} \mathrm{~cm}$ (Golub et al. 1980, their Table 2), these structures correspond to $L / R$ (in our notation) of 0.34 . We plot this value at the color of the Sun: $B-$ $V=0.626$ (Sekiguchi \& Fukugita 2000). The Sun's largest loops do not violate our conclusion about the absence of long flaring loops in warmer stars.

Note also that the two F stars in our sample (HR 120 and HR 1817), rare examples of flare stars at relatively early spectral type, provide valuable information in pinning down the upper envelope on $L / R_{*}$ at the left side of Figure 1.

\subsection{Transition in Loop Sizes: An Artifact of the HSA Method?}

The HSA method of analyzing flare light curves that we have used in the present paper is a simplified approach. Is it possible that some artifact of the HSA method gives rise to the step in loop lengths such as we present in Figure 1? We consider three possibilities here:

1. Differences in the rate offlare energy release. When HM is used to analyze the light curve of a flare in which the release of flare energy is assumed to be instantaneous, the loop length sometimes turns out to be larger than the HSA method indicates (Reale et al. 1988). On the other hand, when one allows for continuous energy release (after flare maximum), HM sometimes leads to loop lengths that are smaller than the HSA method would give (Favata et al. 2001).

In view of these results, it is conceivable that the step in Figure 1 could be an artifact of the HSA method if the following scenario were true: flares on stars in the warm subsample are preferentially events in which the energy release is instantaneous, whereas stars in the cool subsample preferentially have flares where the energy release is continuous. But we see no reason why this alteration in flare behavior should occur in "real stars."

2. Errors in EM. We have seen in our discussion of AD Leo that uncertainties in iron abundance in the flaring plasma could lead to significant errors in our estimates of EM (factor of 10). According to equations (1)-(3), errors in EM propagate into errors in loop length: $L \sim(\mathrm{EM})^{0.25}$. In view of this, we see that in order for errors in EM to account for the step of amplitude 34 in $L$, the EM values that we have used for flares in stars belonging to the warm subsample would have to be erroneously too small by factors of 81-256 compared to the EM values in the cool subsample. Alternatively, the EM values that we have used in our analysis for flares in stars belonging to the cool subsample would have to be erroneously too large by factors of 81256 compared to the EM values in the warm subsample. Now, we have seen that our estimates of EM are subject to uncertainties associated with coronal abundances of iron (see discussion of AD Leo above). This leads us to speculate on an "abundance scenario" as a possible explanation for the step in Figures 1 and 2: suppose that flares on stars in the cool subsample have (for some reason) Fe abundances that are 10 times larger than the solar coronal value, while flares on stars in the warm subsample have (for some reason) Fe abundances that are 10 times smaller than the solar coronal value. In such a scenario, a reanalysis of the observed flares would cause the step in Figures 1 and 2 to disappear. But we can see no reason why such a systematic (and drastic) 100-fold increase in coronal iron abundances should occur in real stars as the spectral type changes from $\mathrm{K} 0$ to $\mathrm{M} 0$. In fact, evidence based on the first ionization potential (FIP) effect suggests that just the opposite is more likely to occur. To see this, note that in the Sun, where activity levels are low, the FIP effect indicates that Fe is enhanced in the corona relative to the photosphere. On the other hand, in stars with high levels of activity, an "inverse FIP effect" has been reported (e.g., Suh et al. 2005): in such stars, no enhancement of Fe occurs in the corona. Since M dwarfs are the most active flare stars known, stars in our cool subsample (all of which are $\mathrm{M}$ dwarfs) should tend to exhibit the inverse FIP effect, with no enhancement in coronal Fe abundance. On the other hand, stars in our warm subsample (i.e., main-sequence stars with spectral types F, G, and K) in general have lower levels of activity. Such stars are more likely to exhibit a solar-like FIP effect, with Fe enhanced in the corona. This is precisely opposite to the systematic change in $\mathrm{Fe}$ abundance that would be required in the abundance scenario that was explored above. Thus, we do not believe that the abundance scenario is a viable means to explain away the step in Figures 1 and 2. In fact, if our reasoning based on the FIP effect is correct, then the amplitude of the step in Figures 1 and 2 may be even larger than we have plotted it.

3 . Is it conceivable that there might be certain temperature distributions in flare plasma that could give rise to pathologically different spectra in the EUVE DS bandpass in different stars? It does not seem likely. After all, a flare involves simply an energy release, whether it occurs on a star in the warm subsample or on a star in the cool subsample: in fact, the correlation that exists between flare temperature and flare EM in solar flares also apparently extends to stellar flares (Feldman et al. 1995). (It was to explain this universal correlation that Shibata \& Yokoyama [1999] developed their reconnection model that we mentioned in $\S 2.1$.) It is hard to see why there should be such enormous differences in the EUV spectra as to cause EM estimates to be systematically in error by a factor of 100 or more in one subsample but not in the other. Moreover, the systematic errors would have to grow rapidly across a narrow range of 
spectral types if this artifact were to be responsible for the step in Figures 1 and 2.

In what follows, we consider that the step in Figures 1 and 2 is real.

\subsection{Location of the Transition to Large Loops on the Main Sequence}

With the limited sample of stars in our sample, it is not easy to define precisely in terms of spectral type where the transition in Figure 1 between the cool subsample and the warm subsample occurs. All we can say is that the stars containing large loops appear to have $B-V \geq 1.4$, corresponding to spectral type $\mathrm{M} 0$ and later, while the stars that do not contain large loops have $B-V \leq 1.0$, corresponding to spectral type $\mathrm{K} 2$ or earlier. The gap between subsamples is easier to see in Figure 2, but the conclusion is the same. The one star in our sample that lies in the gap (BY Dra: K4+K7) does not help us here because the only flares observed on that star yield only upper limits on loop sizes, and those upper limits are small. For future reference, BY Dra might conceivably help to narrow the gap if observers in the future are lucky enough to observe a flare in a large loop on that system. But even then, it might be difficult to assign the flare to one particular component.

The conservative conclusion that we draw from our results is the following. As we consider stars that lie progressively farther down along the main sequence, the transition to large loops might occur as early as $\mathrm{K} 2+$ but does not occur any later than $\mathrm{M} 0-$.

Information that has a bearing on the location of the transition may be available from microwave data. According to an analysis of observations of flare stars at frequencies of 8 and $15 \mathrm{GHz}$, White et al. (1994) conclude that the hot coronal component (which the authors ascribe to flaring plasma) is "likely to be restricted to heights of the order of a stellar radius above the photosphere." In that study, White et al. (1994) observed 19 stars, of which 16 were of spectral type M0 and later: in fact, the title referred to "dMe" stars only. However, their sample also included three K stars, of spectral type K5, K6 Ve, and K7 Ve. Thus, their conclusion about loops extending to heights of order $R_{*}$ above the photosphere might apply to stars as early as K5. If this result can be verified independently, then when combined with our conclusions, the transition to large loops may be narrowed down to the range of spectral types between $\mathrm{K} 2+$ and $\mathrm{K} 5-$.

Although our conclusion about the location of the large loop boundary is not as precise as we would like, it nevertheless has a valuable bearing on dynamo theory. To see why, note that in discussions of stellar structure, the limiting spectral types that are cited for the onset of completely convective stars on the main sequence are no earlier than M3-M4. In fact, when magnetic effects are included in the models, the onset of complete convection may be postponed to spectral types as late as M8 (Mullan \& MacDonald 2001). Both of these limits are significantly later than the spectral type at which we have found the transition between large and small loops in flaring main-sequence stars.

If our derivation of a transition to large loops between $\mathrm{K} 2+$ and $\mathrm{M} 0-($ or $\mathrm{K} 5-)$ is reliable, then the onset of large loops in the coolest main-sequence stars occurs for reasons that are not necessarily connected with the transition to complete convection. This may help to explain why the $\mathrm{X}$-ray properties of $\mathrm{M}$ dwarfs do not exhibit any peculiarities at the (nominal) transition to complete convection (Fleming et al. 1993).

\subsection{Small Loops in the Coolest Main-Sequence Stars}

As well as large loops, there are also many examples of short loops in certain late M stars, especially UV Cet. Formally, the

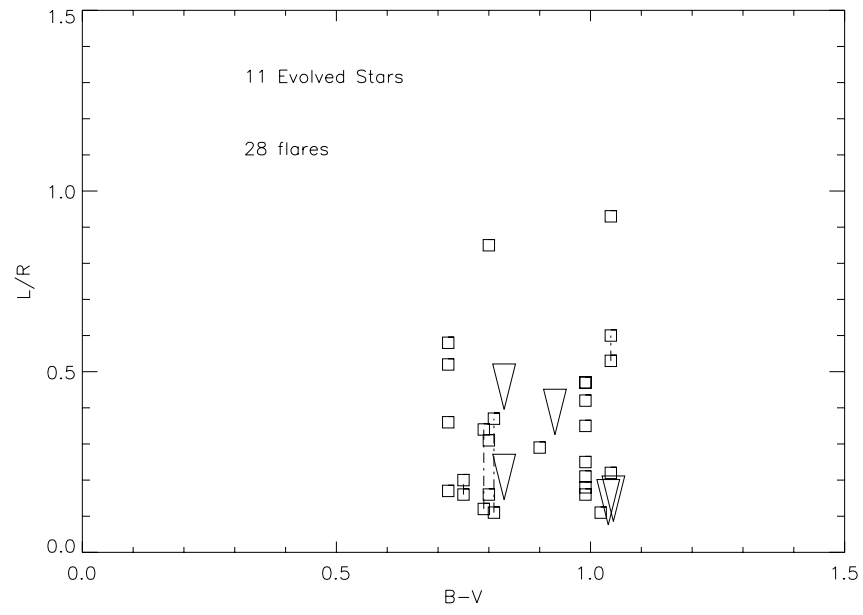

FIG. 3.-Evolved stars: ratio of loop length $L$ to stellar radius $R$ as a function of $B-V$ color.

smallest loop size we have derived $\left(0.05 R_{*}\right.$ in UV Cet) is more than 1 order of magnitude smaller than the largest loop size in the same star. And for CN Leo, there is a formal factor of 30 difference between the lengths of the smallest and the largest flaring loop in our sample. A range of 30 in length scales corresponds roughly to the range of solar magnetic structures that were the subject of power spectrum analysis by Nakagawa \& Levine (1974). This suggests that we have had some success in the undertaking that is described in the last paragraph of $\S 1$ : we have obtained information concerning the distribution of loop sizes in stars even in the absence of images.

\subsection{Angular Momentum Loss in Dwarf Stars}

Finally, we note that large magnetic loops in a stellar corona impede the loss of mass, whereas small loops favor mass loss (e.g., Mullan \& MacDonald 2003). If indeed the stars of lowest mass are sites of the largest loops, as Figure 1 suggests, the mass loss from such stars may be impeded enough to explain the lack of angular momentum loss as they evolve (Giampapa et al. 1996).

\subsection{Evolved Stars}

In Figure 3 we show the values of $L / R_{*}$ as a function of $B-V$. Small and large loops are present in the target stars. There seems to be little evidence for a trend in loop lengths as a function of $B-V$. As a result, we do not see how our results can shed significant light on dynamo processes in evolved stars.

\section{CONCLUSIONS}

We have applied a uniform analysis to a strictly homogeneous set of light curves of 134 flares obtained by the same instrument (EUVE DS). We have tried to ensure that our sample of 44 flaring stars is reasonably complete: on surveying the entire EUVE DS database, we believe that our sample includes the great majority of stars that were observed to have at least one analyzable flare in EUVE DS. Our aim has been to determine in a uniform manner the parameters of magnetic loops in as broad a sample of cool flaring stars as possible. As it is, our sample includes stars with spectral types extending from F2 to M6. We have shown that the HSA method yields parameters for flaring loops that are consistent with estimates made by a variety of other methods.

We have found that among main-sequence stars, our targets fall into two subsamples, with roughly equal populations. In the cool subsample (18 stars), we find that large loops (with lengths up to almost $2 R_{*}$ ) exist on stars with spectral type M0 and later. In the 
warm subsample (15 stars plus the Sun), with spectral type K2 and earlier, we find that loop lengths are no larger than $0.5 R_{*}$. Our data suggest that there is a transition between short and long loops on the main sequence between spectral types K2 and M0. Certain microwave observations (White et al. 1994) suggest that long loops may be present on stars as early as K5. If the microwave loops are related to the EUVE flaring loops, then the transition between short and long loops may occur between K2 and $\mathrm{K} 5$.

Is there a physical reason why there might be a preference for loop lengths of $\leq 0.5 R_{*}$ in the warm subsample of flaring stars? Work by Schrijver $\&$ Title (2005) points to a possible reason. Schrijver \& Title (2005) have numerically modeled the surface distribution of fields in solar-like stars. (The stars in our warm subsample have effective temperatures that are solar-like.) In their model, the field structures that build up on the surface of a solarlike star are determined by the injection of flux into the photosphere, followed by various physical processes (random walk, differential rotation, and meridional flow) that redistribute the flux over the surface. Stars with different activity levels are modeled by varying the flux emergence rate, from 1 to 30 times the solar value. Even with this large range of activity levels, the surface fields on solar-like stars continue to exhibit the pattern that is characteristic of solar active regions: the fields appear as a large number of bipoles, with most bipoles confined to two relatively narrow bands of latitudes north and south of the equator. This has a significant consequence when one calculates the distribution of loop lengths: when the star is at an activity maximum, there is a peak in the distribution at a few tenths of a solar radius. Schrijver \& Title (2005) state, "loops with lengths between 0.04 and $0.4 R_{\odot}$. . dominate the loop length spectrum." The average length of closed coronal field lines in solar-like stars at activity maximum is $0.4 R_{\odot}$ (see Fig. 3 of Schrijver \& Title 2005). When the star is at the minimum of its activity cycle, the results are somewhat different; during that phase, the polar coronal holes dominate the magnetic structure, and longer loops $\left(1-2 R_{\odot}\right)$ appear in significant numbers in the distribution. However, observational selection is at work in our EUVE study: we have selected only those stars where flares were in fact detected. This suggests that our sample is biased toward stars that are near maximum levels of activity. Such stars, if they behave in the way that Schrijver \& Title (2005) have modeled, should have loop lengths that are preferentially a few tenths of $R_{*}$.

For stars in the cool subsample, our results suggests that the Schrijver-Title modeling may not be immediately applicable. In the cool stars, we find that high levels of activity (i.e., flares) occur not only on short loops $\left(L / R_{*} \ll 1\right)$ but also on loops that are long $\left(L / R_{*}=1-2\right)$.

We speculate that the distinction between the stars that fall into our warm and cool subsamples may be due to a transition between two distinct modes of dynamo, perhaps an interface dynamo and a turbulent dynamo distributed throughout the convective envelope. (For arguments that support a turbulent dynamo in the most active dwarfs, see Kashyap \& Drake 1999.) If our speculation is correct, then the transition to a turbulent dynamo occurs between spectral types K2 and M0 (or K2 and K5). This suggests that the transition to a turbulent dynamo does not occur on the main sequence at the transition to complete convection (which occurs at M3-M4, or possibly as late as M8; Mullan \& MacDonald 2001). Instead, the transition may occur in mid-K stars, where the interface between convective envelope and radiative core lies at a depth of about $0.5 R_{*}$. In such a star the star is by no means completely convective.

Is there any evidence that a turbulent dynamo is at work in any of the stars in the cool subsample in Figure 1? We do not know of any at the moment. However, one of the stars in the warm subsample (LQ Hya) is relevant in this context. Donati (1999) has found that the radial and azimuthal field components in LQ Hya are comparable in strength. This is very different from the solar case, where the poloidal (radial) component is orders of magnitude weaker than the toroidal (azimuthal) component. Donati argues that the equality of poloidal and toroidal fields provides evidence for a turbulent dynamo on LQ Hya, a claim that is supported by dynamo modeling (Kitchatinov et al. 2000). Now, a feature to which we wish to draw attention in this context in Figure 1 is the following: among the warm subsample, LQ Hya, EK Dra, and PW And (all young stars with fast rotation) exhibit some of the largest loops of any of the stars in that subsample. (The largest loops in these three stars are highlighted in Fig. 1 by parentheses.) In fact, for LQ Hya, loops that are even larger than we have derived here from EUVE data have been reported by Covino et al. (2001) using a very different analysis from the one we use here. Thus, the only star (LQ Hya) in the warm subsample that is known (empirically) to be the site of a turbulent dynamo happens to be one of the stars in the warm subsample with the longest loops. (Another example may be provided by one of our evolved stars: UX Ari is known to have a large poloidal loop and also a large equatorial loop [Franciosini et al. 1999]. If these loops have equally strong fields, then they may provide evidence for a distributed dynamo in a $\mathrm{K}$ subgiant. However, we do not wish to discuss evolved stars here: we confine attention to main-sequence stars.) The magnetic properties of LQ Hya are consistent with our speculation that the transition to long loops among the coolest stars in Figure 1 may be associated with the onset of a turbulent dynamo.

Is there any theoretical reason to support the possibility that a distributed dynamo might generate large loops in a star? At first, the answer did not seem promising. Durney et al. (1993) suggested that the absence of a radiative core in a low-mass star would "preclude the generation of a large-scale magnetic field." Instead, the turbulent dynamo was expected to generate "small-scale fields." In view of these results, it was not obvious why large loops should occur among late M dwarfs. Recently, however, a detailed numerical model of a turbulent dynamo in a fully convective star has been reported (Dobler et al. 2006). The results are quite different from those of Durney et al. (1993). Dobler et al. (2006) find that a large-scale magnetic field emerges, with loops that span a wide range of length scales. In particular, some loops are quasi-global in extent, spanning many tens of degrees in latitude. Loops that extend, e.g., $50^{\circ}-100^{\circ}$ have $L / R_{*}=1-2$, consistent with the largest loops in Figure 1. To be sure, not all of the stars with large loops in Figure 1 are likely to be completely convective. Dobler et al. (2006) do not indicate how their results would be altered if a (small) radiative core were included in their model. If we are correct in our interpretation of Figure 1, and a distributed dynamo does set in when the radiative core has a radius of $0.5 R_{*}$, then we predict that Dobler et al. (2006) would find essentially the same results at the surface if they replaced their completely convective model with one where a nonconvective core occupies the inner $\frac{1}{8}$ of the stellar volume, i.e., convection occupies "only" $\frac{7}{8}$ of the star's volume.

We are grateful to an anonymous referee for constructive comments. D. J. M. acknowledges financial support from the Particle Physics and Astronomy Research Council (PPARC) while visiting QUB. This research was partially supported by NASA grant NNG04GC75G. 


\section{REFERENCES}

Abada-Simon, M., Lecacheux, A., Aubier, M., \& Bookbinder, J. A. 1997, A\&A, 321, 841

Agrawal, P. C. 1988, A\&A, 204, 235

Agrawal, P. C., Markert, T. H., \& Riegler, G. R. 1985, MNRAS, 213, 761

Agrawal, P. C., Rao, A. R., \& Riegler, G. R. 1986, MNRAS, 219, 777

Agrawal, P. C., White, N. E., \& Riegler, G. R. 1981, MNRAS, 196, 73P

Alef, W., Benz, A. O., \& Guedel, M. 1997, A\&A, 317, 707

Amado, P. J., Doyle, J. G., Byrne, P. B., Cutispoto, G., Kilkenny, D., Mathioudakis, M., \& Neff, J. E. 2000, A\&A, 359, 159

Ambruster, C. W., Fekel, F. C., \& Brown, A. 2003, in The Future of Cool-Star Astrophysics: 12th Cambridge Workshop on Cool Stars, Stellar Systems, and the Sun, ed. A. Brown, G. M. Harper, \& T. R. Ayres (Boulder: Univ. Colorado), 912

Ambruster, C. W., Snyder, W. A., \& Wood, K. S. 1984, ApJ, 284, 270

Andretta, V., \& Giampapa, M. 1995, ApJ, 439, 405

Aschwanden, M. 2004, Physics of the Solar Corona: An Introduction (Berlin: Springer)

Audard, M., Güdel, M., Drake, J. J., \& Kashyap, V. L. 2000, ApJ, 541, 396

Audard, M., Güdel, M., \& Mewe, R. 2001, A\&A, 365, L318

Ayres, T. R., Osten, R. A., \& Brown, A. 1999, ApJ, 526, 445 2001, ApJ, 562, L83

Baliunas, S. L., \& Raymond, J. C. 1984, ApJ, 282, 728

Baliunas, S. L., et al. 1995, ApJ, 438, 269

Barden, S. C. 1984 , AJ, 89, 683

1985, ApJ, 295, 162

Basri, G., \& Marcy, G. W. 1988, ApJ, 330, 274

Benz, A. O., Alef, W., \& Guedel, M. 1995, A\&A, 298, 187

Benz, A. O., Conway, J., \& Guedel, M. 1998, A\&A, 331, 596

Bercik, D. J., Fisher, G. H., Johns-Krull, C. M., \& Abbett, W. P. 2005, ApJ, 631,529

Berdyugina, S. V., Jankov, S., Ilyin, I., Tuominen, I., \& Fekel, F. C. 1998, A\&A, 334, 863

Bradstreet, D. H., Dewarf, L. E., Guinan, E. F., Etzel, P. B., \& Olah, K. 1993, BAAS, 25, 1425

Brickhouse, N., \& Dupree, A. K. 1998, ApJ, 502, 918

Budding, E., Carter, B. D., Mengel, M. W., Slee, O. B., \& Donati, J.-F. 2002, Publ. Astron. Soc. Australia, 19, 527

Budding, E., \& Zeilik, M. 1987, ApJ, 319, 827

Cayrel de Strobel, G., Cayrel, R., Friel, E., Zahn, J.-P., \& Bentolila, C. 1994, A\&A, 291, 505

Choi, C. S., \& Dotani, T. 1998, ApJ, 492, 761

Christian, D. J., Craig, N., Cahill, W., Roberts, B., \& Malina, R. F. 1999, AJ, 117,2466

Christian, D. J., Mathioudakis, M., Jevremovic, D., Dupuis, J., Vennes, S., \& Kawka, A. 2003, ApJ, 593, L105

Contadakis, M. E. 1995, A\&A, 300, 819

Covino, S., Panzera, M. R., Tagliaferri, G., \& Pallavicini, R. 2001, A\&A, 371, 973

Cruddace, R. G., \& Dupree, A. K. 1984, ApJ, 277, 263

Cully, S. L., Fisher, G. H., Abbott, M. J., \& Siegmund, O. H. W. 1994, ApJ, 435, 449

Cully, S. L., Fisher, G. H., Hawley, S. L., \& Simon, T. 1997, ApJ, 491, 910

Cully, S. L., Siegmund, O. H. W., Vedder, P. W., \& Vallerga, J. V. 1993, ApJ, 414, L49

Dempsey, R. C., Linsky, J. L., Fleming, T. A., \& Schmitt, J. H. M. M. 1997, ApJ, 478, 358

Dempsey, R. C., Linsky, J. L., Schmitt, J. H. M. M., \& Fleming, T. A. 1993, ApJ, 413, 333

Dobler, W., Stix, M., \& Brandenburg, A. 2006, ApJ, 638, 336

Donati, J.-F. 1999, MNRAS, 302, 457

Donati, J.-F., Babel, J., Harries, T. J., Howarth, I. D., Petit, P., \& Semel, M. 2002, MNRAS, 333, 55

Doyle, J. G., Butler, C. J., van den Oord, G. H. J., \& Kiang, T. 1990, A\&A, 232,83

Doyle, J. G., \& Mathioudakis, M. 1990, A\&A, 227, 130

Doyle, J. G., van den Oord, G. H. J., \& Kellett, B. J. 1992, A\&A, 262, 533

Drake, J. J., Peres, G., Orlando, S., Laming, J. M., \& Maggio, A. 2000, ApJ, 545,1074

Drake, S. A., Simon, T., \& Linsky, J. L. 1992, ApJS, 82, 311

Drake, S. A., Singh, K. P., White, N. E., \& Simon, T. 1994, ApJ, 436, L87

Duemmler, R., Doucet, C., Formanek, F., Ilyin, I., \& Tuominen, I. 2003, A\&A, 402,745

Durney, B. R., De Young, T., \& Roxburgh, I. 1993, Sol. Phys., 145, 207

Favata, F., Micela, G., \& Reale, F. 2001, A\&A, 375, 485

Fekel, F. 1996, AJ, 112, 269

Feldman, U., Laming, J. M., \& Doschek, G. A. 1995, ApJ, 451, L79
Feldman, U., Landi, E., Doschek, G. A., Dammasch, I., \& Curdt, W. 2003, ApJ, 593,1226

Fleming, T. A., Giampapa, M. S., Schmitt, J. H. M. M., \& Bookbinder, J. A. 1993, ApJ, 410, 387

Franciosini, E., Massi, M., Paredes, J. M., \& Estalella, R. 1999, A\&A, 341, 595 Franciosini, E., Pallavicini, R., \& Tagliaferri, G. 2001, A\&A, 375, 196 Fuhrmeister, B., Schmitt, J., \& Wichmann, R. 2004, A\&A, 417, 701

Gaidos, E. J., Henry, G. W., \& Henry, S. M. 2000, AJ, 120, 1006

Garcia-Sanchez, J., Paredes, J. M., \& Ribó, M. 2003, A\&A, 403, 613

Gary, D. E., Dulk, G. W., \& Linsky, J. L. 1982, ApJ, 263, L79

Gershberg, R. E. 2002, in Solar-like Activity in Main-Sequence Stars (Odessa: Astroprint), 652

Gershberg, R. E., Katsova, M. M., Lovkaya, M. N., Terebizh, A. V., \& Shakhovskaya, N. I. 1999, A\&AS, 139, 555

Gershberg, R. E., \& Shakhovskaya, N. I. 1983, Ap\&SS, 95, 235

Giampapa, M. S., Golub, L., Peres, G., Serio, S., \& Vaiana, G. S. 1985, ApJ, 289,203

Giampapa, M. S., \& Rosner, R. 1984, ApJ, 286, L19

Giampapa, M. S., Rosner, R., Kashyap, V., Fleming, T. A., Schmitt, J. H. M. M., \& Bookbinder, J. A. 1996, ApJ, 463, 707

Golub, L., Maxson, C., Rosner, R., Vaiana, G. S., \& Serio, S. 1980, ApJ, 238, 343

Gondoin, P. 2004a, A\&A, 415, 1113 2004b, A\&A, 426, 1035

Gotthelf, E. V., Jalota, L., Mukai, K., \& White, N. E. 1994, ApJ, 436, L91

Griffin, R. F. 1998, Observatory, 118, 273

Griffiths, N. W. 1999, ApJ, 518, 873

Griffiths, N. W., \& Jordan, C. 1998, ApJ, 497, 883

Guedel, M. 1992, A\&A, 264, L31

Guedel, M., Audard, M., Magee, H., Franciosini, E., Grosso, N., Cordova, F. A., Pallavicini, R., \& Mewe, R. 2001, A\&A, 365, L344

Guedel, M., \& Benz, A. O. 2005, Broad-Band Spectrum of dMe Star Radio Emission, http:www.astro.phys.ethz.ch/papers/guedel/ucvet/ucvet.ps Guedel, M., Guinan, E., \& Skinner, S. 1997, ApJ, 483, 947

Guedel, M., Linsky, J. L., Brown, A., \& Nagase, F. 1999, ApJ, 511, 405

Guedel, M., Schmitt, J. H. M. M., Benz, A. O., \& Elias, N. M., II 1995, A\&A, 301, 201

Haisch, B. M. 1983, in IAU Colloq. 71, Activity in Red-Dwarf Stars, ed. P. B. Byrne \& M. Rodono (Dordrecht: Reidel), 255

Haisch, B. M., Butler, C. J., Doyle, J. G., \& Rodono, M. 1987, A\&A, 181, 96 Haisch, B. M., Drake, J. J., \& Schmitt, J. H. M. M. 1994, ApJ, 421, L39

Hawley, S. L., et al. 1995, ApJ, 453, 464

Heintz, W. D. 1993, PASP, 105, 586

Hempelmann, A., Schmitt, J., \& Stepien, K. 1996, A\&A, 305, 284

Henry, G. W., Fekel, F., \& Hall, D. S. 1995, AJ, 110, 2926

Henry, G. W., \& Newsom, M. S. 1996, PASP, 108, 242

Hill, G. 1989, A\&A, 218, 141

Hooten, J. T., \& Hall, D. S. 1990, ApJS, 74, 225

Huenemoerder, D. P., Canizares, C. R., Drake, J. J., \& Sanz-Forcada, J. 2003, ApJ, 595, 1131

Huenemoerder, D. P., Canizares, C. R., \& Schulz, N. S. 2001, ApJ, 559, 1135

Huovelin, J., Linnaluoto, S., Piirola, V., Tuominen, I., \& Virtanen, H. 1985, A\&A, 152, 357

Hussain, G. A. J., et al. 2005, ApJ, 621, 999

Jackson, P. D., Kundu, M. R., \& White, S. M. 1989, A\&A, 210, 284

Jardine, M., Wood, K., Collier Cameron, A., Donati, J.-F., \& Mackay, D. H. 2002, MNRAS, 336, 1364

Johns-Krull, C. M., \& Valenti, J. A. 1996, ApJ, 459, L95

Kashyap, V. L., \& Drake, J. J. 1999, ApJ, 524, 988

Kashyap, V. L., Drake, J. J., Güdel, M., \& Audard, M. 2002, ApJ, 580, 1118

Kaszas, G., Vinko, J., Szatmary, K., Hegedus, T., Gal, J., Kiss, L. L., \& Borkovits, T. 1998, A\&A, 331, 231

Katsova, M. M., \& Tsikoudi, V. 1993, ApJ, 402, L9

Kitchatinov, L. L., Jardine, M., \& Donati, J.-F. 2000, MNRAS, 318, 1171

Kovari, Zs., Strassmeier, K. G., Bartus, J., Washuettl, A., Weber, M., \& Rice, J. B. 2001, A\&A, 373, 199

Kovari, Zs., Strassmeier, K. G., Granzer, T., Weber, M., Oláh, K., \& Rice, J. B. 2004, A\&A, 417, 1047

Kuijpers, J., \& van der Hulst, J. M. 1985, A\&A, 149, 343

Kundu, M. R., Jackson, P. D., White, S. M., \& Melozzi, M. 1987, ApJ, 312, 822

Lacy, C. H. 1977, ApJS, 34, 479

Lacy, C. H., Moffett, T. J., \& Evans, D. S. 1976, ApJS, 30, 85

Landi, E., Landini, M., \& del Zanaa, G. 1997, A\&A, 324, 1027

Leggett, S. K., Allard, F., Dahn, C., Hauschildt, P. H., Kerr, T. H., \& Rayner, J. 2000, ApJ, 535, 965 
Leto, G., Pagano, I., Linsky, J. L., Rodonò, M., \& Umana, G. 2000, A\&A, 359, 1035

Linsky, J. L., \& Gary, D. E. 1983, ApJ, 274, 776

Little-Marenin, I., Simon, T., Ayres, T. R., Cohen, N. L., Feldman, P. A., Linsky, J. L., Little, S. J., \& Lyons, R. 1986, ApJ, 303, 780

Lopez-Santiago, J., Montes, D., Fernández-Figueroa, M. J., \& Ramsey, L. W. 2003, A\&A, 411, 489

Maggio, A., Favata, F., Peres, G., \& Sciortino, S. 1998, A\&A, 330, 139

Maggio, A., \& Ness, J.-U. 2005, ApJ, 622, L57

Maggio, A., Pallavicini, R., Reale, F., \& Tagliaferri, G. 2000, A\&A, 356, 627

Majer, P., Schmitt, J. H. M. M., Golub, L., Harnden, F. R., Jr., \& Rosner, R. 1986, ApJ, 300, 360

Marcy, G. 1984, ApJ, 276, 286

Marcy, G., \& Bruning, D. H. 1984, ApJ, 281, 286

Marino, A., Micela, G., Peres, G., \& Sciortino, S. 2002, A\&A, 383, 210

Mason, B. D., McAlister, H. A., Hartkopf, W. I., \& Shara, M. M. 1995, AJ, 109,332

Mathioudakis, M., \& Mullan, D. J. 1999, A\&A, 342, 524 (MM99)

Mathioudakis, M., Seiradakis, J. H., Williams, D. R., Avgoloupis, S., Bloomfield,

D. S., \& McAteer, R. T. J. 2003, A\&A, 403, 1101

McIvor, T., et al. 2004, MNRAS, 355, 1066

Messina, S., \& Guinan, E. F. 2002, A\&A, 393, 225

Mewe, R., Kaastra, J. S., van den Oord, G. H. J., Vink, J., \& Tawara, Y. 1997, A\&A, 320, 147

Micela, G., Pye, J., \& Sciortino, S. 1997, A\&A, 320, 865

Mitrou, C. K., Mathioudakis, M., Doyle, J. G., \& Antonopoulou, E. 1997, A\&A, 317, 776

Monsignori Fossi, B. C., Landini, M., Fruscione, A., \& Dupuis, J. 1995, ApJ, 449,376

Montes, D., Fernandez-Figueroa, M. J., de Castro, E., \& Cornide, M. 1995, A\&A, 294, 165

Montes, D., López-Santiago, J., Gálvez, M. C., Fernández-Figueroa, M. J., De Castro, E., \& Cornide, M. 2001, MNRAS, 328, 45

Montesinos, B., Fernandez-Figueroa, M. J., \& de Castro, E. 1987, MNRAS, 229,627

Montesinos, B., \& Jordan, C. 1993, MNRAS, 264, 900

Mullan, D. J., \& Bell, R. A. 1976, ApJ, 204, 818

Mullan, D. J., \& Johnson, M. 1995, ApJ, 444, 350

Mullan, D. J., \& MacDonald, J. 2001, ApJ, 559, 353 2003, ApJ, 591, 1203

Mullan, D. J., \& Mathioudakis, M. 2000, ApJ, 544, 475

Nakagawa, Y., \& Levine, R. H. 1974, ApJ, 190, 441

Ness, J.-U., Güdel, M., Schmitt, J. H. M. M., Audard, M., \& Telleschi, A. 2004, A\&A, 427, 667

Ness, J.-U., Schmitt, J. H. M. M., Burwitz, V., Mewe, R., Raassen, A. J. J., van der Meer, R. L. J., Predehl, P., \& Brinkman, A. C. 2002, A\&A, 394, 911

Newmark, J. S., Buzasi, D. L., Huenemoerder, D. P., Ramsey, L. W., Barden, S. C., Nations, H. L., \& Seeds, M. A. 1990, AJ, 100, 560

O'Dea, C. P., \& McKinnon, W. B. 1987, PASP, 99, 1039

Osten, R. A., \& Brown, A. 1999, ApJ, 515, 746

Osten, R. A., Brown, A., Wood, B. E., \& Brady, P. 2002, ApJS, 138, 99

Osten, R. A., Hawley, S. L., Allred, J. C., Johns-Krull, C. M., \& Roark, C. 2005, ApJ, 621, 398

Osten, R. A., et al. 2004, ApJS, 153, 317

Ottmann, R. 1993, A\&A, 273, 546

Pagano, I., Rodonò, M., Linsky, J. L., Neff, J. E., Walter, F. M., Kovári, Zs., \& Matthews, L. D. 2001, A\&A, 365, 128

Pallavicini, R., Monsignori-Fossi, B. C., Landini, M., \& Schmitt, J. H. M. M. 1988, A\&A, 191, 109

Pallavicini, R., Tagliaferri, G., \& Stella, L. 1990, A\&A, 228, 403

Pallavicini, R., Willson, R. F., \& Lang, K. R. 1985, A\&A, 149, 95

Pan, H. C., \& Jordan, C. 1995, MNRAS, 272, 11

Panagi, P., \& Mathioudakis, M. 1993, A\&AS, 100, 343

Pasinetti Fracassini, L. E., Pastori, L., Covino, S., \& Pozzi, A. 2001, A\&A, 367,521

Pasquini, L., Schmitt, J., \& Pallavicini, R. 1989, A\&A, 226, 225

Perrin, M.-N., \& Karoji, H. 1987, A\&A, 172, 235

Pestalozzi, M. R., Benz, A. O., Conway, J. E., \& Guedel, M. 2000, A\&A, 353, 569

Pevtsov, A. A., Fisher, G. H., Acton, L. W., Longcope, D. W., Johns-Krull, C. M., Kankelborg, C. C., \& Metcalf, T. R. 2003, ApJ, 598, 1387

Poletto, G., Pallavicini, R., \& Kopp, R. A. 1988, A\&A, 201, 93

Ransom, R., Bartel, N., Bietenholz, M. F., Ratner, M. I., Lebach, D. E., Shapiro, I. I., \& Lestrade, J.-F. 2003, ApJ, 587, 390

Reale, F., Betta, R., Peres, G., Serio, S., \& McTiernan, J. 1997, A\&A, 325, 782

Reale, F., \& Micela, G. 1998, A\&A, 334, 1028

Reale, F., Peres, G., Serio, S., Rosner, R., \& Schmitt, J. H. M. M. 1988, ApJ, 328,256
Reid, I. N., Hawley, S. L., \& Gizis, J. E. 1995, AJ, 110, 1838

Ribas, I., Guinan, E. F., Güdel, M., \& Audard, M. 2005, ApJ, 622, 680

Robinson, R. D., Carpenter, K. G., Percival, J. W., \& Bookbinder, J. A. 1995, ApJ, 451, 795

Robrade, J., Ness, J.-U., \& Schmitt, J. 2004, A\&A, 413, 317

Robrade, J., \& Schmitt, J. H. M. M. 2005, A\&A, 435, 1073

Rodono, M. 1978, A\&A, 66, 175

Rodono, M., Pagano, I., Leto, G., Walter, F., Catalano, S., Cutispoto, G., \& Umana, G. 1999, A\&A, 346, 811

Rucinski, S. M. 1992, PASP, 104, 1177

Saar, S. H. 1990, in IAU Symp. 138, The Solar Photosphere: Structure, Convection, and Magnetic Fields, ed. J. O. Stenflo (Dordrecht: Kluwer), 427

Sanz-Forcada, J., Brickhouse, N. S., \& Dupree, A. K. 2002, ApJ, 570, 799

2003, ApJS, 145, 147

Sanz-Forcada, J., \& Micela, G. 2002, A\&A, 394, 653

Scelsi, L., Maggio, A., Peres, G., \& Pallavicini, R. 2005, A\&A, 432, 671

Schaefer, B. E., King, J. R., \& Deliyannis, C. P. 2000, ApJ, 529, 1026

Schmitt, J. H. M. M. 1994, ApJS, 90, 735

Schmitt, J. H. M. M., Collura, A., Sciortino, S., Vaiana, G. S., Harnden, F. R., Jr., \& Rosner, R. 1990, ApJ, 365, 704

Schmitt, J. H. M. M., Fink, H., \& Harnden, F. R. 1987a, ApJ, 322, 1023

Schmitt, J. H. M. M., Pallavicini, R., Monsignori-Fossi, B. C., \& Harnden, F. R., Jr. 1987b, A\&A, 179, 193

Schrijver, C. J., \& Harvey, K. L. 1994, Sol. Phys., 150, 1

Schrijver, C. J., Mewe, R., van den Oord, G. H. J., \& Kaastra, J. S. 1995, A\&A, 302,438

Schrijver, C. J., Mewe, R., \& Walter, F. M. 1984, A\&A, 138, 258

Schrijver, C. J., \& Title, A. M. 2005, ApJ, 619, 1077

Sciortino, S., Maggio, A., Favata, F., \& Orlando, S. 1999, A\&A, 342, 502

Segransan, D., Kervella, P., Forveille, T., \& Queloz, D. 2003, A\&A, 397, L5 Sekiguchi, M., \& Fukugita, M. 2000, AJ, 120, 1072

Shi, J. R., Zhao, G., Zhao, Y.-H., \& You, J.-H. 1998, A\&A, 339, 840

Shibata, K., \& Yokoyama, T. 1999, ApJ, 526, L49 2002, ApJ, 577, 422

Shorlin, S. L. S., Wade, G. A., Donati, J.-F., Landstreet, J. D., Petit, P., Sigut, T. A. A., \& Strasser, S. 2002, A\&A, 392, 637

Siarkowski, M., Pres, P., Drake, S. A., White, N. E., \& Singh, K. P. 1996, ApJ, 473, 470

Singh, K. P., Drake, S. A., Gotthelf, E. V., \& White, N. E. 1999, ApJ, 512, 874

Singh, K. P., Slijkhuis, S., Westergaard, N. J., Schnopper, H. W., Elgaroy, O., Engvold, O., \& Joras, P. 1987, MNRAS, 224, 481

Singh, K. P., White, N. E., \& Drake, S. A. 1996, ApJ, 456, 766

Sirk, M. M., Vallerga, J. V., Finley, D. S., Jelinsky, P., \& Malina, R. F. 1997, ApJS, 110, 347

Skinner, S. L., \& Walter, F. M. 1998, ApJ, 509, 761

Smith, K., Guedel, M., \& Audard, M. 2005, A\&A, 436, 241

Soderhjelm, S. 1999, A\&A, 341, 121

Solanki, S., \& Unruh, Y. 2004, MNRAS, 348, 307

Steltzer, B., et al. 2002, A\&A, 392, 585

Stern, R. A., et al. 1992, ApJ, 391, 760

Strassmeier, K. G., \& Rice, J. B. 2003, A\&A, 399, 315

Suh, J. A., Audard, M., Güdel, M., \& Paerels, F. B. S. 2005, ApJ, 630, 1074

Swank, J. H., \& Johnson, H. M. 1982, ApJ, 259, L67

Tagliaferri, G., White, N. E., Doyle, J. G., Culhane, J. L., Hassall, B. J. M., \& Swank, J. H. 1991, A\&A, 251, 161

Testa, P., Drake, J. J., \& Peres, G. 2004a, ApJ, 617, 508

Testa, P., Drake, J. J., Peres, G., \& DeLuca, E. E. 2004b, ApJ, 609, L79

Toner, C. G., \& Gray, D. F. 1988, ApJ, 334, 1008

Topka, K., \& Marsh, K. A. 1982, ApJ, 254, 641

Trigilio, C., Leto, P., \& Umana, G. 1998, A\&A, 330, 1060

Tsuboi, Y., Imanishi, K., Koyama, K., Grosso, N., \& Montmerle, T. 2000, ApJ, 532,1089

Tsuru, T., Makashima, K., Ohashi, T., Sakao, T., Pye, J. P., Williams, O. R., Barstow, M. A., \& Takano, S. 1992, MNRAS, 255, 192

van den Oord, G. H. J., \& Mewe, R. 1989, A\&A, 213, 245

van den Oord, G. H. J., Schrijver, C. J., Camphens, M., Mewe, R., \& Kaastra, J. S. 1997, A\&A, 326, 1090

Ventura, R., Maggio, A., \& Peres, G. 1998, A\&A, 334, 188

Ventura, R., Peres, G., Pagano, I., \& Rodono, M. 1995, A\&A, 303, 509

Vilhu, O., Caillault, J. P., \& Heise, J. 1988, ApJ, 330, 922

White, N. E., Parmar, A. N., Sweeney, M. A., \& Culhane, J. L. 1987, MNRAS, 227,545

White, N. E., Shafer, R. A., Parmar, A. N., Horne, K., \& Culhane, J. L. 1990, ApJ, 350, 776

White, S. M., Jackson, P. D., \& Kundu, M. R. 1989, ApJS, 71, 895

White, S. M., Lim, J., \& Kundu, M. R. 1994, ApJ, 422, 293

Zhu, W., \& Liu, X. 1998, Chinese Astron. Astrophys., 22, 319 